\title{
LLNL/JNC Repository Collaboration Interim Progress Report
}

\author{
W. G. Halsey, W. L. Bourcier, C. E. A. Palmer, \\ T. J. Wolery, J. Gansemer, A. M. Wijesinghe, \\ R. G. Couch, K. H. Sinz, and R. B. Stout
}

July 1, 1999

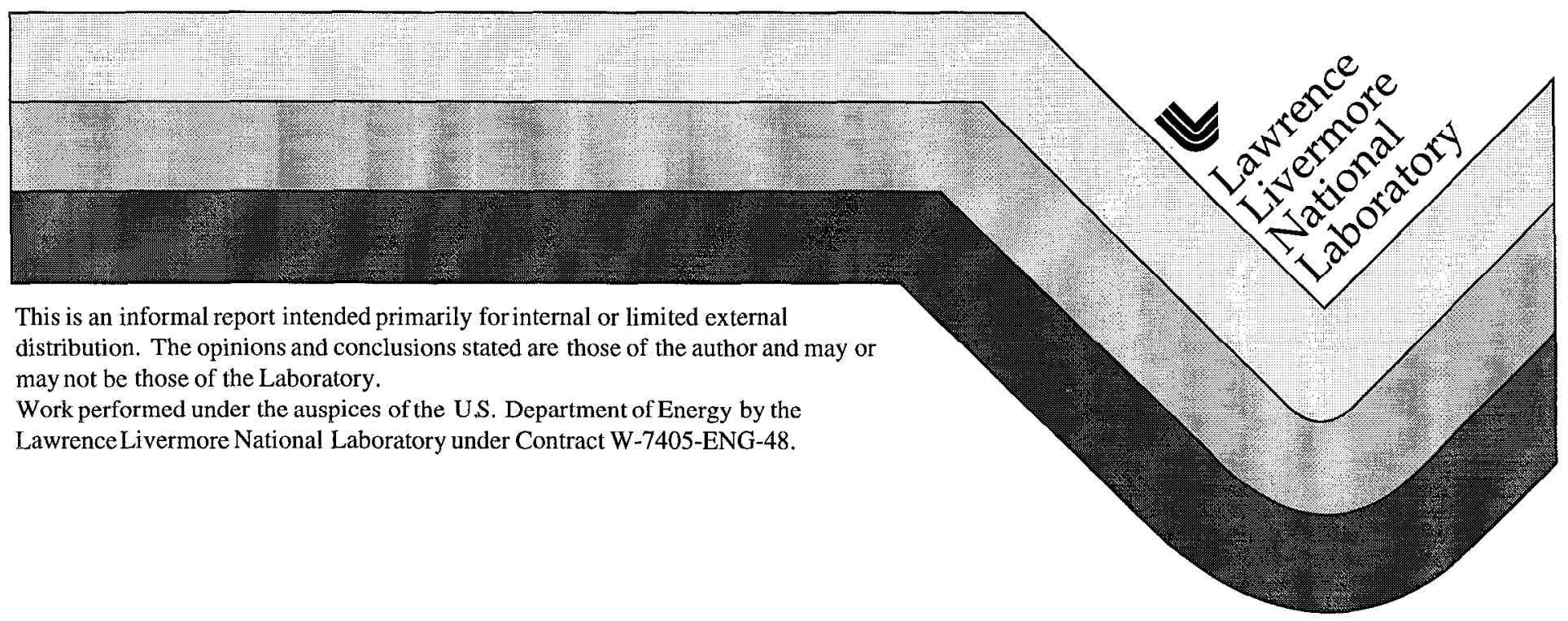




\section{DISCLAIMER}

This document was prepared as an account of work sponsored by an agency of the United States Government. Neither the United States Government nor the University of California nor any of their employees, makes any warranty, express or implied, or assumes any legal liability or responsibility for the accuracy, completeness, or usefulness of any information, apparatus, product, or process disclosed, or represents that its use would not infringe privately owned rights. Reference herein to any specific commercial product, process, or service by trade name, trademark, manufacturer, or otherwise, does not necessarily constitute or imply its endorsement, recommendation, or favoring by the United States Government or the University of California. The views and opinions of authors expressed herein do not necessarily state or reflect those of the United States Government or the University of California and shall not be used for advertising or product endorsement purposes.

This report has been reproduced directly from the best available copy.

Available to DOE and DOE contractors from the Office of Scientific and Technical Information

P.O. Box 62, Oak Ridge, TN 37831

Prices available from (423) 576-8401

Available to the public from the

National Technical Information Service

U.S. Department of Commerce

5285 Port Royal Rd.,

Springfield, VA 22161 


\section{LLNL/JNC Repository Collaboration Interim Progress Report}

July 1, 1999

Prepared for the U.S. Department of Energy under the Japan Nuclear Cycle Development Insitute (JNC) WFO Contract

Nuclear Waste Management Program Lawrence Livermore National Laboratory Livermore, CA 94551. 


\section{Contents}

Acknowledgments Acknowledgments: 1

Contents. Contents: 1

List of Figures Contents: 3

List of Tables Contents: 4

Introduction. Introduction: 1

Activity 1: Chemical Modeling of EBS Materials Interactions. Activity 1:1

Task 1.1: Chemical Modeling of Iron Effects on Borosilicate Glass Durability.... Activity 1:1

1. Introduction Activity $1: 1$

2. Experimental Data on the Effects of Dissolved Iron Activity $1: 2$

3. Experimental Measurements of the Effects of Dissolved Ferric Iron on Borosilicate Glass Dissolution Activity $1: 3$

4. Modeling the Dissolution Process. Activity $1: 4$

5. Conclusions Activity $1: 5$

6. References Activity $1: 6$

7. Tables Activity $1: 8$

Task 1.2: Changes in Overpack and Bentonite Properties Due to Metal, Bentonite and Water Interactions. Activity $1: 14$

Activity 2: Chemical Modeling of EBS Materials Interactions Activity 2:1

Task 2.1: Set up EQ3/6 to Run with the Pitzer-based PNC Thermodynamic

Database. Activity 2:1

Task 2.2: Provide Expert Consultation on Thermodynamic Database Activity $2: 1$

Task 2.3: Provide Analysis of Likely Solubility Controls on Selenium Activity $2: 1$

Activity 3: Engineered Barrier Performance Assessment of Unsaturated, Oxidizing Transient Activity $3: 1$

Task 3.1: Apply YMIM to PNC Transient EBS Performance. Activity $3: 1$

Task 3.2: Demonstrate Methods for Modeling the Return to Reducing Conditions Activity $3: 1$

Task 3.3: Evaluate the Potential for Stress Corrosion Cracking in PNC Waste Packages Activity 3:1

Activity 4: Coupled Displacement and Degradation Analysis of a Carbon Steel Overpack Embedded in Bentonite. Activity $4: 1$

Task 4.1 \& Task 4.2: Demonstration of NIKE-2D/ALE-3D Mesh Adaptation Capability and Demonstration of NIKE-2D/ALE-3D Code Capability to Compute Realistic Repository Problems. Activity $4: 1$

Executive Summary. Activity 4:1 
1. Introduction

Activity $4: 4$

1.1. Background..... Activity $4: 4$

1.2. Objective \& Approach Activity $4: 6$

2. Model Formulation

2.1. Theoretical Model Formulation Activity $4: 9$

2.1.1. Governing Field Equations Activity $4: 9$

2.1.2. Hydro-Elasto-Plastic Model for Clay Deformation, Swelling and Softening. Activity 4:9

Activity 4:14

2.1.3. Approximate Model for ALE-3D Code Computations

2.2. ALE-3D Code Selection and Application

2.2.1. Selection of the ALE-3D Code

2.2.2. Application of the ALE-3D Code

Activity $4: 18$ Activity $4: 21$ Activity $4: 21$ Activity 4:22

3. Computational Results

3.1. Problem 1: Clay Extrusion into Fracture Due to

Tunnel Roof Caving-In

Activity 4:26

3.1.1. Assumptions \& Input Data Activity $4: 26$

3.1.2. Computational Results. Activity $4: 26$ Activity $4: 28$

3.2. Problem 2: Clay Extrusion into Fracture Due to Clay Swelling and Softening.

3.2.1. Assumptions \& Input Data

Activity $4: 38$

3.2.2. Computational Results Activity $4: 38$ Activity $4: 40$

3.3. Problem 3: Overpack Displacement Due to Clay Swelling and Softening

3.3.1. Assumptions \& Input Data

3.3.2. Computational Results. Activity 4:44 Activity $4: 44$ Activity $4: 44$

4. Conclusions \& Recommendations

4.1. Conclusions Activity $4: 49$

4.1.1. Model Applicability.

4.1.2. Computational Methodology

4.2. Recommendations.

4.2.1. Model Fomulation \& Code Enhancements

4.2.2. Computations

4.2.3. Supporting Experiments Activity $4: 49$ Activity $4: 49$ Activity $4: 50$ Activity 4:51 Activity $4: 51$ Activity $4: 51$ Activity 4:52

5. References Activity $4: 53$

Task 4.3: Implementation and Verification of the Cam Clay Model in NIKE-2D/ALE-3D Code Activity $4: 54$ Task 4.4: Estimation of the Timing and Spatial Distribution of Rewetting Activity 4:54 
Summary, Conclusions \& Recommendations for Future Work Summary: 1

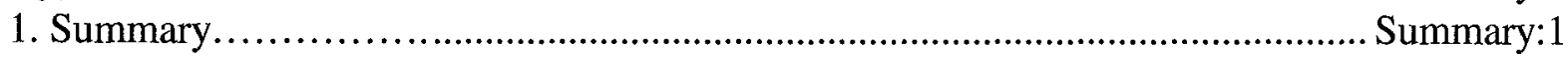

2. Conclusions \& Recommendations for Future Work..................................... Summary:3

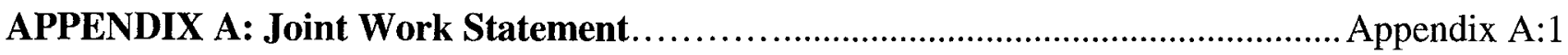




\section{List of Figures}

Figure 1

Figure 2.

Figure 3.

Figure 4.

Figure 1.1-1

Figure 1.1-2

Figure 3.1-1

Figure 3.1-2

Figure 3.1-3

Figure 3.1-4

Figure 3.1-5

Figure 3.1-6

Figure 3.1-7

Figure 3.1-8

Figure 3.1-9

Figure 3.2-1

Figure 3.2-2
Types of metal complexes on oxide surfaces (Hayes, 1987).

Activity 1:10

Two proposed mechanisms by which dissolved iron affects glass dissolution rate: (a) $\mathrm{Mg}$ in solution reacts with $\mathrm{Si}$ dissolving from glass to cause precipitation of $\mathrm{Mg}$-armoring silicate surface layer;

(b) Iron in solution reacts with Si dissolving from glass to form colloidal Fe-silicates. Activity 1:11

Calculated solubility of ferric hydroxide as a function of $\mathrm{pH}$ at

$70^{\circ} \mathrm{C}$ in dilute solution (bold line). Gray dots show nominal concentrations of dissolved iron in SPFT tests. Stability regions of ferric hydroxide species also shown.

Activity $1: 12$

Normalized loss rate (dissolution rates) for SRL-202 analog glass in $\mathrm{pH}$ buffer with and without aqueous Fe present at slightly below $\mathrm{Fe}(\mathrm{OH})_{3}$ saturation. There appears to be little effect of the dissolved ferric iron on glass dissolution rates.

Activity $1: 13$

The Engineered Barrier System Concept Activity $4: 5$

Mechanisms for Overpack Migration Activity $4: 7$

Physical Configuration for Problem 1 Activity $4: 27$

Initial finite element computational mesh at entrance to fracture (Case1 and Case-2) Activity $4: 30$

Mesh plot showing the clay interface location in fracture at 40,000 secs; Case-1: Frictionless walls with $40 \mathrm{MPa}$ applied stress. Activity $4: 31$

Mesh plot showing the clay interface location in fracture at 60,000 secs; Case-1: Frictionless walls with $40 \mathrm{MPa}$ applied stress. Activity $4: 32$ Contour plot of average normal stress disctribution in clay at 60,000 secs showing clay interface location in fracture;

Case-1: Frictionless walls and $40 \mathrm{MPa}$ applied external stress ......Activity 4:33 Mesh plot and countour plot of average normal stress distribution in clay at $7.0 \times 10^{8}$ secs showing clay interface location in fracture; Case-2: Frictional walls and $7 \mathrm{MPa}$ applied external stress .... Activity $4: 34$ Contour plot of average normal stress distribution (4 to $7 \mathrm{MPa}$ ) in clay at $7.0 \times 10^{8}$ secs showing clay interface location in fracture; Case-2: Frictional walls and $7 \mathrm{MPa}$ applied external stress Activity $4: 35$ Contour plot of plastic strain distribution $(0$ to 0.05$)$ in clay at $7.0 \times 10^{8}$ secs showing clay interface location in fracture; Case-2: Frictional walls and $7 \mathrm{MPa}$ applied external stress Activity $4: 36$ Clay interface location in fracture as a function of time; Case-1: Frictionless walls and $40 \mathrm{MPa}$ applied external stress .......Activity 4:37 The Physical Configuration for Problem 2 Activity 4:39 Contour plot of yield strength distribution (1 to $1.35 \mathrm{MPa}$ ) in clay at $4.0 \times 10^{8}$ secs. Activity $4: 41$ 
Figure 3.2-3 Contour plot of plastic strain distribution ( 0 to 0.05$)$ in clay at $4.0 \times 10^{8}$

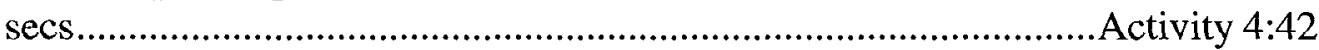

Figure 3.2-4 Contour plot of flow velocity magnitude distribution

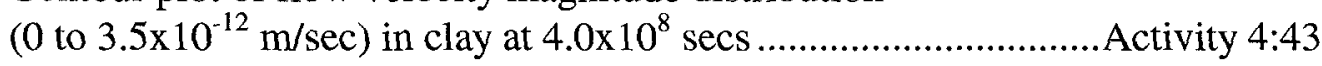

Figure 3.3-1 The Physical Configuration for Problem 3 .....................................Activity 4:45

Figure 3.3-2 Finite-element mesh for computing overpack displacement ...........Activity 4:46 Figure 3.3-3 Clay deformation velocity vector distribution (0 to $9.8 \times 10^{-8} \mathrm{~m} / \mathrm{sec}$ ) at $9.1 \times 10^{8} \mathrm{secs}$ Activity $4: 47$

Figure 3.3-4 Downward displacement of overpack as a function of time............Activity 4:48 


\section{List of Tables}

Table 1.

Table 2.

Table 3.

Table 3.1-1

Table 3.2-1
Effects of Dissolved Ions on Glass Dissolution Rate..............Activity 1:8 Composition of solutions used in flow-through dissolution tests....Activity 1:9 Compositions in mole percent oxide and cation mole percent of S-202 glass (a simple analog of SRL-202 glass).... Activity 1: 9 Problem 1: Input Model Parameter Data.... Problems 2 \& 3: Input Model Parameter Data Activity $4: 26$ Activity $4: 38$ 


\title{
Activity 1. Chemical Modeling of EBS Materials Interactions
}

\author{
Task 1.1: Chemical Modeling of Iron Effects on Borosilicate Glass \\ Durability By William Bourcier, LLNL
}

\section{Introduction}

Iron is likely to be present as part of the engineered barrier system in a high level nuclear waste repository. Corrosion of iron-containing metals will produce dissolved iron in solution, either as ferric $\left(\mathrm{Fe}^{+3}\right)$ or ferrous $\left(\mathrm{Fe}^{+2}\right)$ iron. The presence of dissolved iron may have an impact on the durability of the borosilicate glass waste form. It is therefore important to understand the effects of dissolved iron on glass dissolution in order to provide guidance for the selection of metal barriers and to optimize chemical conditions in the waste package environment.

Dissolved ions present in solution are known to affect glass dissolution rates. For example, $\mathrm{Mg}$ and $\mathrm{Zn}$ are known to decrease glass dissolution rates (Barkatt et al., 1989), (Tait and Jensen, 1982) while dissolved iron is known, under some conditions, to increase it (McVay and Buckwalter, 1983). Current glass dissolution models all account for the effect of dissolved silica on glass dissolution, but currently do not account for the effects of other ions such as iron. Although silica effects are important, and in most cases dominate over the effects of other ions, this is not always the case, and it is necessary to provide for these other ions if the model is to be generally applicable. This is especially important in repositories where the effects of species produced from corrosion of other repository materials, such as metals and cements, are available to interact with the dissolving waste forms.

Three types of mechanisms have been proposed to account for the effects of dissolved species on glass alteration rates. The first is that ions sorb onto the dissolving glass surface and affect the strength of the Si-O bonds at the glass surface (Fig. 1). It is the hydrolysis of these bonds which controls the overall rate of glass dissolution and radionuclide release. Sorption of metals onto oxygen ions at the solution-glass interface change the polarization of the Si-O bond, and therefore will affect its overall resistance to hydrolysis. This effect is particularly significant for long-term performance, where the rate at "near saturation" conditions is likely to be strongly affected by the nature of the glass-solution interface, and therefore the types and concentrations of sorbed species and net surface charge. Some attempts have been made to understand and model deviations in dissolution behavior believed to be due to surface complex formation (Lee and Clark, 1986), (Bart et al., 1987), (Grambow et al., 1987), (Andriambololona et al., 1992).

The second mechanism is the reaction of dissolved species with the glass surface to form a protective layer. The protective layer armors the glass surface and reduces the rate of further attack (Fig. 2a). The overall dissolution process then becomes rate-limited by 
transport through the protective layer. It is believed that magnesium affects glass dissolution through this process (Barkatt et al., 1989). The $\mathrm{Mg}$ combines with silica dissolving from the glass to form a protective layer of a hydrous magnesium silicate phase.

The third mechanism involves the formation of colloids by reaction of solution species with species dissolving from the glass (Fig 2b), or the formation of a large surface area of precipitate onto which species dissolving from the glass are sorbed. For example, dissolved iron reacts with silica from the dissolving glass to form iron-silica colloids. The silica-containing colloids act as a sink for silica and maintain a low dissolved silica concentration in solution. This effectively reduces the glass saturation state and therefore causes the glass dissolution rate to remain high. It has been observed experimentally that glass dissolution rates slow dramatically as dissolved silica concentrations increase (Grambow, 1987). Note that these colloids will also tend to sorb actinide species, and thus pose a potential migration pathway for otherwise insoluble actinide species.

The effect of colloids on dissolution rate can be quantified by examining the simplified rate expression commonly used to model glass dissolution is (Bourcier, 1994):

$$
R=A k\left(1-\frac{Q}{K}\right)
$$

where,

$\mathrm{R}=$ alteration rate of glass ( $\mathrm{g} / \mathrm{day})$,

$A=$ surface area of reactive glass $\left(\mathrm{m}^{2}\right)$,

$\mathrm{k}=$ glass surface alteration rate constant $\left(\mathrm{g} / \mathrm{m}^{2} /\right.$ day $)$,

$\mathrm{Q}=$ concentration of dissolved silica ( $\mathrm{mol} / \mathrm{L}$ water),

$\mathrm{K}=\mathrm{a}$ quasi-thermodynamic solubility constant for borosilicate glass.

When silica concentration in solution is reduced due to colloid formation, the value of $Q$ decreases, so that the term $(1-\mathrm{Q} / \mathrm{K})$ increases and the rate of dissolution becomes larger.

\section{Experimental Data on the Effects of Dissolved Iron}

Qualitative data on the effects of several dissolved metals on glass durability are listed in Table 1. This table summarizes a broad variety of data from experiments that in many cases are difficult to interpret due to lack of supporting data. Many of the studies for example do not report $\mathrm{pH}$. In some cases the data conflict; the same dissolved species may cause the dissolution rate to increase in one type of test and decrease in another. In other cases the glass dissolution rate may change with time. An aqueous component that first decreases glass reaction rate may enhance it later on (i.e. the case of lead reported by Zwicky et al. 1992). Another complication is that species may only have an effect if it is at a sufficiently high concentration to cause precipitation of an armoring surface solid, as is apparently the case for magnesium. At low dissolved $\mathrm{Mg}$ concentration, $\mathrm{Mg}$ has no noticeable effect (Bourcier et al., 1992) but at higher concentrations Mg decreases the 
glass reaction rate. Table 1 shows that there is a lack of data for many metals likely to be present in the repository (i.e. alloying metal in stainless steels such as $\mathrm{Cr}, \mathrm{Mn}, \mathrm{Ni}$, Mo etc.).

Although this qualitative information is available, it alone is not sufficient for incorporation of these effects into models of glass dissolution. Experiments are needed which both identify the mechanisms through which dissolved species affect dissolution rates, and provide the parameters needed to quantify these effects in the glass dissolution models. Explicit provision for surface interactions will be especially critical in order to account for coupled effects of glass with other repository materials in performance assessment calculations.

\section{Experimental Measurements of the Effect of Dissolved Ferric Iron on Borosilicate Glass Dissolution}

Data exist on the effect of dissolved ferric iron on borosilicate glass dissolution rates ((Stout and Leider, 1998). In these experiment, glass powder was dissolved in single-path flow through cells (SPFT) using pH buffers of ortho-phthalic acid, borate, and $\mathrm{KOH}-\mathrm{KCl}$ at ionic strengths of 0.005 molal (see Table 2). Powdered glasses (surface area $=450 \mathrm{~cm}^{2} / \mathrm{g}$ ) in $3 \mathrm{ml}$ cells were reacted with buffer solutions at flow rates of $50-100$ $\mathrm{ml} /$ day. Half of the $\mathrm{pH}$ buffers were doped with $\mathrm{Fe}^{3+}$ (added as $\mathrm{FeCl}_{3}$ ) with their iron concentrations all nominally slightly undersaturated with respect to ferric hydroxide, and slightly oversaturated with respect to goethite $\left(\mathrm{FeO}(\mathrm{OH})_{(\mathrm{s})}\right)$ (see Fig. 3). This range of iron concentrations is reasonable for an oxidizing repository with actively corroding ironcontaining metals present (Fig. 3). This is likely to be the maximum dissolved iron concentration in repository waters because ferric hydroxide readily precipitates from supersaturated solutions, but ages with time to goethite. Control experiments with no dissolved iron were run for comparison.

A 5-component analog of the SRL-202 glass composition was used in the test rather than SRL-202 glass (composition given in Table 4). The analog was prepared by adjusting the mole fraction of each component in the analog glass to be equal to the sum of the mole fractions of the components in the actual glass which were judged to occupy similar structural sites in the glass. This determination was based on crystal chemical principles including mainly ion size and radius. For example, the sodium content of the S-202 glass is determined by adding the molar concentrations of all the alkalis in the SRI-202 glass. Similar calculations were performed for $\mathrm{Ca}, \mathrm{Al}$, and $\mathrm{Si}$ in the analog glass. The mole fraction of $B$ was kept equal to that of the waste glass. The simple glasses avoid problems in data interpretation due to redox reactions during dissolution and precipitation of insoluble secondary phases in real waste glasses.

The experimental data from these tests typically show steady state dissolution rates after a few days. No change in steady-state dissolution rates were found when flow rates were doubled, indicating the systems behaved as continuously stirred reactors in that the dissolution rates were not rate-limited by transport away from the glass reaction surface. 
The effects of doping the buffers with Fe on the glass dissolution rate can be seen in Fig. 4. which shows the release rate of silica from the glass as a function of $\mathrm{pH}$ for both undoped and doped buffers. Apparently, dissolved iron has little effect on glass dissolution rates over the $\mathrm{pH}$ range and iron concentration range tested. The data points for the iron-doped and undoped buffers lie essentially on top of each other and their differences in all cases are less than the precision of the experimental method. These results are consistent with several previous studies of the effect of iron on borosilicate glass dissolution where borosilicate glass was leached in the presence of various metal phases including 304L stainless steel, the pour canister material (Bates et al., 1988; Bibler and Jantzen, 1987; Burns et al., 1986). In all of these studies, the iron had little or no effect on the glass dissolution rate.

However, other studies have noted an enhancement of glass dissolution rates presumably due to the presence of iron-containing materials in the system [(McVay and Pederson, 1981); (Bart et al., 1987); (Inagaki et al., 1996)]. It is concluded in each of these studies that it is the sorption of silica onto iron colloids, or the sorption of silica onto iron-containing solids, that causes the glass to dissolve faster by lowering the silica concentration in solution. The system is therefore farther from silica saturation and the glass dissolves faster under those conditions. Presumably, these later experiments contained iron solids with a higher surface area than tests where no iron effect was noticed. In some cases, the iron-containing material was much more reactive than $304 \mathrm{~L}$ stainless steel i.e. (McVay and Pederson, 1981) used ductile iron, which corrodes much more rapidly and evidently gave rise to iron colloids.

We conclude that the presence of iron in the waste repository can have a significant negative impact on borosilicate glass performance only if it either leads to the development of colloids which sorb silica, or presents a large amount of surface area for silica sorption. In both cases, the amount of colloids or surface area must be of sufficient quantity to significantly decrease the concentration of dissolved silica due to sorption. The presence of dissolved iron alone apparently has little effect on the glass dissolution rate. Note that no colloids were present in the SPFT experiments described here.

\section{Modeling the Dissolution Process}

Three potential methods through which dissolved iron can affect glass dissolution were listed above. Each can be included in geochemical models of the glass dissolution process given the necessary code development and data base enhancements.

\section{Case 1, Surface Sorption of Iron:}

In this case, sorption of dissolved iron on the glass surface takes place, which affects the strength of the Si-O bond at the glass-water interface. The method commonly used to model this process is to express the rate coefficient ( $\mathrm{k}$ in Eqn. 1) in terms of the population of surface sites which includes those at which an Fe ion is present, protonated sites (where an $\mathrm{H}^{+}$is present) and 
bare sites. Each site has its own rate constant. The overall rate is a weighted average of all types of sites. Other workers have used this approach to successfully model the effects of $\mathrm{Na}^{+}$on quartz dissolution (Dove and Elston, 1992), and $\mathrm{pH}$ on kaolinite dissolution (Carroll-Webb and Walther, 1988).

For this approach, experimental data are needed which provide the dissolution rate as a function of dissolved iron concentration (providing for both $\mathrm{Fe}^{2+}$ and $\mathrm{Fe}^{3+}$ as separate species), and the density of iron sorption sites on the surface obtained from either surface titrations or spectroscopic data on surface site densities (Sahai et al., 1999). Care must be taken to account for any iron precipitates which may armor the surface and exclude that effect from the sorption term.

\section{Case 2, Formation of a protective layer:}

If a protective layer forms on the glass surface, the overall reaction becomes transportlimited and the appropriate rate law must be used. It is commonly assumed that bulk diffusion of species through the layer, or surface diffusion along grain boundaries in the layer, is the ratelimiting transport mechanism (Cussler, 1984). This scenario is problematic in that the protective layers may coarsen with time and spall from the surface, opening it up to further dissolution. This is commonly observed for borosilicate glasses. Rarely do protective layers form which remain intact over time. Although the experiments in Mg-rich solutions have shown this behavior, it is not known whether these short-term results of a few weeks can safely be extrapolated to repository-type time frames. No protective layer formation has yet been observed in glass durability tests with dissolved iron.

\section{Case 3, Formation of Colloids:}

In this case, dissolved species from the glass (in particular $\mathrm{Si}$ ) react with dissolved species (Fe) to form colloids, which lower the saturation state of the glass. This mechanism can be incorporated into existing geochemical modeling codes. A fictitious solid can be entered into the database having the stoichiometry of the colloid. An appropriate solubility product can be regressed from experimental data so that as the glass dissolves, precipitation of the colloid maintains Si and Fe levels to those observed in the dissolution tests. This approach can then be used to predict long-term effects as the colloids flocculate and transform into more stable phases. The precipitation rates of the stable phases would control the overall change in solution composition and therefore glass dissolution rate.

\section{Conclusions}

It is clear that it is not yet possible to quantitatively model the effects of dissolved species such as iron on glass dissolution rates. If the dissolution rate is controlled by the presence of sorbed iron, additional experimental work, and some code development work, are needed. The basic experimental work needed is a matrix of glass dissolution rate measurements in iron-containing solutions over a range of $\mathrm{pH}$ values and temperatures which can be used to quantify the effect of iron on the rate coefficient as a function of $\mathrm{pH}$ and temperature. These 
measurements should be combined with surface titrations or spectroscopic data to quantify the sorption density of iron on the glass surface.

If protective layers are rate limiting, and given the problem of predicting how long they remain protective, it may be prudent to make the conservative assumption that the layer is not protective and that the overall rate is limited by solution chemistry in equilibrium with a stable assemblage of alteration minerals.

Finally, in order to model the formation of colloids and their effects on glass dissolution, we need improved data on the compositions of colloids and their stability over time. Again, a conservative model where solution concentrations are assumed to be controlled by some stable set of alteration minerals (which will always have lower solubilities than colloids) provides an upper limit to the rate of glass dissolution.

\section{References}

Andriambololona Z., Godon N., and Vernaz E. (1992) R7T7 glass alteration in the presence of mortar: Effect of the cement grade. Mat. Res. Soc. Symp. Proc. 257:151-158.

Barkatt A., Saad E. E., Adiga R., Sousanpour W., Barkatt A., and Adel-Hadadi M. A. (1989) Leaching of natural and nuclear waste glasses in sea water. Appl. Geochem. 4:593-603.

Bart G., Zwicky H. U., Aerne E. T., Graber T., Z'Berg D., and Tokiwai M. (1987) Borosilicate glass corrosion in the presence of steel corrosion products. Mat. Res. Soc. Symp. Proc. 84:459-470.

Bates J. K., Ebert W. L., Fisher D. F., and Gerding T. J. (1988) The reaction of reference commercial waste glasses during gamma irradiation in a saturated tuff environment. $J$. Mat. Res. Soc. 3:576-597.

Bibler N. E. and Jantzen C. M. (1987) Materials interactions relating to long-term geologic disposal of nuclear waste glass. Mat. Res. Soc. Symp. Proc. 84:47-66.

Bourcier W. L., (1994) Critical review of glass performance modeling, Argonne National Laboratory, Report Number ANL-94/17; 1994.

Bourcier W. L., Weed H. C., Nguyen S. N., Nielsen J. K., Morgan L., Newton L., and Knauss K. G. (1992) Solution compositional effects on dissolution kinetics of borosilicate glass. Seventh Annual Water-Rock Conference, 81-84.

Burns D. B., Upton B. H., and Wicks G. G. (1986) Interactions of SRP waste glass with potential overpack materials. J. Non-Cryst. Solids 84:258-267.

Carroll-Webb S. and Walther J. V. (1988) A surface complex reaction model for the pHdependence of corundum and kaolinite dissolution rates. Geochim. Cosmochim. Acta 52:2609-2623.

Cussler E. L. (1984) Diffusion: Mass Transfer in Fluid Systems. Cambridge University Press. $525 \mathrm{p}$.

Dove P. M. and Elston S. F. (1992) Dissolution kinetics of quartz in sodium chloride solutions: Analysis of existing data and a rate model for 25 C. Geochim. Cosmochim. Acta 56:41474156.

Grambow B., (1987) Nuclear waste glass dissolution: Mechanism, model and application., JSS Project, Swedish Nuclear Fuel and Waste Management Co., Report Number 87-02; 114 p.; 
Grambow B., Zwicky H. U., Bart G., Björner I. K., and Werme L. O. (1987) Modeling the effect of iron corrosion products on nuclear glass performance. Mat. Res. Soc. Symp. Proc. 84:471-481.

Inagaki Y., Ogata A., Furuya H., Idemitsu K., Banba T., and Maeda T. (1996) Effects of redox condition on watse glass corrosion in the presence of magnetite. Mat. Res. Soc. Symp. Proc. 412:257-264.

Lee C. T. and Clark D. E. (1986) Effects of solution cations on waste glass leaching. In Advances in Ceramics, Vol. 20. American Ceramic Society.

McVay G. L. and Buckwalter C. Q. (1983) Effect of iron on waste-glass leaching. J. Amer. Cer. Soc. 66(3):170-174.

McVay G. L. and Pederson L. R. (1981) Effect of gamma radiation on glass leaching. J. Amer. Cer. Soc. 64:154-158.

Sahai N., O'Day P., Carroll S., and Roberts S. (1999) Spectroscopic and chemical characterization of strontium sorption at goethite and kaolinite surfaccs. J. Colloid Interface Sci. in press.

Stout R. B. and Leider H. R., (1998) Waste Froms Characteristics Report, Lawrence Livermore National Laboratory, Report Number UCRL-ID-132375; 656 p.; December 1998.

Tait J. C. and Jensen C. D. (1982) The effect of $\mathrm{Zn}$ (II) ion adsorption on the durability of sodium borosilicate glasses. J. Non-Cryst. Solids 49:363-377. 


\section{Tables}

\section{Table 1. Effects of Dissolved Ions on Glass Dissolution Rate.}

\begin{tabular}{|c|c|c|c|c|}
\hline Metal & Low pH & Near-neutral pH & High pH & Reference \\
\hline $\mathrm{B}$ & none & none & unknown & 1 \\
\hline $\mathrm{Mg}$ & none & major - & major - & $1,2,3,18$ \\
\hline $\mathrm{Ca}$ & none & none & minor - & $1,3,13$ \\
\hline $\mathrm{Si}$ & none & major - & major - & 1,12 \\
\hline $\mathrm{Al}$ & major - & minor - & none/minor + & $1,3,11,15$ \\
\hline $\mathrm{Na}$ & unknown & minor - & minor - & 3 \\
\hline $\mathrm{Zn}$ & unknown & minor + & major - & $3,11,14$ \\
\hline $\mathrm{Li}$ & unknown & unknown & minor + & 4 \\
\hline $\mathrm{Fe}$ & unknown & major/minor + & major + & $5,6,7,9,10,16,17$ \\
\hline $\mathrm{Pb}$ & unknown & major - & major - & $7,8,10,11,15$ \\
\hline $\mathrm{Cu}$ & unknown & unknown & none & 15 \\
\hline $\mathrm{Sn}$ & unknown & unknown & none & 15 \\
\hline $\mathrm{Ti}$ & unknown & unknown & none & 15 \\
\hline
\end{tabular}

Notes: minor - less than factor of 10 effect; major - greater than factor of 10 effect; + means glass dissolution rate is increased, - means rate is decreased; none - no effect observed; unknown - no data or data uninterpretable.

\section{References for Table 1 :}

(1) Bourcier et al., 1992, Proc. 7th Int. Symp. Water-Rock Interaction, p. 81-84.

(2) Barkatt et. al., 1989, Applied Geochem. 4:593-603.

(3) Lee and Clark, 1986, Adv. in Ceramics Vol 20, p. 541-550

(4) Feng and Barkatt (1987) Mat. Res. Soc Symp. Proc, Vol 84, p. 519-531.

(5) Bunker and Arnold (1983) Mat. Res. Soc Symp. Proc, Vol 15, p. 151-158

(6) McVay and Buckwalter (1983), J. Am. Cer. Soc., 66:170-174.

(7) Bart et al. (1987) Mat. Res. Soc Symp. Proc, Vol 84, p. 459-470.

(8) Lehman and Kuchinski (1985) Mat. Res. Soc Symp. Proc, Vol 44, p. 179-186

(9) Bibler and Jantzen (1987) Mat. Res. Soc Symp. Proc, Vol 84, p. 47-66

(10) Burns et al. (1986) J. Noncryst. Solids 84:258-267.

(11) Zwicky et al., (1992) Mat. Res.Soc Symp. Proc, Vol 257, p. 83-90.

(12) Lanza et al., (1988) Mat. Res.Soc Symp. Proc, Vol 112, p. 685-691.

(13) Oka et al., (1979) J. Am. Cer. Soc. 62:631-632.

(14) Tait and Jensen (1982) J. Noncryst. Solids 49:363-377.

(15) Buckwalter and Pederson (1982) J. Am. Cer. Soc., 65:431-436.

(16) Hermansson et al. (1985) Nuc. and Chem. Waste Manag. 5:315-332.

(17) Inagaki et al., (1996) Mat. Res.Soc Symp. Proc, Vol 412, p. 257-264.

(18) Sang et al., (1994) Mat. Res.Soc Symp. Proc, Vol 333, p. 519-524. 
Table 2. Composition of solutions used in flow-through dissolution tests.

\begin{tabular}{|c|c|c|}
\hline $\mathbf{p H}$ & Buffer & Iron Concentration \\
\hline 6 & 0.005 molal Ortho-phthalic acid $+\mathrm{KOH}$ & $3.3 \times 10^{-7} \mathrm{molal} \mathrm{FeCl}_{3}$ \\
\hline 8 & 0.005 molal Boric Acid $+\mathrm{KOH}$ & $7.8 \times 10^{-8} \mathrm{molal} \mathrm{FeCl}_{3}$ \\
\hline 10 & 0.005 molal Boric Acid $+\mathrm{KOH}$ & $2.6 \times 10^{-7} \mathrm{molal} \mathrm{FeCl}_{3}$ \\
\hline 12 & 0.013 molal KOH & $2.2 \times 10^{-5} \mathrm{molal} \mathrm{FeCl}_{3}$ \\
\hline
\end{tabular}

Table 3. Compositions in mole percent oxide and cation mole percent of S-202 glass (a simple analog of SRL-202 glass).

\begin{tabular}{|c|c|c|c|c|}
\hline Oxide /Cation & \multicolumn{2}{|c|}{ Mole \% Oxide } & \multicolumn{2}{c|}{ Cation Mole \% } \\
\hline & SRL-202 & S-202 & SRL-202 & S-202 \\
\hline $\mathrm{SiO}_{2}$ & 55.44 & 55.6 & 40.1 & 40.9 \\
\hline $\mathrm{Al}_{2} \mathrm{O}_{3}$ & 2.56 & 8.1 & 3.7 & 11.5 \\
\hline $\mathrm{Fe}_{2} \mathrm{O}_{3}$ & 4.86 & -- & 7.0 & - \\
\hline $\mathrm{B}_{2} \mathrm{O}_{3}$ & 7.79 & 8.0 & 11.3 & 11.3 \\
\hline $\mathrm{Na}_{2} \mathrm{O}$ & 9.79 & 22.7 & 14.2 & 32.3 \\
\hline $\mathrm{Li}_{2} \mathrm{O}$ & 9.63 & -- & 13.9 & - \\
\hline $\mathrm{CaO}$ & 1.46 & 5.7 & 1.2 & 4.1 \\
\hline $\mathrm{MgO}$ & 2.23 & -- & 1.6 & - \\
\hline $\mathrm{MnO}$ & 1.10 & --- & 0.6 & -- \\
\hline $\mathrm{SrO}$ & 0.02 & --- & 0.01 & -- \\
\hline $\mathrm{BaO}$ & 0.10 & --- & 0.07 & -- \\
\hline $\mathrm{NiO}$ & 0.75 & --- & 0.5 & -- \\
\hline $\mathrm{U}_{3} \mathrm{O}_{8}$ & 0.16 & --- & 0.3 & - \\
\hline
\end{tabular}




\section{Figures}

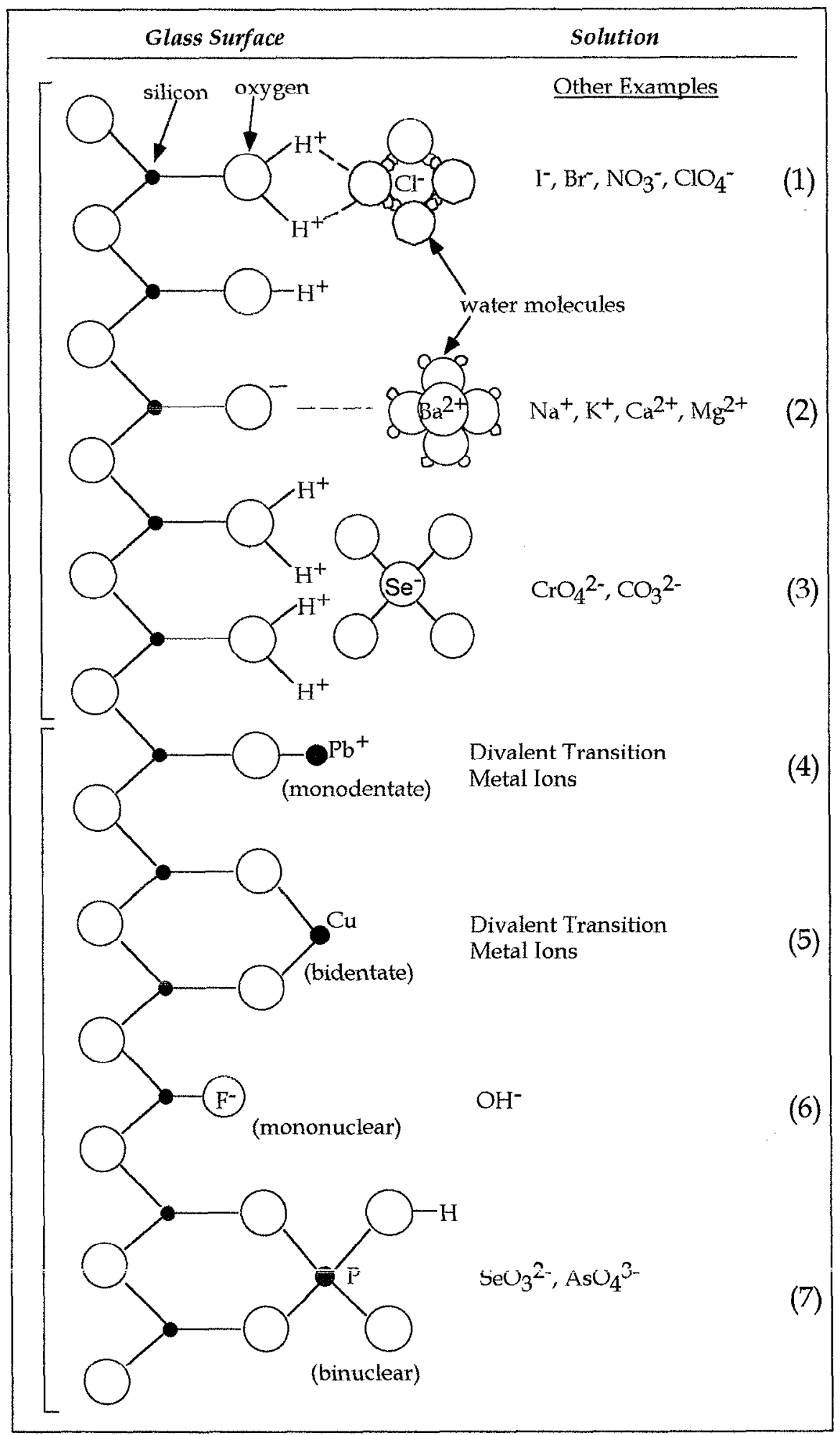

Figure 1. Types of metal complexes on oxide surfaces (Hayes, 1987). 

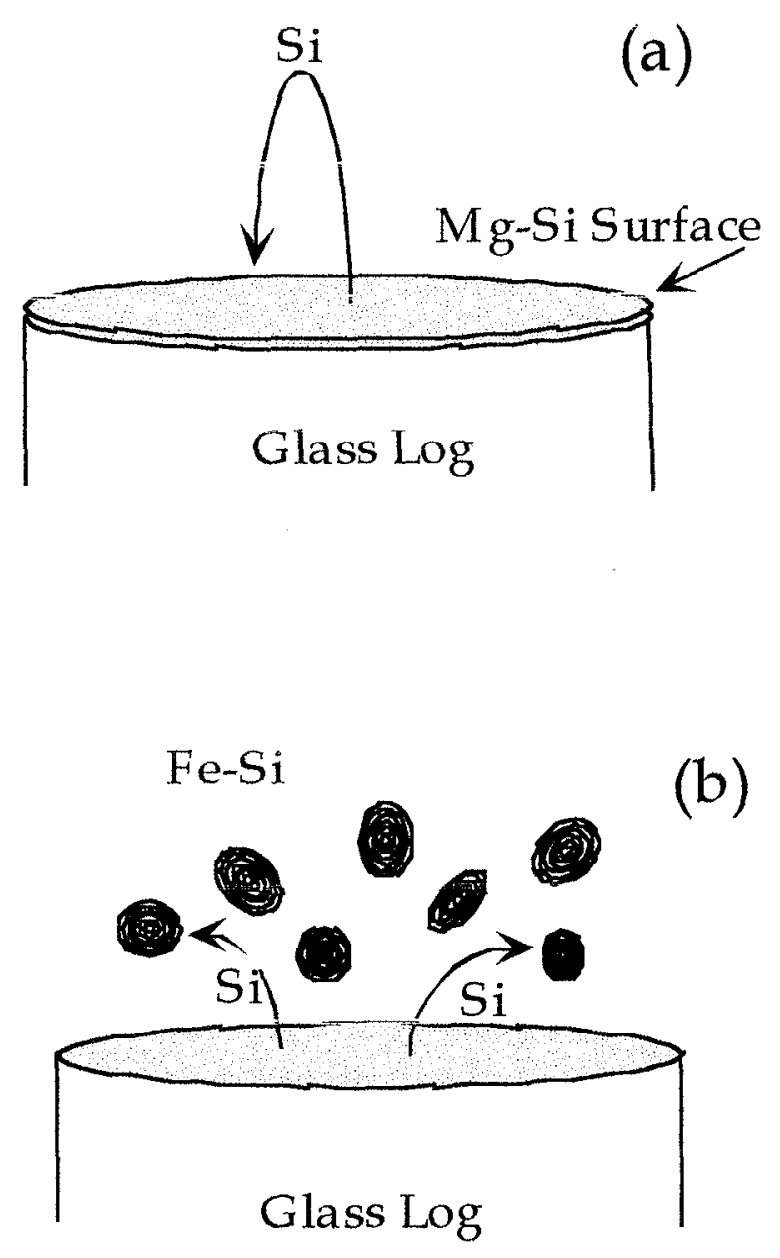

Figure 2. Two proposed mechanisms by which dissolved iron affects glass dissolution rate: (a) $\mathrm{Mg}$ in solution reacts with Si dissolving from glass to cause precipitation of $\mathrm{Mg}$-armoring silicate surface layer; (b) Iron in solution reacts with Si dissolving from glass to form colloidal Fe-silicates. 


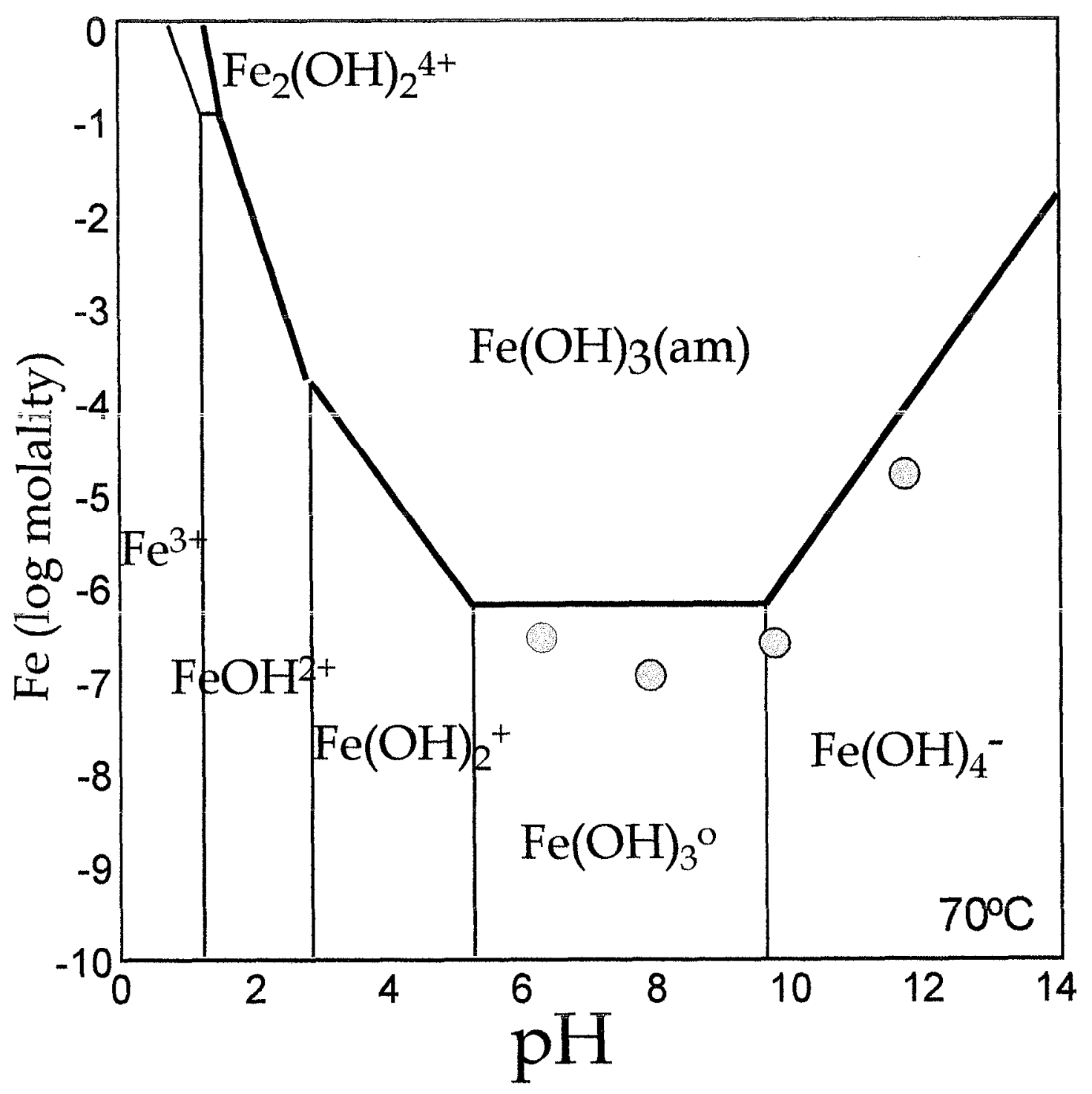

Figure 3. Calculated solubility of ferric hydroxide as a function of pH at $70^{\circ} \mathrm{C}$ in dilute solution (bold line). Gray dots show nominal concentrations of dissolved iron in SPFT tests. Stability regions of ferric hydroxide species also shown. 


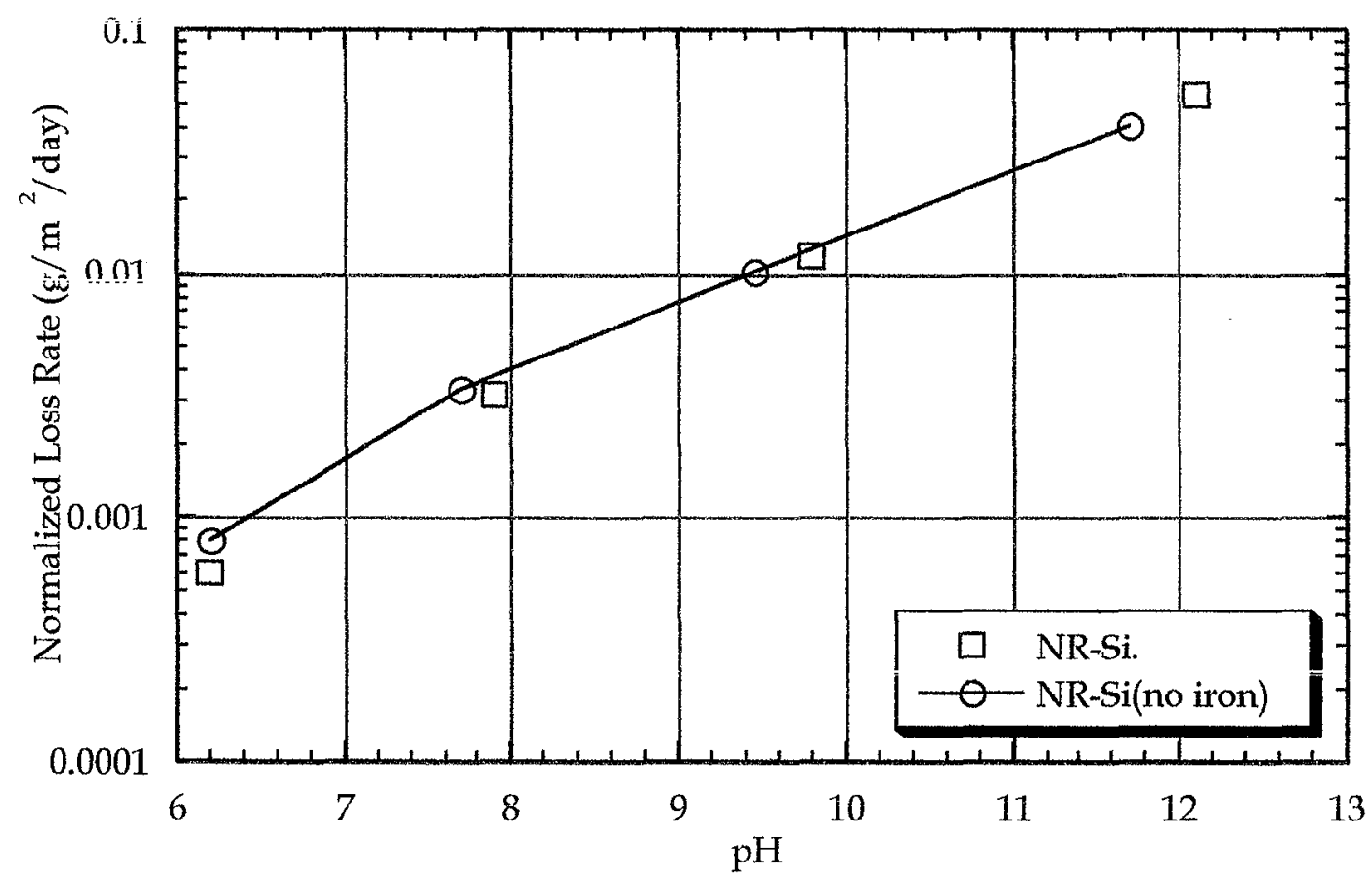

Figure 4. Normalized loss rate (dissolution rates) for SRL-202 analog glass in $\mathrm{pH}$ buffer with and without aqueous Fe present at slightly below $\mathrm{Fe}(\mathrm{OH})_{3}$ saturation. There appears to be little effect of the dissolved ferric iron on glass dissolution rates. 


\subsection{Task 1.2: Changes in Overpack and Bentonite Properties Due to} Metal, Bentonite and Water Interactions By Brian Viani, LLNL

No work was performed in this sub-Task in the current reporting period. 


\title{
Activity 2. Thermodynamic Database Validation and Comparison
}

\author{
Task 2.1: Set up EQ3/6 to Run with a Pitzer-based JNC \\ Thermodynamic Data By C.E.A Palmer and T.J. Wolery, LLNL
}

This task is modifying EQ3/6 to allow it to make calculations using an expanded thermodynamic database for JNC. This database will support the use of Pitzer's equations, including a new format in which the Pitzer coefficients are represented by temperature functions as exemplified for example in the work of Greenberg and Møller (1989, Geochimica et Cosmochimica Acta, 53, 2503-2518). This format provides a much more accurate way to make Pitzer-based calculations at elevated temperatures. New as well as currently available data (including JNC data) will be incorporated into GEMBOCHS, the LLNL relational database for thermodynamic data. GEMBOCHS will be modified to produce a JNC data file for EQ3/6. The new database will thus allow JNC investigators to make geochemical calculations using EQ3/6 (as well as other codes that use $\mathrm{EQ} 3 / 6$ data files).

GEMBOCHS development has been frozen the past several months awaiting a necesssary hardware upgrade, so very limited progress has been made to date. Also, existing JNC thermodynamic data have yet to be received. A prototype Greenberg-Møller data file has been constructed. The EQPT code (which is the data file preprocessor for EQ3/6) has been substantially upgraded to process Pitzer data. It now provides substantially better diagnostics of possible problems with such data. For example, a warning is now issued if any ternary system data are present but with missing data for a constituent binary system. This is but one of a substantial number of special conditions which are now checked by the code.

\section{Task 2.2: Provide Expert Consultation on the Thermodynamic Database By C.E.A Palmer and T.J. Wolery, LLNL}

This task will provide an LLNL expert to provide expert consultation on the thermodynamic database. The expert is expected to be Dr. Robert N. Silva. Work on this task has not yet started.

\section{Task 2.3: Provide Analysis of Likely Solubility Controls on Selenium $B y$ C.E.A Palmer and T.J. Wolery, LLNL}

Selenium is a major concern in the early stages of JNC waste form leaching. The concentration of dissolved selenium may be limited by the formation of $\mathrm{Se}_{(\mathrm{c})}$ and $\mathrm{FeSe}_{2(\mathrm{c})}$, or by substitution of $\mathrm{Se}$ for $\mathrm{S}$ in the corresponding $\mathrm{S}$ phases. This task will analyze 
experimental solubility data to be provided by JNC to see if it can be modeled assuming thermodynamic equilibrium. Work on this task has not yet started. 


\section{Activity 3. Engineered Barrier Performance of the Unsaturated, Oxidizing Transient}

\section{Task 3.1: Apply YMIM to JNC Transient EBS Performance By James Gansemer, $L L N L$}

The waste package currently under consideration by JNC is a single, thick-walled container constructed from materials similar to those under consideration by YMP. The JNC repository environment at ambient conditions will be a saturated reducing environment, however, during the transient period initiated by decay heat, oxidizing unsaturated conditions may exist in the vicinity of the waste that are similar to those expected at the YMP site. During this transient period, several waste package corrosion regimes may be active for which models have been or will be developed for use in YMP that may be applicable to the JNC design. These include: general aqueous corrosion, stress corrosion cracking, and a localized "pitting" model.

LLNL has provided a GC and localized corrosion model to YMP for use in TSPAVA. These models, with modifications resulting from further study, will be used in the JNC analysis. Corrosion testing is currently underway to provide updated general corrosion data. This data will become available during the second half of the fiscal year, and the GC model used for the JNC analysis will make use of this updated information. Early analyses have indicated that for the expected repository environment, SCC will not be an issue, however, model development in this area is anticipated to be pursued during the second half of the fiscal year and may provide some second order effects on waste package lifetime. While data developed for use by YMP may not be directly applicable due to differences in material selection and THC conditions, it is expected that this data can be extrapolated to $\mathrm{INC}$ conditions.

The inputs required by the WP degradation model are: thermal history, $\mathrm{RH}$ in the vicinity of the WP, fluid flux and composition, and oxygen availability.

\section{Task 3.2: Demonstrate Methods for Modeling the Return to Reducing Conditions By Thomas Wolery, LLNL}

No activity during current reporting period.

\section{Task 3.3: Evaluate the Potential for Stress Corrosion Cracking in JNC Waste Packages By Gregory Gdowski, LLNL}

No activity during current reporting period. 


\title{
Activity 4. Coupled Displacement and Degradation Analysis of a Carbon Steel Overpack Embedded ion Bentonite
}

\author{
Task 4.1: Demonstration of NIKE-2D/ALE-3D Mesh Adaptation \\ Capability \& Task 4.2: Demonstration of NIKE-2D/ALE-3D Code \\ Capability to Compute Realistic Repository Problems By Ananda $M$. \\ Wijesinghe (PI), Richard G. Couch, Kurt H. Sinz (PI), and Ray B. Stout, ILLNL
}

\section{EXECUTIVE SUMMARY}

This report is an account of work performed in Tasks 4.1 and 4.2 of the Lawrence Livermore National Laboratory/Japan Nuclear Corporation Repository Collaboration Project. In this report, we test and demonstrate our capability to model and compute the displacement of waste packages emplaced in clay-filled tunnels. This displacement is driven by coupled processes that involve the transport of groundwater in buffer materials, the displacement and alteration of overpack and buffer materials, and the extrusion of buffer material into rock fractures. ALE-3D is a hydro-elasto-plastic code for modeling mechanical deformation of materials coupled to heat conduction. Its arbitrary Lagrangian-Eulerian formulation, and the capability to represent frictional interfaces as slide surfaces, offers great computational flexibility for modeling problems that involve large material deformation and coupled physical processes occurring on disparate time and length scales. The ALE-3D code was selected for this test, in preference to other available codes, primarily because its arbitrary Lagrangian-Eulerian computational capability accomodates severe material deformation through automatic remeshing of the computational grid and high-order remapping of the physical variables during the remeshing process. The computational capabilities of the ALE-3D code demonstrated here may have significant implications not only for assessing problems of overpack migration, but also for assessing the impact of coupled processes that affect the long-term drainage of drift water on repository performance.

We developed a rigorous theoretical model of the coupled processes that govern water transport within a porous elasto-plastically deforming clay material subject to swelling. We made several approximations to reduce this rigorous formulation to the governing equations solved in the ALE-3D code. This permitted us to use the existing ALE-3D code to approximately model and solve the problems at hand. The most important of these approximations are:

1. The clay behaves as a porous deformable material satisfying an effective stress law for partititiong the total stress between the solid and fluid phases, 
2. The constitutive behavior of effective stress for the clay solid satisfies an associated Drucker-Prager type frictional elasto-plastic flow model extended to incorporate isotropic swelling of clay and water saturation dependent plasticity parameters,

3. The transport of water within the clay under unsaturated conditions can be described in terms of relative permeability and capillary pressure relationships,

4. The pressure of the air phase within the pores remains essentially constant.

Under these assumptions, the governing equations were reduced to a form that is analogous to the governing equations used for modeling coupled heat conduction and thermo-elasto-plasto-dynamic deformation in the ALE-3D code when water saturation is identified as the analog of temperature. We believe all of the underlying approximations to be reasonable and valid in this application. We also used the well established technique of density scaling to solve our quasi-static problems with the dynamic ALE-3D code on a time scale of years instead of the usual time scale of the order of seconds or less.

We have successfully completed sub-Task 4.1: Demonstration of NIKE-2D/ALE$3 D$ Mesh Adaptation Capability, and have demonstrated that the ALE-3D code is capable of modeling clay extrusion into fractures, by successfully computing an uncoupledprocesses problem and a coupled-processes problem. In both problems, a single vertical fracture, $1-\mathrm{mm}$ wide, was located at the center of the floor of a $0.5-\mathrm{m}$ wide $\times 0.5-\mathrm{m}$ tall rigid-walled clay-filled tunnel of infinite length. In the first uncoupled-processes problem, the driving force for clay extrusion was a uniform pressure applied to the clay at the roof of the tunnel. In the second coupled-processes problem, the driving force was derived from the swelling of the clay when wetted by water that infiltrated into the clay from the tunnel roof. The ALE-3D code computed these two problems without any difficulty in handling the extreme distortion of the clay at the entry to the fracture and the movement of a wetting front into the clay. We treated the tunnel and the fracture as rigid and used estimated material properties for the clay.

In sub-Task 4.2: Demonstration of NIKE-2D/ALE-3D Code Capability to Compute Realistic Repository Problems, a more realistic problem of the downward migration of a steel overpack emplaced in a clay-filled tunnel was modeled, withou considering the impact of clay extrusion into an underlying fracture. The migration was due to elasto-plastic flow of clay around the overpack driven by the weight of the overpack and the stress increase due to clay swelling above the overpack due to water influx at the tunnel roof. Frictional interfaces were used along the tunnel walls and the overpack surface. Displacement of the overpack varied from no settlement to some settlement as the yield strength was reduced over a practical range. The frictional shear stress along the sides of the overpack was a significant factor that retarded the downward movement of the overpack.

We have demonstrated the ability of the ALE-3D code to handle the large distortions associated with clay extrusion into fractures, the principal issue of interest to the JNC. We showed that the ALE-3D code can also compute overpack migration 
problems involving coupled processes of plastic flow and water infiltration. However, the limited scope of our effort precluded refinement of the clay material properties, and the accuracy of the results was not independently verified. This was due to limitations in the available funds, project time and material property data. As such, no conclusion on the significance of impact of clay extrusion into fractures on overpack migration can be made at this time on the basis of this limited study; that must await the results of the more detailed parametric study that will be undertaken in sub-Tasks 4.3 and 4.4. Furthermore, we believe that other elasto-plastic models, such as Cam Clay critical-state models and Sandler-DiMaggio moving cap models, should also be evaluated for use in this application to decide on the best approach. Therefore, in the next phase of this Task, we propose to implement these model enhancements, and evaluate and verify the modeling results using more appropriate material property experimental data and through comparison against other calculations or direct measurements of overpack displacement and clay extrusion into fractures. 


\section{INTRODUCTION}

\subsection{BACKGROUND}

The current concept for geologic disposal of radioactive waste in Japan is based on a multiple barrier disposal system to be sited in a stable geologic environment. This system will be designed to provide an adeqate margin of safety against the release of radioactivity to the accessible environment over a very long time period [1]. The multiple barrier system consists of the geologic barrier between the accesssible environment and the system of underground tunnels in which the waste is emplaced, and an engineered barrier system which consists of the waste packages and the sealing buffer materials that fill the emplacement tunnels. As shown in Figure 1.1-1, the engineered barrier is itself a multiple barrier system. It consists of the vitrified waste, the canister that contains this waste, the steel overpack that encloses the waste canister, and the buffer materials that fill and seal the space within the tunnel between the steel overpack and the rock mass.

The primary means by which the radioactive waste would be released in this disposal system is through contact with flowing groundwater. Over a very long period of time, groundwater is expected to permeate the buffer material; to corrode and degrade the overpack and canister materials; to contact, transform, and dissolve the vitrified radioactive waste; and to transport the radionuclides as solutes or colloidal particles to the accessible environment.

The buffer material, usually a clay or a mixture of sand and clay, that fills the space between the steel overpack and the wall of emplacement cavity, is expected to serve as a low permeability hydraulic barrier to the influx of corrosive groundwater. When the overpack is corroded by the incoming groundwater and finally breached, the buffer material is expected to serve as a chemical barrier to the transport of the mobilized radionuclides by the flowing groundwater by sorbing/reacting with the released radionuclides present in the water. Therefore, it is important to preserve unchanged, as far as possible, the design thickness of the buffer material between the overpack and the rock surface of the emplacement hole.

While clays are excellent hydraulic and chemical barrier materials, many candidate clays, such as bentonite, have certain other properties that make it difficult to maintain the as-designed separation between the overpack and the wall of the emplacement hole. First, when contacted by groundwaters of appropriate chemical composition, $\mathrm{pH}$ and ionic strength, many clays increase in volume. This swelling occurs due to increased replusion between opposing surfaces of clay platelets resulting either from changes in the effective surface charge of the clay platelets, or from changes in the dielectric strength of the solution between them. If this tendency to swell is restrained by confining the clay between fixed surfaces, a compressive stress or "swelling pressure" is exerted on the confining surfaces. The swelling pressures that are generated can be quite large; large enough to overcome in-situ rock stresses and fracture the surface of an 


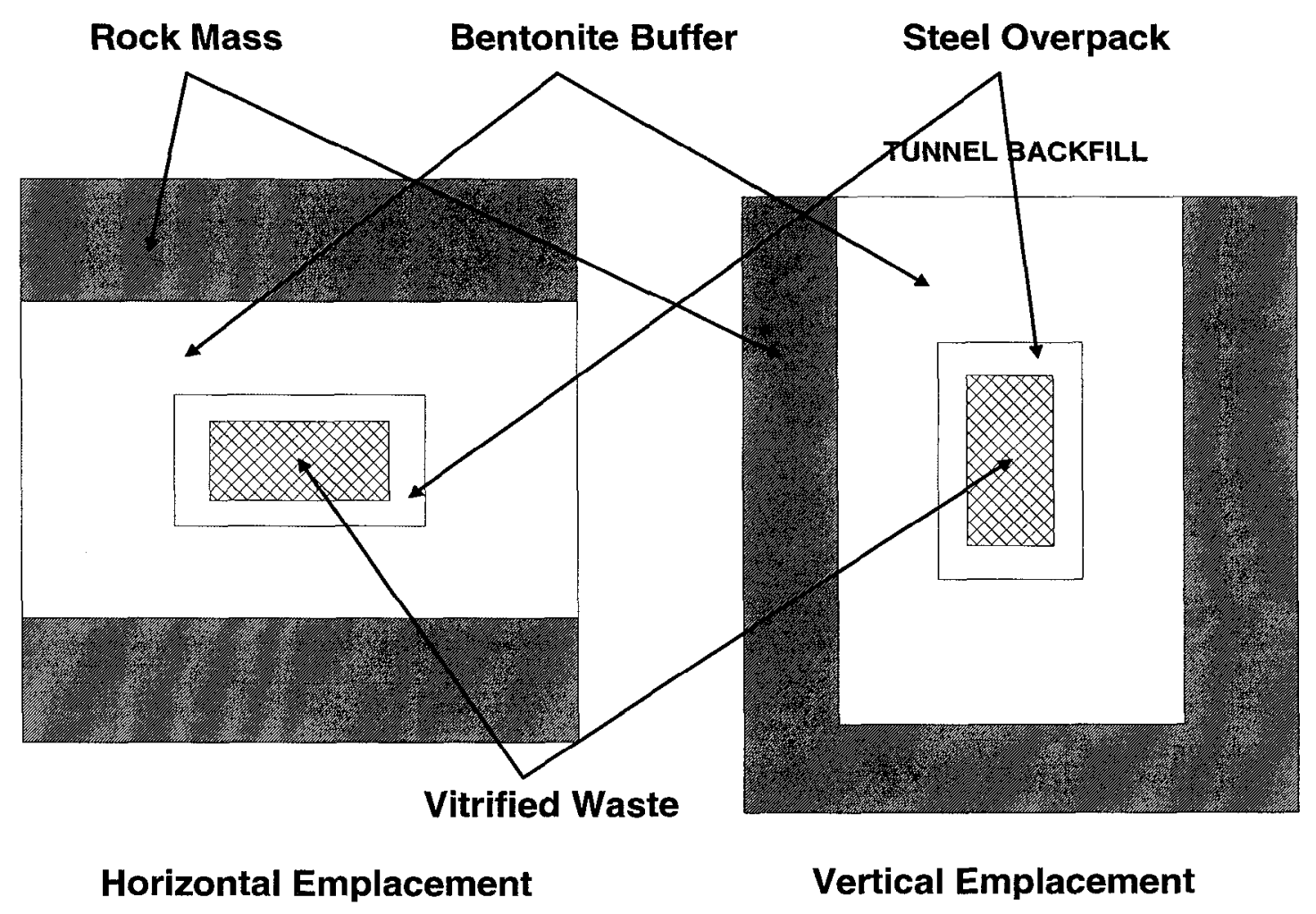

Figure 1.1-1: The Engineered Barrier System Concept 
emplacement hole, extrude clay into fractures, or cause deformation and/or movement of the overpack within an emplacement hole [2].

The second property of clay that causes difficulties in maintaining the design buffer thickness is the softening and loss of shear strength of clay upon contact with water. When contacted by water, clay loses shcar strength and undergoes plastic flow more readily, and may be more easily extruded into permeable fractures that intersect the walls of an emplacement hole. Under these conditions, an overpack emplaced in clay may settle and drift more easily towards the wall or floor of an emplacement hole either due to loss of shear strength, or due to extrusion of the underlying clay into fractures.

Another potential driving force for migration of an overpack emplaced in a clay buffer, is the increase in volume of the outer layers of the steel overpack due to corrosion by groundwater. Because the density of the corrosion products is less than one-third the density of steel, this increase in volume within the restricted space of an emplacement hole may cause sufficient pressure to induce plastic flow of the clay and migration of the overpack off-center in the hole. All of these mechanisms, that could cause the displacement of an overpack towards the wall of an emplacement hole and compromise the performance of the engineered barrier system, are depicted in Figure 1.1-2.

\subsection{OBJECTIVE \& APPROACH}

The ultimate objective of the work reported here is to develop the theoretical basis, and a robust computational method, to compute and evaluate the significance of the coupled processes of water transport and elasto-plastic deformation of clay in the design of an engineered barrier system for geologic disposal of radioactive waste. In particular, the model must be able to realistically represent the swelling, softening and frictional constitutive behaviors of clays and compute the extrusion of clay into thin fractures, and the migration of a steel overpack emplaced within an hole filled with clay buffer material.

The greatest difficulty in computing a problem of this kind, is that of accurately computing the large deformations that would occur at the entrance to a thin fracture into which clay is extruded under pressure. Standard, large-deformation finite element codes based on Lagrangian formulations suffer large distortions in the computational grid and require frequent manual intervention to stop the computation, regenerate a new grid, remap variables to the new grid, and restart the computation. However, the ALE-3D Arbitrary Lagrangian-Eulerian finite element computer code [3,4], selected for performing these computations, does that automatically at the end of every time step and invokes high-order algorithms to remap the physical varibles to the new mesh with high accuracy. It also has the capability to automatically extend its grid to accommodate newly generated contact surfaces, such as that between the extruded clay plug within a fracture and the fracture surfaces. 


\section{Horizontal Emplacement Cross-Section}

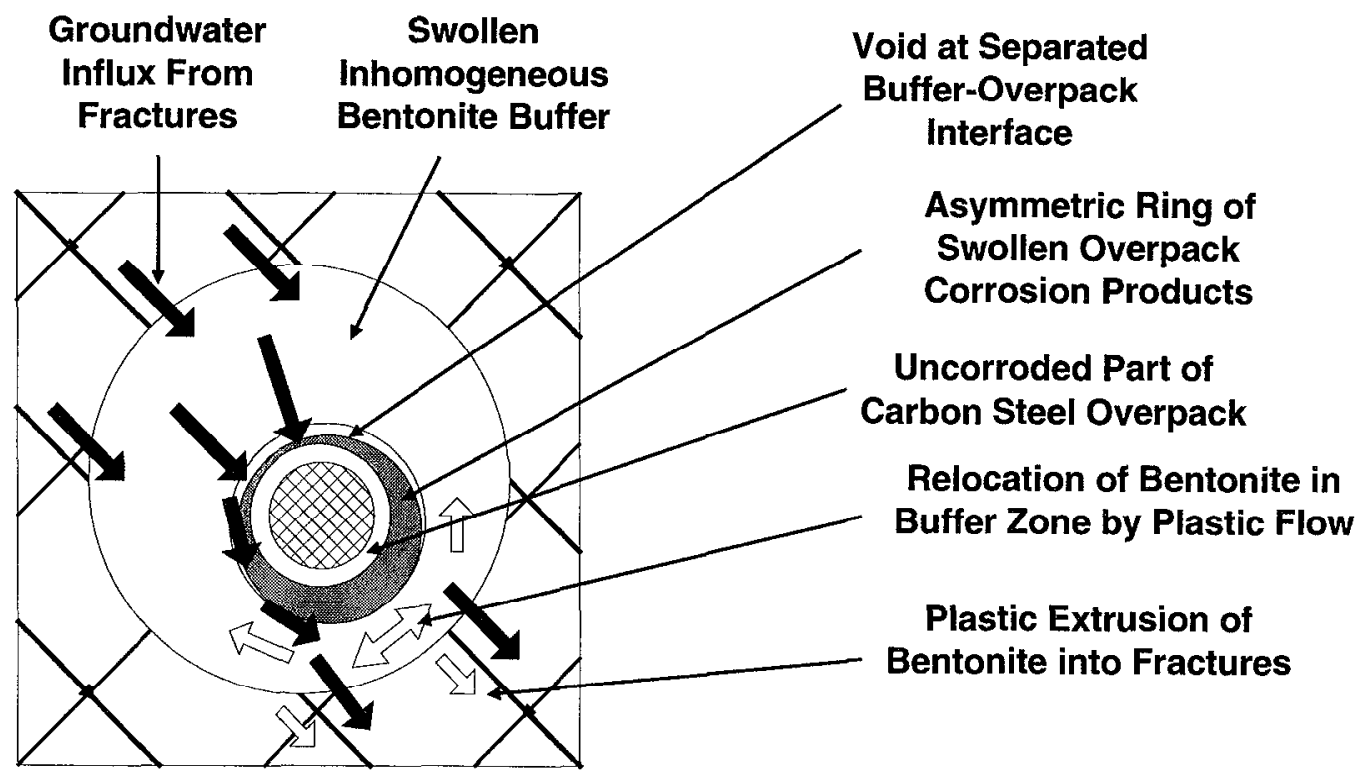

Figure 1.1-2: Mechanisms for Overpack Migration 
The existing elasto-plastic constitutive models in the ALE-3D code, which have been designed for metals deforming under dynamic transient conditions, need to be replaced with models that better represent the frictional behavior of clays subject to swelling and softening when contacted by water. Also, the transport of water within the clay buffer and the effects of stress development due to swelling can be modelled only approximately through the use of the existing coupled heat diffusion and thermo-elastoplasticity modeling capabilities of the ALE-3D code. Nevertheless, it is possible to approximately model most of the important processes with the existing ALE-3D code without any code enhancements.

The work in this Task will be carried out in four stages:

- Task 4.1: Demonstrate the mesh adaptation capability of the ALE-3D code (Year 1),

- Task 4.2: Demonstrate the capability of the ALE-3D code to compute realistic problems (Year 1),

- Task 4.3: Implement and verify improved elasto-plastic deformation models in the ALE-3D code (Year 2),

- Task 4.4: Estimate the timing and spatial distribution of rewetting and the resulting migration of an emplaced overpack (Year 2).

In the current funding year (Year 1), we developed a rigorous theoretical model for the coupled processes of elasto-plastic deformation and water transport in clay. Then we established the theoretical basis for the approximations needed to compute these processes using the ALE-3D code and computed three problems that would meet the goals of sub-Tasks 4.1 and 4.2. In view of the limited time and funds available for the work, and the unavailability of relevant input data, we selected generic problems with the simple physical configurations and problem specifications that would permit us to demonstrate the required capabilities of the ALE-3D code. Next year, we plan to enhance the ALE-3D code by embedding improved elasto-plastic constitutive models, and to extend these computations to more realistic problems using material property and physical geometry data that has become available recently from the Japanese repository program.

In Chapter 2 of this report, we present the theoretical model that was developed and identify the approximations that must be made to compute these problems using the ALE-3D code. This work also establishes the general approach to elasto-plastic constitutive modeling that will form the basis for the ALE-3D code enhancements to be implemented next year in sub-Task 4.3. In Chapter 3, the results of the three problems that were computed to demonstrate the capabilities of the ALE-3D code are presented. In Chapter 4, we outline the conclusions arrived at as a result of this work, and present our recommendations for the next phase of this Task. 


\section{MODEL FORMIULATION}

\subsection{THEORETICAL MODEL FORMULATION}

In this section we develop the theoretical basis and underlying approximations for using the ALE-3D code to simulate the coupled processes of clay deformation and unsaturated moisture transport in a clay-filled tunnel that contains an emplaced overpack bearing nuclear waste. The primary objective of these simulations is to demonstrate that the ALE-3D code is capable 1) of handling the large localized deformation of the clay at the entrances to rock fractures into which clay is extruded, and 2) of computing the coupled processes of moisture induced clay swelling, softening and deformation that result in displacement of the overpack within the tunnel. In doing so, we simplify the problem by neglecting the temperature changes due to the heat generated by the waste and the deformation of both the overpack and the surrounding rock mass.

The ALE-3D code is an Arbitrary Lagrangian-Eulerian code $[3,4]$ designed for computing problems that involve large deformation and heat flow in metallic components under highly-energetic dynamic conditions that exist in applications such as metal forming. Because of its Arbitrary Lagrangian-Eulerian numerical formulation, the ALE-3D code can easily cope with large deformations of solid materials that retain a memory of the undeformed state without the frequent prohibitively labor-intensive manual remapping of the grid required by purely Lagrangian computer codes. Although the material models currently available in the ALE-3D computer code do not include frictional geological materials such as clay and rock, by suitable choicc of parameters in an existing elasto-plastic model for metals, we were able to represent a Drucker-Prager type elasto-plastic material that exhibits frictional behavior. Internal material friction of this kind arises as a result of frictional contact and interlocking between the rough surfaces of the material particles, such as sand grains and clay domains, and is distinct from the external boundary friction that takes place between the material particles and external confining surfaces (i.e., external boundary friction). We utilized the heat diffusion and thermo-elastic strain modeling capabilities of the code to represent the effects of swelling due to water transport within the clay. We modified the ALE-3D computer code to include the softening of the clay upon being wetted by water. Furthermore, we were able to compute the largely quasi-static deformations in the problem of interest over large times, by appropriately scaling the inertia terms in the equations of motion to increase to practical levels the time-step size permitted by the explicit time-stepping method.

\subsubsection{Governing Field Equations}

We view the clay as a deforming porous solid whose pores are filled by liquid and gas phases which move relative to the deforming solid clay matrix. Each of these phases is assumed to form a distinct physical phase occupying its own volume with the solid, liquid and gas phases containing primarily clay, water and air, respectively. For simplicity, we assume that there is no mass transfer between these phases, and in 
particular, that the water vapor in the air phase does not have a significant impact on the wetting of clay. Under these assumptions we will hereafter refer to the liquid phase as the water phase and the gas phase as the air phase. The governing field equations are written for a deforming representative elementary volume (REV) enclosing a fixed mass of solid in terms of continuum average variables that are defined on a length scale much larger than the characteristic length of the pores. The field equations for the conservation of mass are written down separately for the solid, water and air phases. In contrast, the field equations for the conservation of linear momentum are written for the combined solid, water and air phases within the deforming REV, and for the separate water and air phases which move relative to the deforming solid. These equations are then supplemented by constitutive relationships for the elasto-plastic deformation of the clay, the swelling and softening of the clay when contacted by water, and the transport of the water and air under the action of gravitational, pressure, capillary and viscous forces. Finally, we invoke a number of assumptions to simplify the resulting set of field equations to a form that is supported by the ALE-3D code.

\section{Mass Balance Equations for Partially Saturated Deforming Porous Medium}

Assuming that there is no mass transfer between the solid, water and air phases, and neglecting diffusive and dispersive transport as small compared to the advective transport at the average phase flow velocities, the equations of conservation of mass for the solid, water and air phases can be written as

$$
\begin{aligned}
& \frac{1}{J} \frac{\partial\left[J(1-\phi) \rho_{s}\right]}{\partial t}=0 \\
& \frac{1}{J} \frac{\partial\left[J \phi S_{w} \rho_{w}\right]}{\partial t}+\frac{\partial\left[\rho_{w} \dot{W}_{w i}\right]}{\partial x_{i}}=0 \\
& \frac{1}{J} \frac{\partial\left[J \phi\left(1-S_{w}\right) \rho_{a}\right]}{\partial t}+\frac{\partial\left[\rho_{a} \dot{W}_{a i}\right]}{\partial x_{i}}=0
\end{aligned}
$$

where, $\mathrm{t}$ is the time variable, $x_{i}$ the spatial coordinate in $i$-th direction, and the subscripts $s, w$, and $a$ identify the water and air phases, respectively. In the above equations, and hereafter, repeated latin indices $i, j, k$ etc. imply summation over the range (Einstein's convention). Also, $\phi$ is the porosity of the solid matrix, and $\rho_{s}, \rho_{w}$, and $\rho_{a}$ are the densities of the solid, water and air phases, respectively, $S_{w}$ is the saturation of the water phase defined as the fraction of pore volume occupied by the water phase, and $J=$ $d V / d V_{0}$ is the ratio of the bulk volume in the deformed state ( $d V$ ) to that in the undeformed initial state of the solid matrix $\left(d V_{0}\right)$. Furthemore, we have defined the D'Arcy advective flow rates per unit bulk area components in the water and air phases within the pores relative to the solid matrix by

$$
\dot{W}_{w i} \equiv \phi S_{w}\left(\dot{u}_{w i}-\dot{u}_{s i}\right)
$$


The velocity differences on the right hand side of the above equations are the average

$$
\dot{W}_{a i} \equiv \phi\left(1-S_{w}\right)\left(\dot{u}_{a i}-\dot{u}_{s i}\right)
$$

advective flow velocities of each phase relative to the solid.

Integrating Equation (2.1-1) with respect to time between the initial undeformed state (denoted by by the subscript ' ${ }^{\circ}$ ') and the current time, we obtain the following alternative expression for the conservation of mass for the solid material

$$
\frac{d M}{d V_{0}}=(1-\phi) \rho_{s} J=\left(1-\phi_{0}\right) \rho_{s 0}
$$

where $d V_{0}$ is the initial bulk volume. Rearranging this equation, the porosity $\phi$ is given by

$$
\phi=1-\left(\frac{\left(1-\phi_{0}\right) \rho_{s 0}}{\rho_{s} J}\right)
$$

in terms of the solid density and the volume ratio $J$. At this time, it is useful to note the relationship

$$
\frac{1}{J} \frac{\partial J}{\partial t}=\frac{\partial \dot{u}_{s i}}{\partial x_{i}}
$$

that exists between the solid velocity and the rate of change of the volume ratio $J$. From Equations (2.1-1), (2.1-2), and (2.1-3) we derive the equation of conservation of mass for the bulk material as

$$
\frac{1}{J} \frac{\partial\left[J \rho_{b}\right]}{\partial t}+\frac{\partial\left[\rho_{w} \dot{W}_{w i}+\rho_{a} \dot{W}_{a i}\right]}{\partial x_{i}}=0
$$

where the bulk density $\rho_{b}$ of the solid, water and air mixture is defined by

$$
\rho_{b} \equiv\left[(1-\phi) \rho_{s}+\phi S_{w}, \rho_{w}+\phi\left(1-S_{w}\right) \rho_{a}\right] \quad(2.1-10)
$$

\section{Equations of Motion for Partially Saturated Deforming Porous Medium}

The equation for the conservation of momentum of the bulk material within the deforming REV containing a fixed mass of solid is given in differential form by

$$
\begin{aligned}
& \frac{1}{J} \frac{\partial}{\partial t}\left[J\left(\rho_{b} \dot{u}_{s i}+\rho_{w} \dot{W}_{w i}+\rho_{a} \dot{W}_{a i}\right]+\frac{\partial}{\partial x_{j}}\left[\rho_{w} \dot{u}_{w i} \dot{W}_{w j}+\rho_{a} \dot{u}_{a i} \dot{W}_{a j}\right]\right. \\
& =\frac{\partial \tau_{j i}}{\partial x_{i}}+\rho_{b} g_{i}
\end{aligned}
$$


where $\tau_{i j}$ is the total Cauchy stress tensor acting on the bulk surface area composed of all threc phases and $g_{i}$ is the component of the gravitational acceleration in the $i$-th direction.

The equations for the conservation of momentum for the water and air phases take the form

$$
\begin{aligned}
& \frac{1}{J} \frac{\partial}{\partial t}\left[J \phi S_{w} \rho_{w} \dot{u}_{w i}-J \rho_{w} \kappa_{s} \dot{W}_{w i}-J \rho_{w} \kappa_{w a}\left(\dot{W}_{w i}-\dot{W}_{a i}\right)\right]+\frac{\partial}{\partial x_{j}}\left[\rho_{w} \dot{u}_{w i} \dot{W}_{w j}\right] \\
& =\phi S_{w}\left[-\frac{\partial p_{w}}{\partial x_{i}}+\rho_{w} g_{i}-\frac{\mu_{w}}{k k_{r w}\left(S_{w}\right)} \dot{W}_{w i}\right] \\
& \frac{1}{J} \frac{\partial}{\partial t}\left[J \phi\left(1-S_{w}\right) \rho_{a} \dot{u}_{a i}-J \rho_{a} \kappa_{s} \dot{W}_{a i}-J \rho_{a} \kappa_{w a}\left(\dot{W}_{a i}-\dot{W}_{w i}\right)\right]+\frac{\partial}{\partial x_{j}}\left[\rho_{a} \dot{u}_{a i} \dot{W}_{a j}\right] \\
& =\phi\left(1-S_{w}\right)\left[-\frac{\partial p_{a}}{\partial x_{i}}+\rho_{a} g_{i}-\frac{\mu_{a}}{k k_{r a}\left(S_{w}\right)} \dot{W}_{a i}\right]
\end{aligned}
$$

where, $\kappa_{s}$ is the added-mass coefficient that represents the inertial coupling between a fluid phase and the solid phase, and $\kappa_{w a}$ is the added-mass coefficient that represents the inertial coupling between the water and gas phases, respectively. Furthermore, $p_{w}$ and $p_{a}$ are the water and air pressures, $\mu_{w}$ and $\mu_{a}$ are the shear viscosities of water and air, $k_{a}$ is the absolute permeability of the porous solid matrix, and $k_{r w}$ and $k_{r a}$ are the relative permeabilities to water and air of the porous solid matrix.

Although the inertia terms on the left-hand sides of these equations are not significant in the problems we intend to address here, we have retained these terms because the ALE-3D code uses these terms to numerically integrate the first equation in time by an explicit time-stepping method. Subsequently, we will make simplifying assumptions to eliminate most of these terms from the computational model.

\section{Capillary Pressure and Relative Permeability Relationships}

The water and air pressures are related through the capillary pressure relationship

$$
p_{a}-p_{w} \equiv p_{c a w}\left(S_{w}\right)
$$

where, the capillary pressure $p_{c a w}$ is a function of the the pore size distribution, the surface tension of the fluid interfaces, the wettability of the solid surfaces and the curvature of the fluid interfaces. While assuming that the pore size distribution (which may be altered by applied stresses) and the surface tension are represented through measured material parameters of the capillary pressure function, we have explicitly 
indicated dependence on the curvature of the fluid interfaces through the argument $S_{w}$ (i.e., water saturation). Likewise, the viscous drag forces between the flowing water and air and the solid clay matrix are strongly affected by the pore size distribution, surface tension, wettability and the curvature of the fluid interfaces. These drag forces, taken into account through the absolute permeability and fluid viscosity in single phase flow, have been modified to account for the effects of partial saturation by means of the relative permeability factors that depend strongly on the degree of water saturation $S_{w}$.

\section{Effective Stress Decomposition of the Total Stress Tensor}

The total stress tensor $\tau_{i j}$ in Equation (2.1-11) acts on all three constituent phases of the bulk material. To be able to model the elasto-plastic deformation of the solid clay phase by the developed stresses, we must identify that portion of the total stress that acts on the solid matrix. There are many ways in which this decomposition can be accomplished. The obvious volume fraction weighted decomposition of the fluid and solid stresses is not the best way to model the deformation of the matrix. This is because a particulate material is deformed by the forces exerted at the points of contact between the solid grains and grain-to-grain contact occurs over a smaller contact area than that given by the solid volume fraction. Under volume fraction weighting, the contribution of the total fluid pressure to the total stress is weighted by the porosity, whereas it should be assigned a greater weighting on the basis of the actual solid contact area that is smaller than the solid volume fraction. Because the solid contact area is generally an unknown parameter, we believe it is better to adopt the Terzhagi effective stress decomposition and assign a weighting of unity to the fluid presure. Accordingly, we define the effective stress $\sigma_{i j}$ deforming the solid matrix as the stress in excess of the total fluid pressure and adpot the total stress decomposition

$$
\tau_{i j} \equiv \sigma_{i j}+p_{f} \delta_{i j}
$$

where, $p_{f}$ is the total fluid pressure defined in terms of the water and air phase pressures by

$$
p_{f} \equiv S_{w} p_{w}+\left(1-S_{w}\right) p_{a}
$$

In Equation (2.1-15) we have adopted the convention that compressive normal stresses and strains are positive. For subsequent use, we eliminate the water pressure from Equation (2.1-16) using the capillary pressure relationship (2.1-14), and express the total fluid pressure as

$$
p_{f}=p_{a}-S_{w} p_{c a w}\left(S_{w}\right)
$$




\subsubsection{Hydro-Elasto-Plastic Model for Clay Deformation, Sweiling and Softening}

The deformation of granular materials, such as clay, is frictional in character. That is, they exhibit a yield strength in shear that increases with the compressive normal stress across the plane that is yielding in shear. The simplest yield criterion that exhibits this type of behavior is the Mohr-Coulomb frictional yield criterion in which the minimum shear strength at zero compressive strength is equal to the cohesion $c$ and the yield strength linearly increases above this by an additional amount equal to the cnefficient of friction $\mu=\tan \varphi_{\mu}$ multiplied by the normal compressive stress. Here, $\varphi_{\mu}$ is the internal friction angle of the granular material.

$$
\sigma_{s}=c+\mu \sigma_{n}
$$

The Standard Drucker-Prager elasto-plastic model [5] 1) extends the MohrCoulomb model to multidimensional stress states, and 2) accounts for continued plastic flow with plastic hardening when loaded beyond the initial yield stress under multidimensional stress states. In the Extended Drucker-Prager elasto-plastic model that we present here, we introduce novel extensions to the Standard Drucker-Prager elastoplastic model to include the effects of 3) swelling expansion of the porous solid when contacted by water, and 4) the softening of the clay and reduction in the elastic stiffness and plastic-yield strength when the clay is contacted by water.

We begin by defining the deviatoric effective stress $S_{i j}$ by

$$
S_{i j} \equiv \sigma_{i j}-\frac{1}{3} \sigma_{k k} \delta_{i j}
$$

where, as before, repeated Latin indices indicate summation over the range (1-3) and $\delta_{i j}$ $(-0, i \neq j ;-1 i-j)$ is the Kronecker delta function. The Extended Drucker-Prager yield criterion $F$, with fluid saturation dependent cohesion $c_{F}\left(S_{w}\right)$ and frictional $\mu_{F}\left(S_{w}\right)$ parameters is given by

$$
F\left(\sigma_{i j}, S_{w}\right) \equiv \sqrt{J_{s 2}}-\mu_{F}\left(S_{w}\right) J_{\sigma 1}-c_{F}\left(S_{w}\right)=0
$$

where, the first invariant $J_{\sigma I}$ of the effective stress $\sigma_{i j}$, and the second invariant $J_{s 2}$ of the deviatoric effective stress $S_{i j}$, are defined by

$$
J_{\sigma 1} \equiv \sigma_{k k} \quad ; \quad J_{s 2} \equiv \frac{1}{2} S_{k l} S_{k l}
$$

The first invariant $J_{\sigma l}$ of the effective stress is equal to the three times the average normal stress; it is, therefore, the multidimensional analog of the normal stress in the MohrCoulomb yield criterion. The second invariant $J_{s 2}$ of the deviatoric effective stress, is free 
of normal stress; its square root in Equation (2.1-20) is the mutidimensional analog of the effective shear stress in the Mohr-Coulomb yield criterion. The generalized cohesion $c_{F}\left(S_{w}\right)$ and frictional $\mu_{F}\left(S_{w}\right)$ parameters in this Equation must be properly interpreted and related to the cohesion and friction angle parameters measured under test conditions. For example, if the cohesion $c$ and friction angle $\phi_{\mu}$ are measured in a conventional triaxial compression test, the generalized cohesion $c_{F}$ and friction coefficient $\mu_{F}$ parameters are given by

$$
c_{F}=\frac{6 c \operatorname{Cos} \phi_{\mu}}{\sqrt{3}\left(3-\operatorname{Sin} \phi_{\mu}\right)} ; \mu_{F}=\frac{2 \operatorname{Sin} \phi_{\mu}}{\sqrt{3}\left(3-\operatorname{Sin} \phi_{\mu}\right)}(2.1-22 a, b)
$$

\section{Strain Decomposition}

The total strain increment $d \varepsilon_{i j}$ induced by an incremental increase in effective stress $d \sigma_{i j}$ during elasto plastic deformation is the sum of an elastic strain increment $d \varepsilon_{i j}^{e}$, a plastic strain increment $d \varepsilon_{i j}^{p}$, and a swelling induced strain increment $d \varepsilon_{i j}^{s}$

$$
d \varepsilon_{i j} \equiv \frac{1}{2}\left(\frac{\partial u_{s i}}{\partial x_{j}}+\frac{\partial u_{s j}}{\partial x_{i}}\right)=d \varepsilon_{i j}^{e}+d \varepsilon_{i j}^{p}+d \varepsilon_{i j}^{s}
$$

The elastic strain increment is assumed to be related to the stress increment through fluid saturation dependent linear isotropic shear and bulk compressive elastic moduli $G$ and $K$, while the swelling strain increment is assumed to be isotropic and to be equal to the incremental increase in fluid saturation multiplied by a swelling expansion coefficient $\beta$. Thus,

$$
\begin{aligned}
& d \sigma_{i j}=2 G d \varepsilon_{i j}^{e}+\left(K-\frac{2}{3} G\right) d \varepsilon_{k k}^{e} \delta_{i j} \\
& d \varepsilon_{i j}^{s}=-\frac{1}{3} \beta d S_{w} \delta_{i j} \quad(2.1-25)
\end{aligned}
$$

where, consistent with assumptions regarding normal effective stress, the compressive normal strains are assumed to be positive

\section{Plastic Strain Increment}

To determine the plastic strains induced by the incremental changes in stress and in water saturation, we first utilize the condition that while the material is yielding, the stress state must remain on the yield surface in stress space. Equation (2.1-20) must, therefore, be satisfied and the incremental change in the yield function must vanish. Thus,

$$
d F=\frac{\partial F}{\partial \sigma_{i j}} d \sigma_{i j}+\frac{\partial F}{\partial S_{w}} d S_{w}=0
$$


Generaily, the material while yielding is assumed to suffer plastic flows to an extent that is determined by a flow potential function. If the flow potential function is assumed to be the same as the yield function, the plastic flow is said to be associated. For simplicity, we

$$
d F=\left(\frac{S_{i j}}{2 \sqrt{J_{s 2}}}-\mu_{F} \delta_{i j}\right) d \sigma_{i j}-\left(\frac{\partial \mu_{F}}{\partial S_{w}} J_{\sigma l}+\frac{\partial c_{F}}{\partial S_{w}}\right) d S_{w}=0 \quad(2.1-27)
$$

assume here that the plastic flow is Associated and derive the plastic strain increments from this function according to

$$
d \varepsilon_{i j}^{p}=\frac{\partial F}{\partial \sigma_{i j}} d \lambda=\left(\frac{S_{i j}}{2 \sqrt{J_{s 2}}}-\mu_{F} \delta_{i j}\right) d \lambda(2.1-28)
$$

where, $d \lambda$ is an as yet undetermined magnitude of the multidimensional plastic flow vector. Substituting for the swelling and plastic strain increments in Equation (2.1-23), and solving for the elastic strain increment we obtain

$$
d \varepsilon_{i j}^{e}=d \varepsilon_{i j}+\frac{1}{3} \beta d S_{w} \delta_{i j-}\left(\frac{S_{i j}}{2 \sqrt{J_{s 2}}}-\mu_{F} \delta_{i j}\right) d \lambda(2.1-29 a)
$$

Contracting the above Equation for $i=j=k$ and noting that $S_{k k}=0$, we have

$$
d \varepsilon_{k k}^{e}=d \varepsilon_{k k}+\beta d S_{w}-3 \mu_{F} d \lambda(2.1-29 b)
$$

Substituting from the above two Equations for the elastic strains in Equation (2.1-24), we obtain the following relationship for the effective stress increments

$$
d \sigma_{i j}=2 G d \varepsilon_{i j}+\left(K-\frac{2}{3} G\right) d \varepsilon_{k k} \delta_{i j}+\left(3 K \mu_{F} \delta_{i j}-G \frac{S_{i j}}{\sqrt{J_{s 2}}}\right) d \lambda+K \beta d S_{w} \delta_{i j}
$$

Now, we substitute for $d \sigma_{i j}$ in Equation (2.1-27) using the above Equation and solve for the magnitude $d \lambda$ of the plastic flow vector magnitude. After considerable algebraic manipulation, and utilizing the properties of the deviatoric stress tensor $S_{i j}$ to simplify the expressions, we obtain the result

$$
d \hat{\lambda}=\Lambda_{\varepsilon k l} d \varepsilon_{k l}-\Lambda_{s} d S_{w}
$$

where, plastic strain vector coefficients are defined by the expressions 


$$
\begin{gathered}
\Lambda_{\varepsilon k l} \equiv \frac{\sigma_{k l}-\left(\frac{J_{\sigma 1}}{3}+\frac{3 K \mu_{F} \sqrt{J_{s 2}}}{G}\right) \delta_{m k} \delta_{m l}}{\sqrt{J_{s 2}\left(1+\frac{9 \mu_{F}^{2} K}{G}\right)}} \\
\Lambda_{s} \equiv \frac{\left(3 K \mu_{F^{\prime}} \beta+\frac{\left.\partial \mu_{F^{\prime}} J_{\sigma 1}+\frac{\partial c_{F^{\prime}}}{\partial S_{w}}\right)}{G\left(1+\frac{9 \mu_{F}^{2} K}{G}\right)}\right.}{G\left(1+\frac{S_{w}}{G}\right)}
\end{gathered}
$$

\section{Constitutive Relationship for Elasto-Plastic Deformation, Swelling and Softening}

The total effective stress increment $d \sigma_{i j}$ can now be expressed in terms of the total strain increment $d \varepsilon_{i j}$ and the water saturation increment $d S_{w}$ by using Equation (2.1-31) to eliminate the plastic strain vector magnitude $d \lambda$ from Equation (2.1-30)

$$
\begin{aligned}
d \sigma_{i j} & =2 G d \varepsilon_{i j}+\left(K-\frac{2}{3} G\right) d \varepsilon_{k k} \delta_{i j}+\left(3 K \mu_{F} \delta_{i j}-G \frac{S_{i j}}{\sqrt{J_{s 2}}}\right) \Lambda_{\varepsilon k l} d \varepsilon_{k l} \\
& +\left(K \beta \delta_{i j}-\left(3 K \mu_{F} \delta_{i j}-G \frac{S_{i j}}{\sqrt{J_{s 2}}}\right) \Lambda_{s}\right) d S_{w}
\end{aligned}
$$

This constitutive relationship for elasto-plastic deformation, swelling and softening of clay can be recast in the more compact form

$$
d \sigma_{i j}=E_{i j k l}^{\varepsilon} d \varepsilon_{k l}+E_{i j}^{s} d S_{w}
$$

where, the elasto-plastic deformation modulus $E_{i j k l}^{i}$ and the elasto-plastic swellingsoftening modulus $E_{i j}^{s}$ are given by the definitions

$$
\begin{gathered}
E_{i j k l}^{\mathcal{E}} \equiv 2 G \delta_{i k} \delta_{j l}+\left(K-\frac{2}{3} G\right) \delta_{i j} \delta_{m k} \delta_{m l}+\left(3 K \mu_{F} \delta_{i j}-G \frac{S_{i j}}{\sqrt{J_{s 2}}}\right) \Lambda_{\varepsilon k l} \\
E_{i j}^{S} \equiv K \beta \delta_{i j}-\left(3 K \mu_{F} \delta_{i j}-G \frac{S_{i j}}{\sqrt{J_{s 2}}}\right) \Lambda_{s}
\end{gathered}
$$


We immediately recognize the first two terms of Equations (2.1-34) and (2.1-35a) as the purely elastic contributions to deformation. The first term in Equation (2.1-35b) is the hydroelastic contribution from swelling expansion that is analogous to the contribution from thermal expansion in the theory of thermoelasticity. The third term in Equation (2.1-35a) represents the plastic contribution from frictional Drucker-Prager type plasticity that, except for the dependence of the shear and bulk elastic moduli $G\left(S_{w}\right)$ and $K\left(S_{w}\right)$ on water saturation, is independent of water induced swelling and softening. The second term in Equation (2.1-35b) represents the coupled effects of frictional plastic deformation, water induced swelling of the clay, and the water induced softening of the frictional and cohesion parameters that determine the yield strength of the clay according to the present Extended Drucker-Prager plasticity model.

\subsubsection{Approximate Model for ALE-3D Code Computations}

\section{Solid Deformation}

The general mathematical model developed so far must be simplified further to permit simulation by the ALE-3D code. The final objective of these approximations is to derive a diffusion equation for the movement of water within the clay and an elastoplastic equation of motion for the solid matrix displacements that is coupled to the water saturation through a swelling expansion term. We begin by using Equations (2.1-9) and (2.1-15) to re-write Equation (2.1-11) in the form

$$
\begin{aligned}
& \rho_{b} \frac{\partial \dot{u}_{s i}}{\partial t}+\frac{1}{J} \frac{\partial}{\partial t}\left[\rho_{w} \dot{W}_{w i}+\rho_{a} \dot{W}_{a i}\right]+\frac{\partial}{\partial x_{j}}\left[\rho_{w} \dot{u}_{w i} \dot{W}_{w j}+\rho_{a} \dot{u}_{a i} \dot{W}_{a j}\right] \\
& +\frac{\partial \dot{u}_{s j}}{\partial x_{j}}\left[\rho_{w} \dot{W}_{w i}+\rho_{a} \dot{W}_{a i}\right]-\dot{u}_{s i} \frac{\partial}{\partial x_{j}}\left[\rho_{w} \dot{W}_{w j}+\rho_{a} \dot{W}_{a j}\right] \\
& =\frac{\partial \sigma_{j i}}{\partial x_{i}}+\frac{\partial p_{f}}{\partial x_{i}}+\rho_{b} g_{i}
\end{aligned}
$$

We now make the assumptions that, compared to the first term, all other inertia terms on the lefthand side of the equation of motion for the bulk porous material (Equation (2.136)) are small, and that all inertia terms on the lefthand sides of equations of motion for the water and air phases (Equations (2.1-12), (2.1-13)) can be neglected. Thus, with some re-arranging of terms, we obtain

$$
\rho_{b} \frac{\partial \dot{u}_{s i}}{\partial t} \approx \frac{\partial \sigma_{j i}}{\partial x_{i}}+\frac{\partial p_{f}}{\partial x_{i}}+\rho_{b} g_{i}
$$




$$
\begin{aligned}
& \dot{W}_{w i} \approx \frac{k k_{r w}\left(S_{w}\right)}{\mu_{w}}\left[-\frac{\partial p_{w}}{\partial x_{i}}+\rho_{w} g_{i}\right] \\
& \dot{W}_{a i} \approx \frac{k k_{r a}\left(S_{w}\right)}{\mu_{a}}\left[-\frac{\partial p_{a}}{\partial x_{i}}+\rho_{a} g_{i}\right]
\end{aligned}
$$

It should be noted that the first inertia term in all three equations are of the same order of magnitude and are negligible compared to the individual terms on the righthand side. We have retained the first inertia term in Equation (2.1-37) only because it is needed for integration of the resulting equations in the ALE-3D code. This will not affect the accuracy of the computations, becausc inertia effects are always negligible in the problems we address here.

We now make the important assumption that because the movement of water and air within the clay matrix is very slow, and the viscosity of air is also very small, the pressure of the air phase is uniform throughout the clay and is constant in time. Because the pressure of air is contanstant by assumption, we will require to model the movement of air using Equations (2.1-3) and (2.1-39). As a result of this assumption, the total pressure given by Equation (2.1-17) is solely a function of the water saturation in the clay. We now substitute in Equation (2.1-37) for the effective stress increment using Equation (2.1-34) and for the total pressure using Equation (2.1-17), and obtain

$$
\rho_{b} \frac{\partial \dot{u}_{s i}}{\partial t} \approx E_{j i k l}^{\varepsilon} \frac{\partial \varepsilon_{k l}}{\partial x_{j}}-\left(E_{j i}^{s}+\frac{\partial}{\partial S_{w}}\left(S_{w} p_{c a w}\right) \delta_{j i}\right) \frac{\partial S_{w}}{\partial x_{j}}+\rho_{b} g_{i}(2.1-40)
$$

To facilitate the use of the thermo-elasto-plastic model in the ALE-3D computations for our purposes we identify fluid saturation as the analog of temperature, and diffusive fluid transport as the analog of diffusive heat conduction. Towards this end, Equation (2.1-40) is further simplified by neglecting the capillary pressure term and approximating the second term in the definition of $E_{i j}^{s}$ related to the parameter $\Lambda_{s}$. Thus, the thermo-elastoplastic model used in ALE-3D computations, and represented by Equation (2.1-41)

$$
\rho_{b} \frac{\partial \dot{u}_{S i}}{\partial t} \approx E_{j i k l}^{\varepsilon} \frac{\partial \varepsilon_{k l}}{\partial x_{j}}-K \beta \delta_{j i} \frac{\partial S_{w}}{\partial x_{j}}+\rho_{b} g_{i}(2.1-41)
$$


below, does not include the full impact of water-induced swelling and softening represented by the Extended Drucker-Prager model.

\section{Water Transport}

The equation for water transport obtained by combining the water mass conservation Equation (2.1-2), and Equation (2.1-38) for the D'Arcy water flow velocity under unsaturated two-phase flow conditions, is given by

$$
\frac{1}{J} \frac{\partial}{\partial t}\left(J \phi S_{w} \rho_{w}\right) \approx \frac{\partial}{\partial x_{k}}\left[\frac{k k_{r w}\left(S_{w}\right) \rho_{w}}{\mu_{w}}\left(-\frac{\partial p_{w}}{\partial x_{k}}+\rho_{w} g_{k}\right)\right]
$$

Using Equation (2.1-14) and the assumption of constant air pressure to eliminate water pressure in favor of water saturation in the above Equation, we obtain

$$
\frac{1}{J} \frac{\partial}{\partial t}\left(J \phi S_{w} \rho_{w}\right) \approx \frac{\partial}{\partial x_{k}}\left[\phi D_{w} \rho_{w} \frac{\partial S_{w}}{\partial x_{k}}+\frac{k k_{r w}\left(S_{w}\right) \rho_{w}^{2}}{\mu_{w}} \rho_{w} g_{k}\right] \text { (2.1-43) }
$$

where we have defined capillarity driven water diffusivity $D_{w}$ in the clay by

$$
\phi D_{w}\left(S_{w}\right) \equiv-\frac{k k_{r w}}{\mu_{w}} \frac{\partial p_{c a w}}{\partial S_{w}}
$$

It can be shown through orders-of-magnitude estimation that

$$
\left(\begin{array}{cc}
S_{w} & \partial \\
J & \partial t
\end{array}\left(J \phi \rho_{w}\right)\right) /\left(\phi \rho_{w} \begin{array}{cc}
\partial S_{w} \\
& \partial t
\end{array}\right) \sim \beta \ll 1
$$

and that the effect of water compressibility is negligible (i.e., $\rho_{w} \approx$ constant) when compared to the effect of changes in water saturation. Consequently, Equation (2.1-43) can be reduced to a diffusion equation for water saturation given by

$$
\frac{\partial S_{w}}{\partial t} \approx \frac{\partial}{\partial x_{k}}\left[D_{w} \frac{\partial S_{w}}{\partial x_{k}}\right]
$$

The final model used in the ALE-3D computations is based on Equation (2.1-41) for coupled elasto-plastic deformation, swelling and softening of clay due to contact with water, on Equation (2.1-46) for the capillarity-driven diffusive transport of water within the clay, and on Equation (2.1-20) for the determination of the elasto-plastic state of the clay. 


\subsection{ALE-3D CODE SELECTION AND APPLICATION}

\subsubsection{Selection of the ALE-3D Code}

Two codes were considered for performing the computations presented in this report. These were the NIKE 2D/3D codes and the ALE-3D code [3]. The NIKE codes are Lagrangian codes in which the computational grid deforms with the deforming material. To avoid errors, whenever the grid deformation becomes excessive, the computation is stopped, the deformed domain is remeshed, the computational variables are manually remapped onto the new undistorted grid, and the calculation is resumed. When the remapping is frequent, it is very labor intensive and error prone. In contrast, in the ALE-3D Arbitrary Lagrangian-Eulerian code this remapping process is handled through high-order conservative algorithms that are automatically executed every time step with the result that the grid remains essentially the same as the initial grid. In addition, the capability exists for automatically refining or coarsening the grid locally to maintain the required levels of accuracy and computational efficiency. Furthermore, in the NIKE codes to couple a diffusive transport process (e.g., capillarity-dominated porous media water transport or heat conduction) to deformation, two separate codes for deformation and water flow/heat flow must be run, whereas both processes are simultaneously computed within one code by ALE-3D.

However, the NIKE codes include a wider array of elasto-plastic models for frictional geological materials, including Drucker-Prager models [5] and SandlerDimaggio "Cap" models [6]. The elasto-plastic models in the ALE-3D code are primarily designed for metal behavior under dynamically loaded conditions for use in metal forming applications [4], and are of limited applicability to geological materials. However, although designed for representing entirely different effects in energetic metal forming, several models for metals in the ALE-3D code can be made to represent a Drucker-Prager type elasto-plastic model through judicious selection of model parameters. Model extensions to model geological materials more accurately are possible, but were beyond the scope of the current work. Both the NIKE and ALE-3D codes have the capability to represent bonded, frictionless, and frictional interfaces through the use of slide surfaces. This capability is needed to model slipping of clay at tunnel, overpack and fracture surfaces.

One of the main issues of interest to the JNC in this Task was the capability to compute the extrusion of clay into thin fractures. This process exhibits large local deformations in the neighborhood of the entrances to the fractures that can be handled more efficiently by the ALE-3D code with its embedded Arbitrary Lagrangian-Eulerian automatic remeshing capability. Furthermore, the ease of modeling the diffusive transport of water within the same code, and incorporating its effect on the stress state through the existing capability for modeling thermoelastic expansion, made the ALE-3D code the better choice. Therefore, given the limited time and funds available to perform the required calculations, it was decided to perform the calculations with the ALE-3D code. 


\subsubsection{Application of the ALE-3D code}

In this Section we first describe how the Steinberg-Guinan elasto-plastic model for metals [7] was configured to represent a Drucker-Prager elasto-plastic model applicable to clay. Next we present how the inertia term in the equation of motion for the solid was scaled to allow large explicit time steps to be used without violating stability criteria that constrain the explicit time stepping procedure when computing the essentially quasi-static problems over large time periods.

\section{Reduction of the Steinberg-Guinan Model to Drucker-Prager Model}

The yield function of the Steinberg-Guinan elasto-plastic deformation model [7] available in the ALE-3D code is given by

$$
Y=Y_{0} f\left(\varepsilon_{p}\right) \frac{G(P, T)}{G_{0}}
$$

where,

$$
\begin{gathered}
Y_{0} f\left(\varepsilon_{p}\right) \equiv Y_{0}\left(1+\beta_{f}\left(\varepsilon_{p}+\varepsilon_{i}\right)\right)^{n} \leq Y_{\max } \\
G(P, T) \equiv G_{0}\left(1+A P \eta^{-1 / 3}-B(T-300)\right)
\end{gathered}
$$

In Equation (2.2-1), $Y$ is the yield shear stress, $\varepsilon_{p}$ is the plastic strain, $P$ is the average normal stress, $T$ is the temperature, $G(P, T)$ is the elastic shear modulus, $G_{0}$ is the initial shear modulus and the remaining quantities are material parameters of the elasto-plastic model. By identifying and selecting these variables and material parameters such that

$$
\sqrt{J_{s 2}}=Y ; \quad J_{s 1}=3 P ; \quad c_{F}=Y_{0} ; \quad \mu_{F}=Y_{0} A / 3 ; \quad \beta_{f}=0 ; \quad \eta=1 ; \quad B=0 \quad(2.2-4)
$$

we recover the Drucker-Prager yield criterion given by Equation (2.1-20). By allowing $Y_{o}$ and $A$ to decrease with water saturation, we are able to simulate softening of the clay due to wetting by water. In addition, however, we obtain a shear modulus that increases with the average normal stress and varies with water saturation according to the ratio $\left(\mu_{F} / c_{F}\right)$. This is a situation that is quite realistic for a granular material such as clay, and consistent with the Extended Drucker-Prager model that was derived previously.

$$
G\left(J_{s 1}, S_{w}\right) \equiv G_{0}\left(1+\frac{\mu_{F}\left(S_{w}\right)}{c_{F}\left(S_{w}\right)} J_{s 1}\right)
$$




\section{Inertia/Mass Scaling for Larger Explicit Time Steps}

As mentioned previously, the problems considered here involve essentially quasistatic processes that occur over time periods much larger than the characteristic time for propagation of inertial effects over the physical domain. However, we propose to utilize the inetia term to integrate Equation (2.1 -41) with an explicit time stepping procedure, by scaling the bulk density in the inertia term by a large factor $f_{\rho}$ that must meet certain criteria to maintain accuracy of the solution to the original problem of interest. In the absence of this factor, the time step size is limited by the Courant stability criterion which asserts that temporal changes during the computation must not outrun the wave speed. That is, if the shear wave speed $c_{s} \equiv\left(G / \rho_{b}\right)^{1 / 2}$ is an an estimate of the relevant wave speed, then we have the very restrictive condition

$$
\Delta t<\frac{\Delta x}{c_{s}} \quad(2.2-6)
$$

If on the other hand, we replace Equation (2.1-41) with

$$
f_{\rho} \rho_{b} \frac{\partial \dot{u}_{s i}}{\partial t} \approx E_{j i k l}^{\varepsilon} \frac{\partial \varepsilon_{k l}}{\partial x_{j}}-K \beta \delta_{j i} \frac{\partial S_{w}}{\partial x_{j}}+\rho_{b} g_{i} \quad(2.2-7)
$$

then, the stability criterion

$$
\Delta t \ll \sqrt{f_{\rho}} \frac{\Delta x}{c_{s}}
$$

can be made to be much less restrictive on time step size by making the density scaling factor $f_{\rho}$ sufficiently large.

However, for the solution to the original physical problem to remain essentially unaltered, the value of the density scaling factor cannot be arbitrarily large and must be sufficiently small for the inertia term in Equation (2.1-7) to remain much smaller than the terms on the lefthand side. The driving force in Equation (2.2-7) is the swelling expansion term which is proportional to the gradient of the water saturation established through diffusive water transport governed by Equation (2.1-46). To obtain an estimate of this gradient, we assume that the gradients are established over a distance $\delta$ in a time $\Delta t$. From Equation (2.1-46), we estimate

$$
\delta \sim \sqrt{D_{w} \Delta t} \quad(2.2-9)
$$


Using this distance $\delta$ to estimate the spatial gradients on the righthand side of Equation (2.2-7), and by balancing the first term on the righthand side against the swelling expansion gradient term, we obtain the following estimate for the changes in displacements $\Delta u$

$$
\Delta u \sim \beta \delta \Delta S_{w} \quad(2.2-10)
$$

We now demand that the scaled inertia term in Equation (2.2-7) be much smaller than the terms on the righthand side. That is, we require that

$$
\frac{\left(f_{\rho} \rho_{b} \frac{\partial \dot{u}_{s i}}{\partial t}\right)}{\left(K \beta \delta_{j i} \frac{\partial S_{w}}{\partial x_{j}}\right)} \ll<
$$

Using Estimates (2.2-9), (2.2-10) and the definition of wave speed, we have

$$
\frac{\left(f_{\rho} \rho_{b} \frac{\Delta u}{\Delta t^{2}}\right)}{\left(G \beta \frac{\Delta S_{w}}{\delta}\right)} \sim f_{\rho}\left(\frac{\delta}{c_{s} \Delta t}\right)^{2} \sim f_{\rho}\left(\frac{D_{w}}{c_{S}^{2} \Delta t}\right) \ll 1
$$

Re-arranging the above inequality, for the inertia term to be negligible, we must have

$$
\Delta t \gg f_{\rho} \frac{D_{w}}{c_{s}^{2}} \quad(2.2-12)
$$

Combining this requirement on the time step size, with the Courant stability requirement given by $(2.2-8)$ we obtain the result

$$
\Delta t_{\max } \equiv \sqrt{f_{\rho}} \frac{\Delta x}{c_{s}}>\Delta t>\Delta t_{\min } \equiv f_{\rho} \frac{D_{w}}{c_{s}^{2}}
$$

that establishes upper and lower bounds on the time step size for stable and physically accurate explicit time integration. Furthermore, (2.2-13) requires that the density scaling factor $f_{\rho}$. satisfy the following inequality for the inertia terms to remain negligible. 


$$
f_{\rho} \ll f_{\rho \max } \equiv\left(\frac{c_{s} \Delta x}{D_{w}}\right)^{2}
$$

Finally, it is useful to express the ratio between the lower and upper time step limits as

$$
\frac{\Delta t_{\max }}{\Delta t_{\min }}=\sqrt{\frac{f_{\rho \max }}{f_{\rho}}}
$$

Estimates show that the upper bound of the density scaling factor is very large for typical clay material properties and grid size dimensions. For example, for the values $G=$ $214 \mathrm{MPa}, \rho_{b}=1,800 \mathrm{Kg} / \mathrm{m}^{3}, D_{w}=1.0 \times 10^{-9} \mathrm{~m}^{2} / \mathrm{sec}$ and $\Delta x=0.01 \mathrm{~m}$, we obtain $\mathrm{c}_{\mathrm{s}}=345$ $\mathrm{m} / \mathrm{sec}$ and $f_{\text {pmax }}=12 \times 10^{18}$. Equation $(2.2-15)$ can then be used to estimate a suitable density scaling factor that would maintain an adequate margin of safety between the minimum and maximum allowed time step sizes. For example, if we wish to have maximum and minimum time step sizes that differ by a factor of 100, then from Equation $(2.2-15)$ the density scaling factor that should be used to achieve this result is $12 \times 10^{14}$. From Equation (2.2-13) we calculate the minimum and maximum time step sizes for this value of the density scaling factor to be $10 \mathrm{sec}$ and $1,000 \mathrm{sec}$, respectively. These values should be compared with the maximum time step size limit of $0.29 \times 10^{-4} \mathrm{sec}$ imposed by the normal Courant stability criterion in the absence of density scaling.

The computational efficiency of explicit time integration compared to implicit approaches depends strongly on the degree of non-linearity of the problem being solved. Alhough the allowed time step sizes with even density scaling are small compared to typical simulation periods of hundreds of years, in moderately non-linear problems, the total computational effort and time expended is often less than in implicit timeintegration because explicit calculations avoid the generation of iteration matrices and require far fewer calculations per time step. 


\subsection{COMPUTATIONAL RESULTS}

\subsection{Problem 1: EXTRusion of Clay INTO A FRACTURE dUE TO AN EXTERNALLY APPLIED STRESS}

The objective of Problem 1 is to meet the requirements of sub-Task 4.1 of this project by demonstrating the capability of the ALE-3D code to model clay extrusion into fractures under conditions when clay-extrusion is driven by un-coupled mechanical loading of the clay backfill. The primary purpose of this computation is to demonstrate the ability of the ALE-3D code to handle the extreme distortion of the clay at the entry to the fracture because of its automatic grid re-meshing capability.

\subsubsection{Assumptions \& Input Data}

This Problem is modeled in two spatial dimensions as shown in Figure 3.1-1 within a $0.5-\mathrm{m}$ wide $\mathrm{x} 0.5-\mathrm{m}$ tall, rigid-walled tunnel of rectangular cross-section. The tunnel is completely filled with a clay material at a uniform initial water saturation (0.47), airphase pressure $(1 \mathrm{~atm})$ and temperature $(25 \mathrm{deg}$. C). A single $1-\mathrm{mm}$ wide rigid-walled vertical fracture is assumed to intersect the tunnel floor at its center plane. Initially, the fracture is free of clay and is occupied by air at the initial pressure of the air-phase within the clay. The initial stress state within the clay is taken to be uniform and isotropic.

Table 3.1-1: Problem 1 - Input Model Parameter Data

\begin{tabular}{||l|c||}
\hline \multicolumn{1}{|c|}{ Model Parameter } & Value \\
\hline \hline Initial Water Saturation & 0.47 \\
\hline Density of Dry Clay $\rho_{s} \mathrm{Kg} / \mathrm{m}^{3}$ & 1,800 \\
\hline Initial Porosity of Clay $\phi$ & 0.35 \\
\hline Initial Isotropic Stress in Clay $(\mathrm{MPa})$ & 3.7 \\
\hline Elastic Shear Modulus $G(\mathrm{MPa})$ & 214 \\
\hline Elastic Bulk Modulus $K(\mathrm{MPa})$ & 2,286 \\
\hline Cohesion Parameter $c_{F}(\mathrm{MPa})$ & 1.8 \\
\hline Internal Friction Coefficient $\mu_{F}$ & 0.170 \\
\hline Clay Swelling Expansion Coeff. $\beta$ & 0.0005 \\
\hline
\end{tabular}




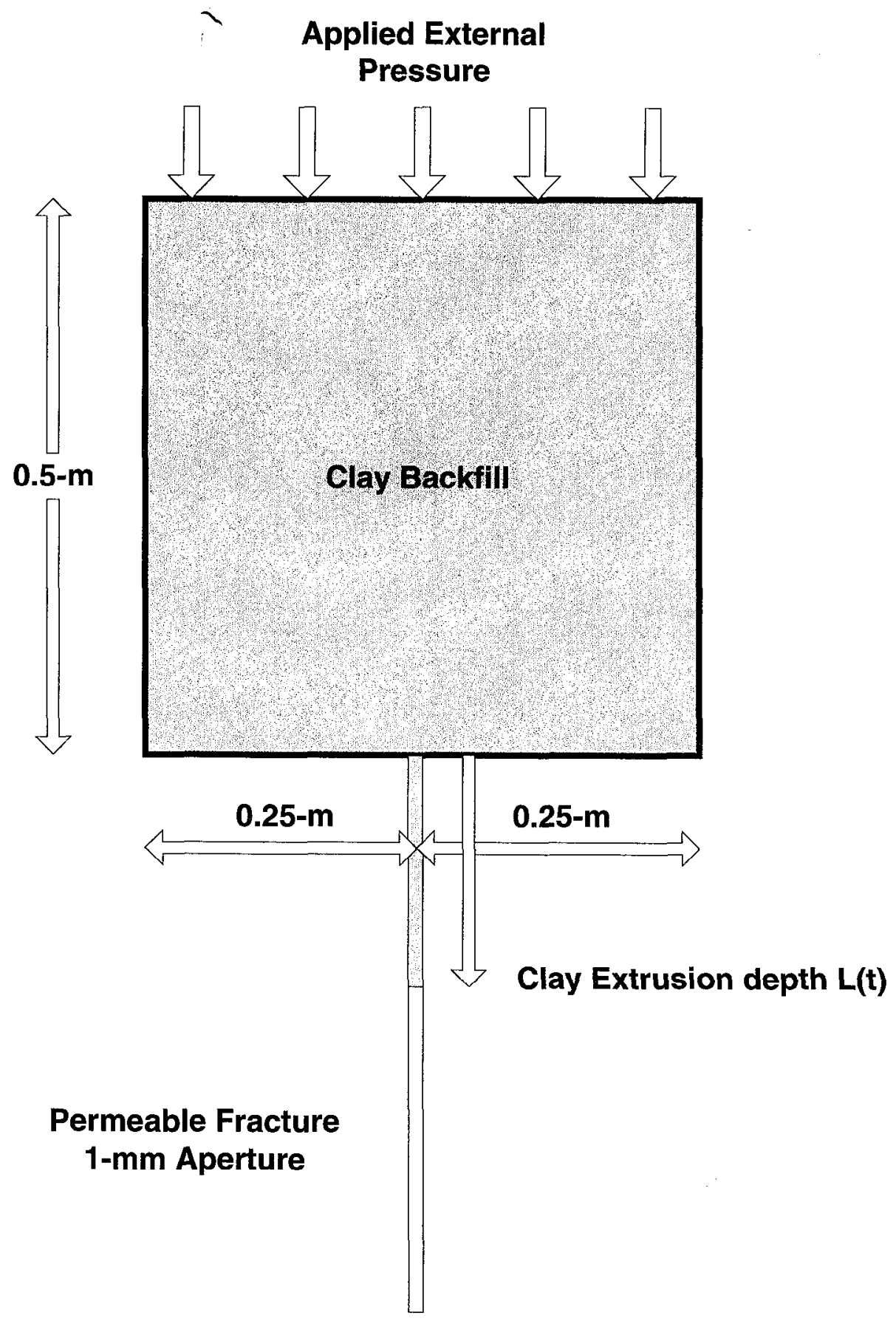

Figure 3.1-1: Physical Configuration for Problem 1 
The clay is assumed to be elasto-plastically deformed and driven into the fracture by a uniform normal stress that is suddenly exerted on the clay backfill at the tunnel roof and maintained constant thereafter. The tangential shear stress at this surface is taken to be zero. The applied normal stress is attributed to failure of the tunnel roof due to external causes and its subsequent settlement onto the clay backfill. In modeling this problem, we assume that the water saturation remains unchanged at its initial value of 0.47 , and that the clay deforms according to the approximate Extended Drucker-Prager Elasto-Plastic model presented previously in Section (2.1.3). We present calculations with the tunnel and fracture surfaces modeled as both frictionless surfaces (Case-1) and as frictional slide-surfaces (Case-2). The input data used for computing Problem 1 is given in Table 3.1-1.

\subsubsection{Computational Results}

The ALE-3D code computed this problem without any difficulty using a time scaling factor equal to $5.0 \times 10^{6}$, which was well within the accuracy and stability limits for the problem. In most locations within the computational domain, the finite-element computational grid generated initially for computing this problem was not further refined, but was automatically restored to its initial configuration at the end of every time step. The exception to this was at the entrance to, and within, the fracture where most of the deformation occured during the computational period. The initial finite-element mesh near (within $0.1 \mathrm{~m}$ ) the entrance to the 1-mm wide fracture is shown in Figure 3.1-2. As the computation proceeded, the grid extended into the fracture as can be seen in Figure 3.1-3. In Figures 3.1-3 and 3.1-4, the clay interface locations at 40,000 secs and 60,000 secs, respectively, are shown for Case-1 in which the tunnel walls and fracture surfaces are treated as frictionless and a $40 \mathrm{MPa}$ external pressure is applied at the roof of the tunnel. Because the fracture surfaces are frictionless, it can be seen that the clay/air interface within the fracture is a flat surface. The contour plot of the average normal stress distribution shown in Figure 3.1-5 for Case-1 at 60,000 seconds shows the stress concentration at the entrance to the fracture. The time evolution of clay penetration into the fracture in Case-1 is shown in Figure 3.1-9 over the computational period of 0 to 60,000 seconds.

The same problem was recomputed as Case- 2 with frictional walls and a much lower applied external stress of $7 \mathrm{MPa}$. The contours of the average normal stress disctribution superimposed on the computational mesh is shown for this Case in Figure 3.1-6 at 7.0 $\times 10^{8}$ seconds. Because of the much lower applied stress and the presence of frictional walls, a much greater time was needed to extrude the clay into the fracture in Case-2 compared to Case-1. Also, as can be scen from Figure 3.1-6, because of friction at the fracture surfaces, the shape of the clay/air interface within the fracture was no longer flat as in Case-1. It formed a cylindrical piston with a round head that preceded the clay plug contacting the walls of the fracture behind it. 
In Figure 3.1-7, the contours (4 to $7 \mathrm{MPa}$ ) of the distribution of the average normal stress within the clay for Case- 2 is shown at $7.0 \times 10^{8}$ seconds over a larger neighborhood of the fracture. Because of the presence of wall friction, the shape of the stress contours are different from those given previously in Figure 3.1-5 for Case-1. The contours $(0$ to 0.05$)$ of plastic strain in the clay in the neighborhood of the fracture are given in Figure 3.1-8. It can be seen from this plot that a cone shaped slip zone forms just above the entrance to the fracture as the clay is extruded into the fracture, while there is little plastic deformation elsewhere within the clay. The piston-like shape of the clay/air interface can also be clearly seen in this Figure. 


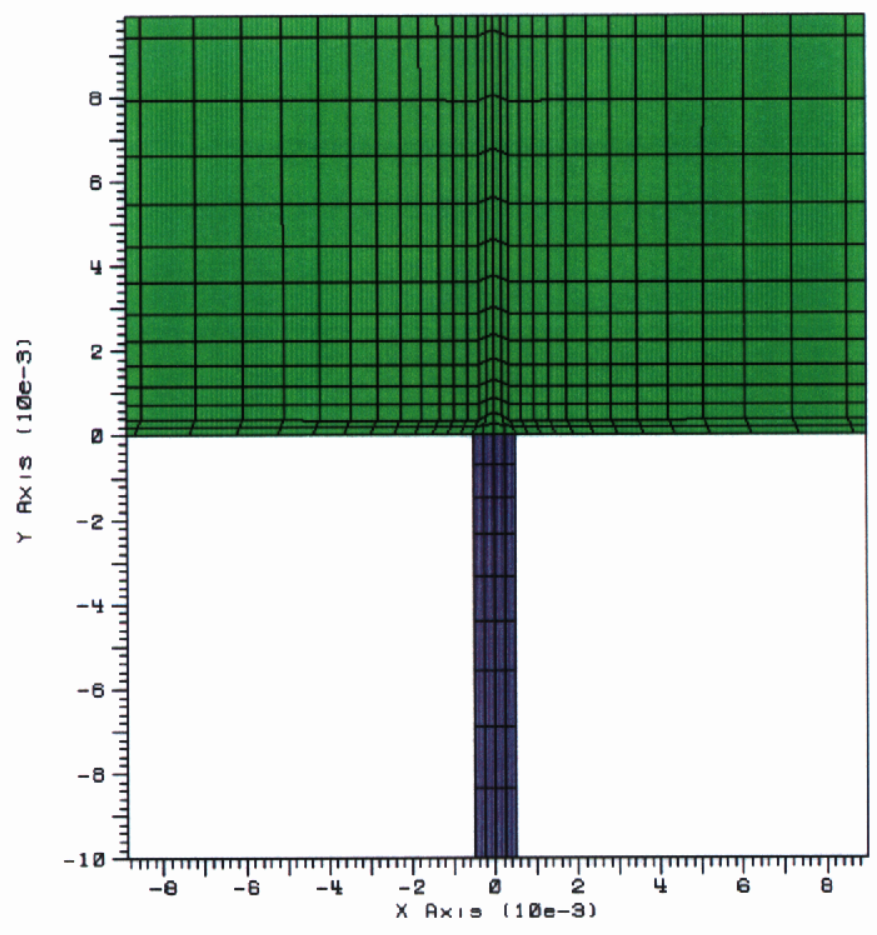

Figure 3.1-2: Initial finite element computational mesh at entrance to fracture (Case-1 and Case-2) 


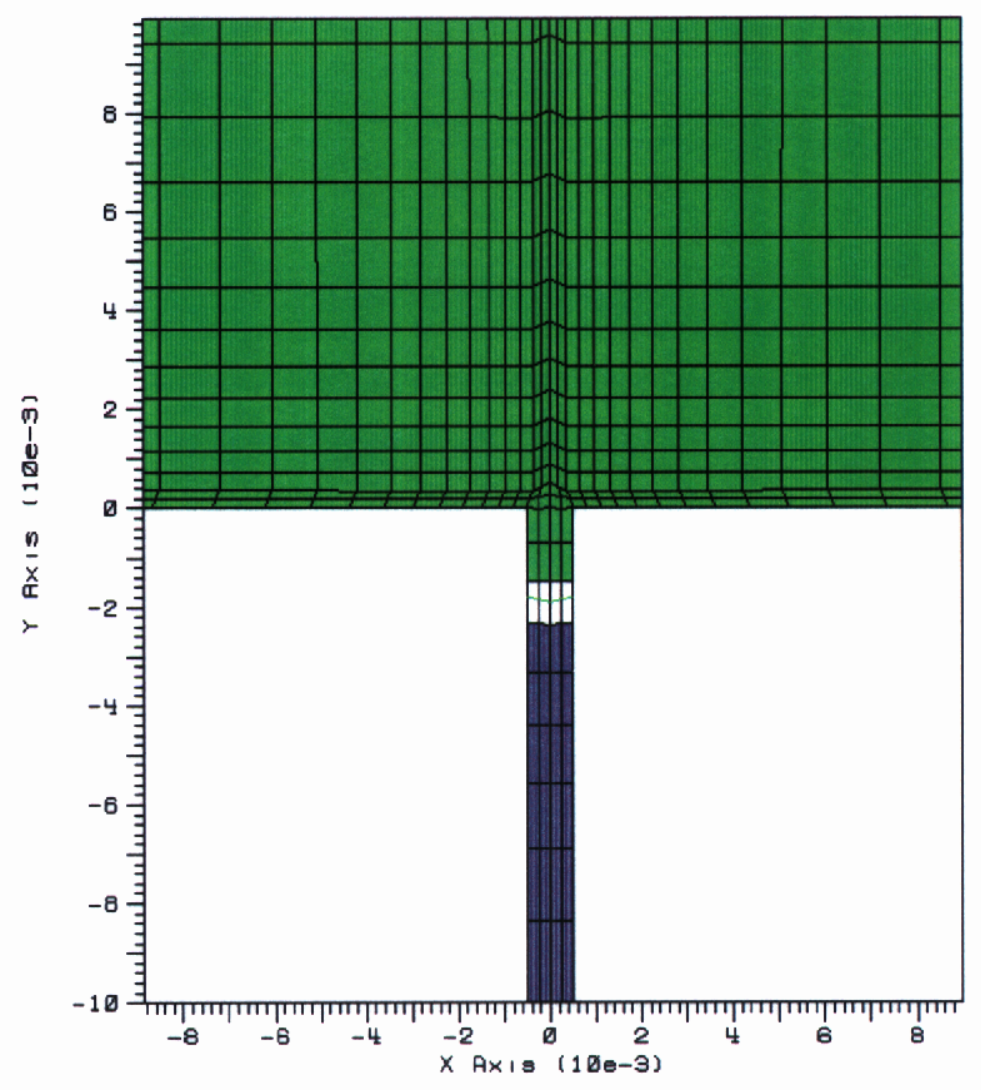

Figure 3.1-3: Mesh plot showing the clay interface location in fracture at 40,000 secs; Case-1: Frictionless walls with $40 \mathrm{MPa}$ applied external stress 


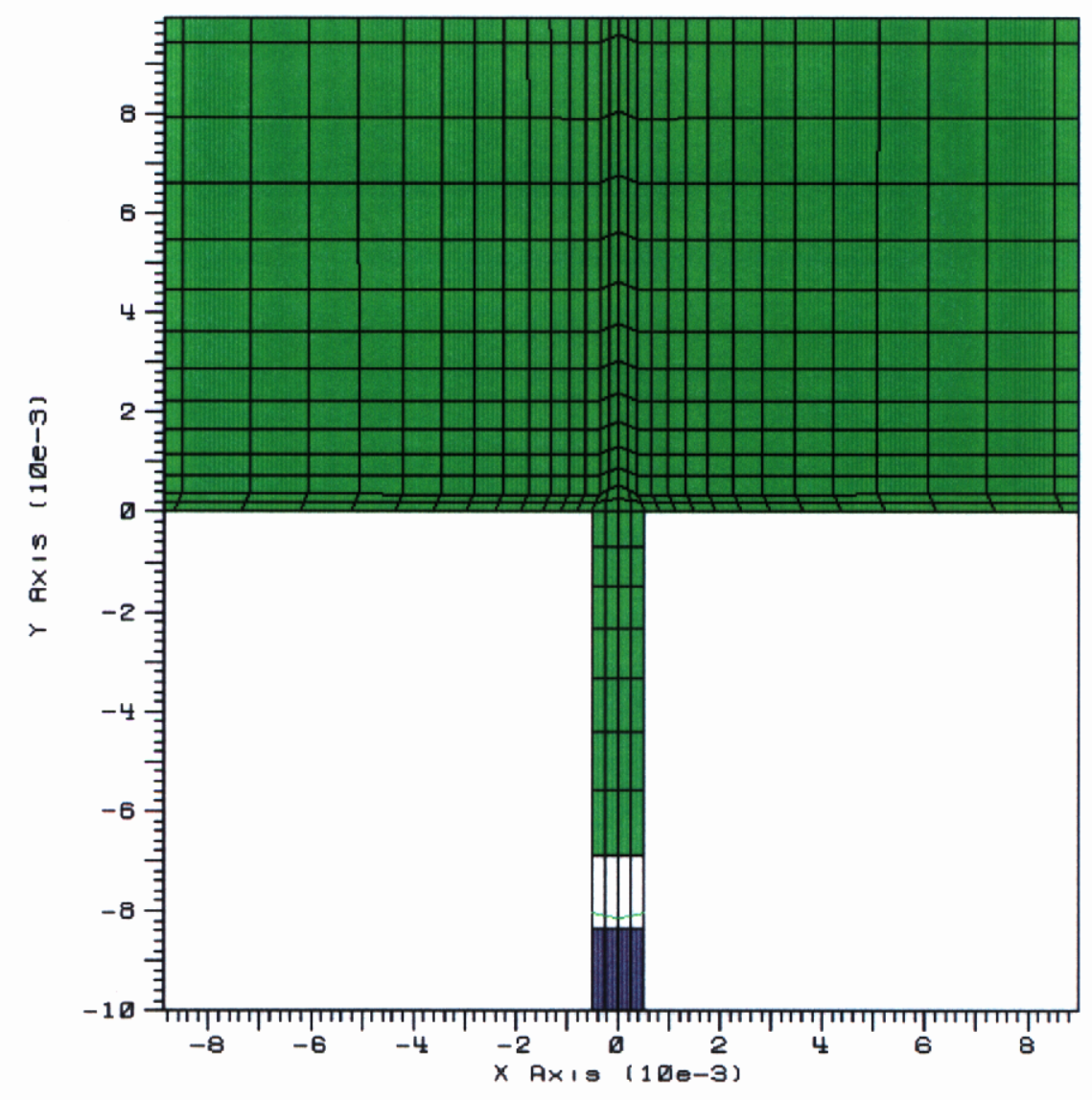

Figure 3.1-4: Mesh plot showing clay interface location in fracture at 60,000 secs; Case-1: Frictionless walls and $40 \mathrm{MPa}$ applied external stress 


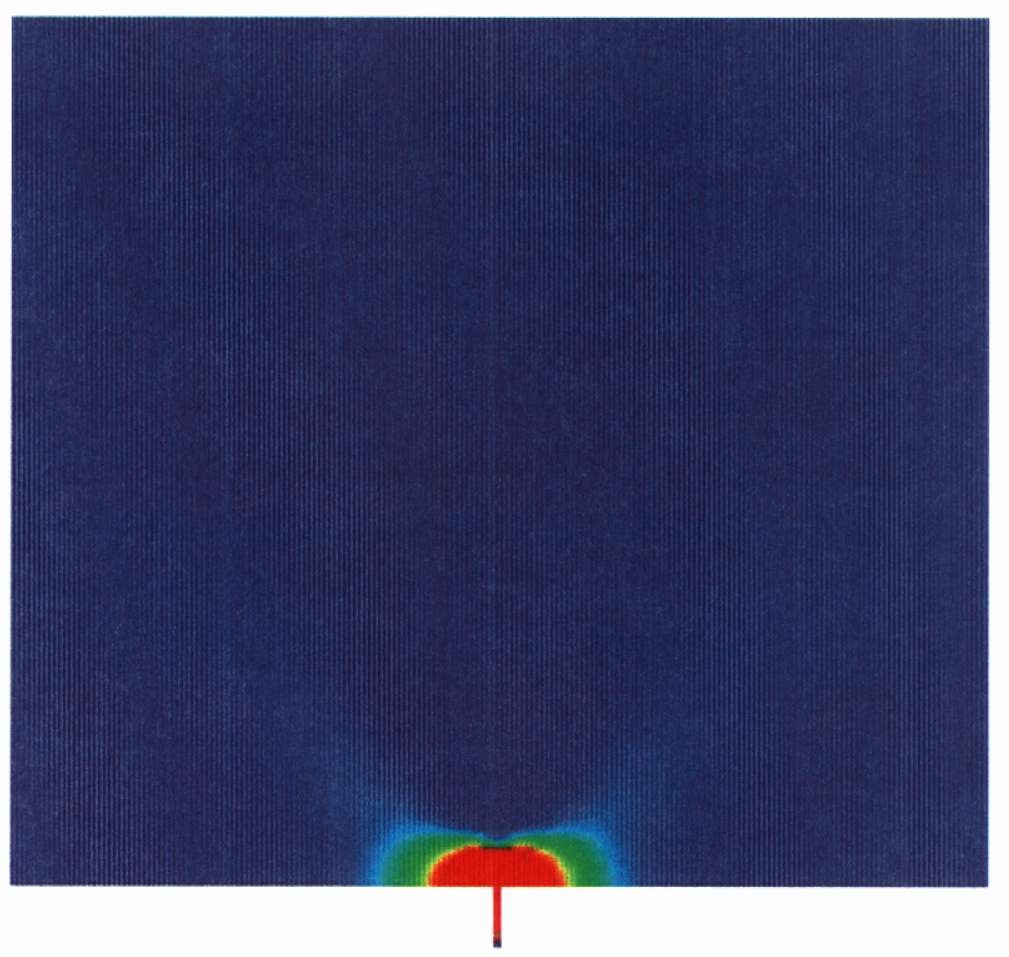

Figure 3.1-5: Contour plot of average normal stress distribution in clay at 60,000 secs showing clay interface location in fracture; Case-1: Frictionless walls and $40 \mathrm{MPa}$ applied external stress 


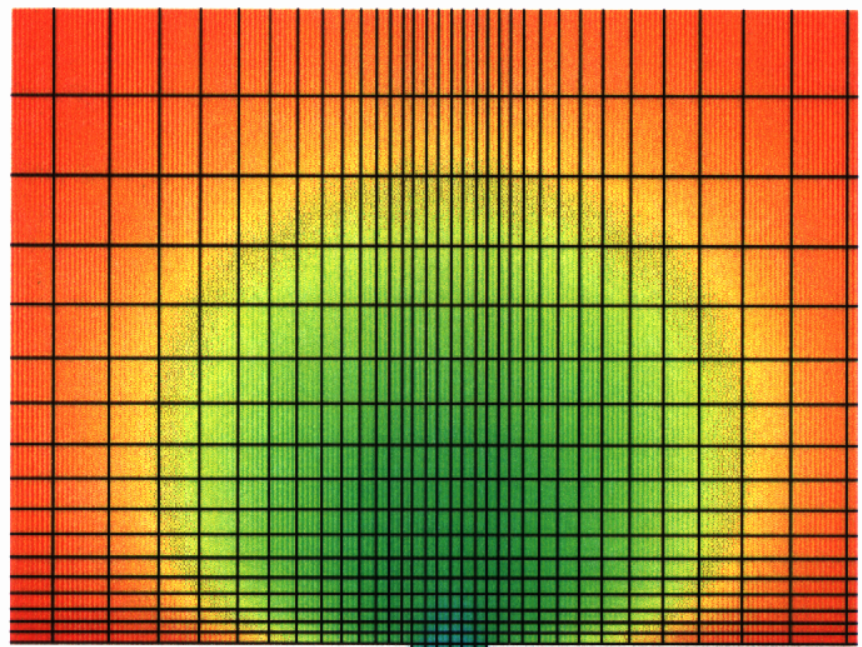

Figure 3.1-6: Mesh plot and contour plot of average normal stress distribution in clay at $7.0 \times 10^{8}$ secs showing clay interface location in fracture;

Case-2: Frictional walls and 7 MPa applied external stress 


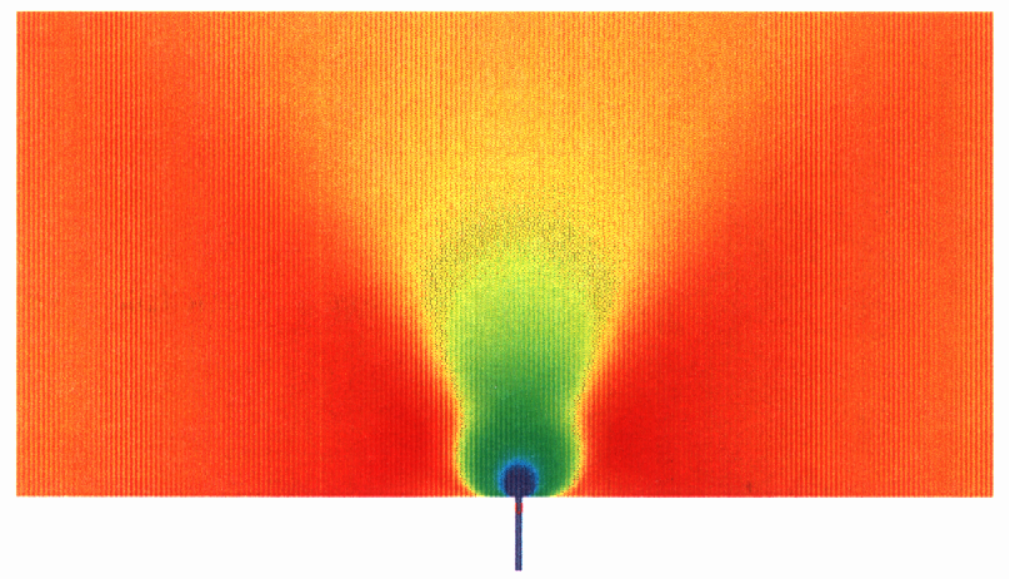

Figure 3.1-7: Contour plot of average normal stress distribution (4 to $7 \mathrm{MPa}$ ) in clay at $7.0 \times 10^{8}$ secs showing clay interface location in fracture;

Case-2: Frictional walls and $7 \mathrm{MPa}$ applied external stress 


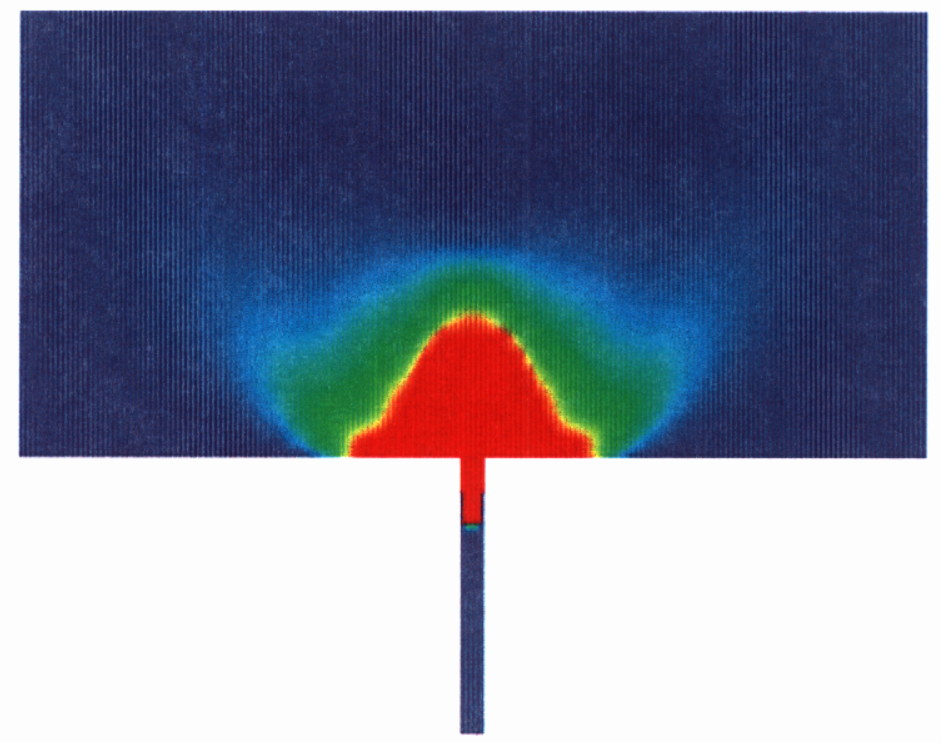

Figure 3.1-8: Contour plot of plastic strain distribution ( 0 to 0.05$)$ in clay at $7.0 \times 10^{8}$ secs showing clay interface location in fracture;

Case-2: Frictional walls and $7 \mathrm{MPa}$ applied external stress 


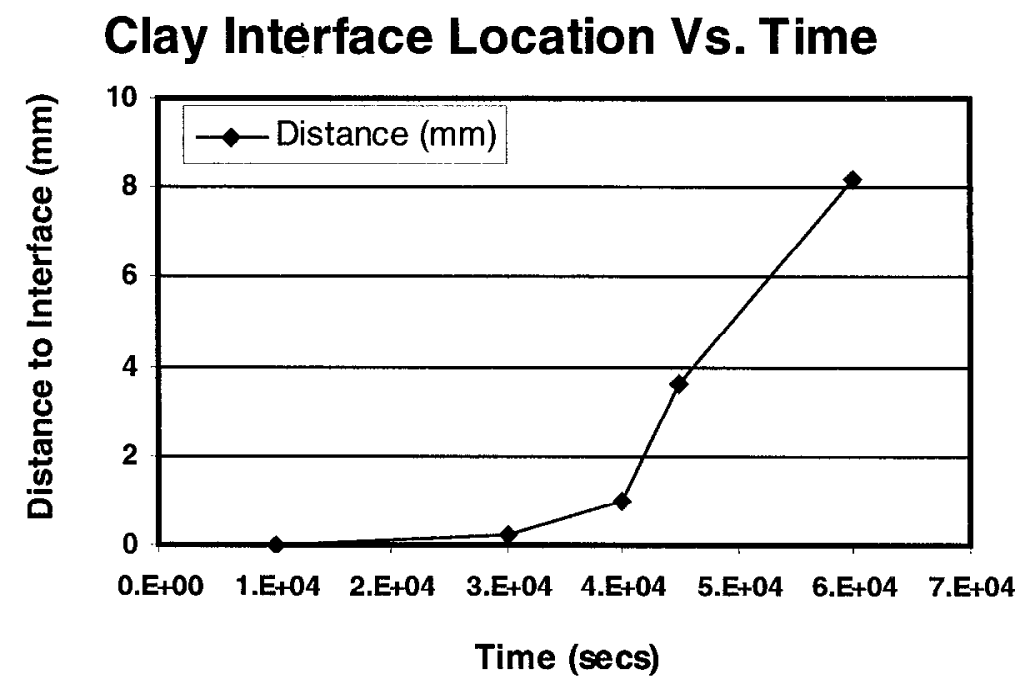

Figure 3.1-9: Clay interface location in fracture as a function of time; Case-1: Frictionless walls and $40 \mathrm{MPa}$ applied external stress 


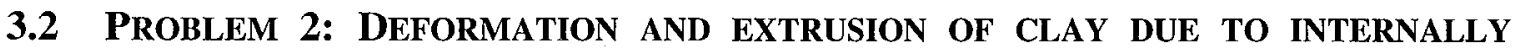 DEVELOPED SWELLING INDUCED PRESSURES}

The objective of Problem 2 is to meet the requirements of sub-Task 4.1 of this project by demonstrating the capability of the ALE-3D code to model clay extrusion into fractures due to the softening and swelling of clay when contacted by water. The water is assumed to gradually infiltrate into the clay from permeable fractures located at the tunnel roof. The goal of this simulation is to demonstrate the ability of the ALE-3D code to simultaneously compute the coupled processes of water diffusion into the unsaturated clay, the development of swollen and softened clay zones behind the wetting front, the elasto-plastic deformation of clay within the tunnel, and the extrusion of clay into the fracture located at the floor of the tunnel.

\subsubsection{Assumptions \& Input Data}

The physical configuration for Problem 2 is shown in Figure 3.2-1. In this problem, the driving force for clay deformation is derived from the swelling pressure developed when the clay is wetted by water that seeps into the clay-backfill from the roof of the tunnel. The water source is modeled as a sudden increase in water saturation at the tunnel roof boundary from 0.47 to a constant uniform value of 1.0. All other tunnel and fracture surfaces are assumed to be impermeable.

Table 3.2-1: Problems 2 \& 3 - Input Model Parameter Data

\begin{tabular}{||l|c|c||}
\hline \multicolumn{1}{|c|}{ Model Parameter } & Value & Value \\
\hline Water Saturation & 0.47 & 0.98 \\
\hline Density of Dry Clay $\rho_{s} \mathrm{Kg} / \mathrm{m}^{3}$ & 1,800 & \\
\hline Initial Porosity of Clay $\phi$ & 0.35 & \\
\hline Initial Isotropic Stress in Clay $(\mathrm{MPa})$ & 3.7 & \\
\hline Elastic Shear Modulus $G(\mathrm{MPa})$ & 214 & 36 \\
\hline Elastic Bulk Modulus $K(\mathrm{MPa})$ & 2,286 & 381 \\
\hline Cohesion Parameter $c_{F}(\mathrm{MPa})$ & 1.8 & 1.0 \\
\hline Clay Swelling Expansion Coeff. $\beta$ & 0.0005 & 0.0005 \\
\hline Watcr Diffusivity in Clay $D_{w}\left(\mathrm{~m}^{2} / \mathrm{sec}\right)$ & $1.0 \times 10^{-9}$ & $1.0 \times 10^{-9}$ \\
\hline
\end{tabular}




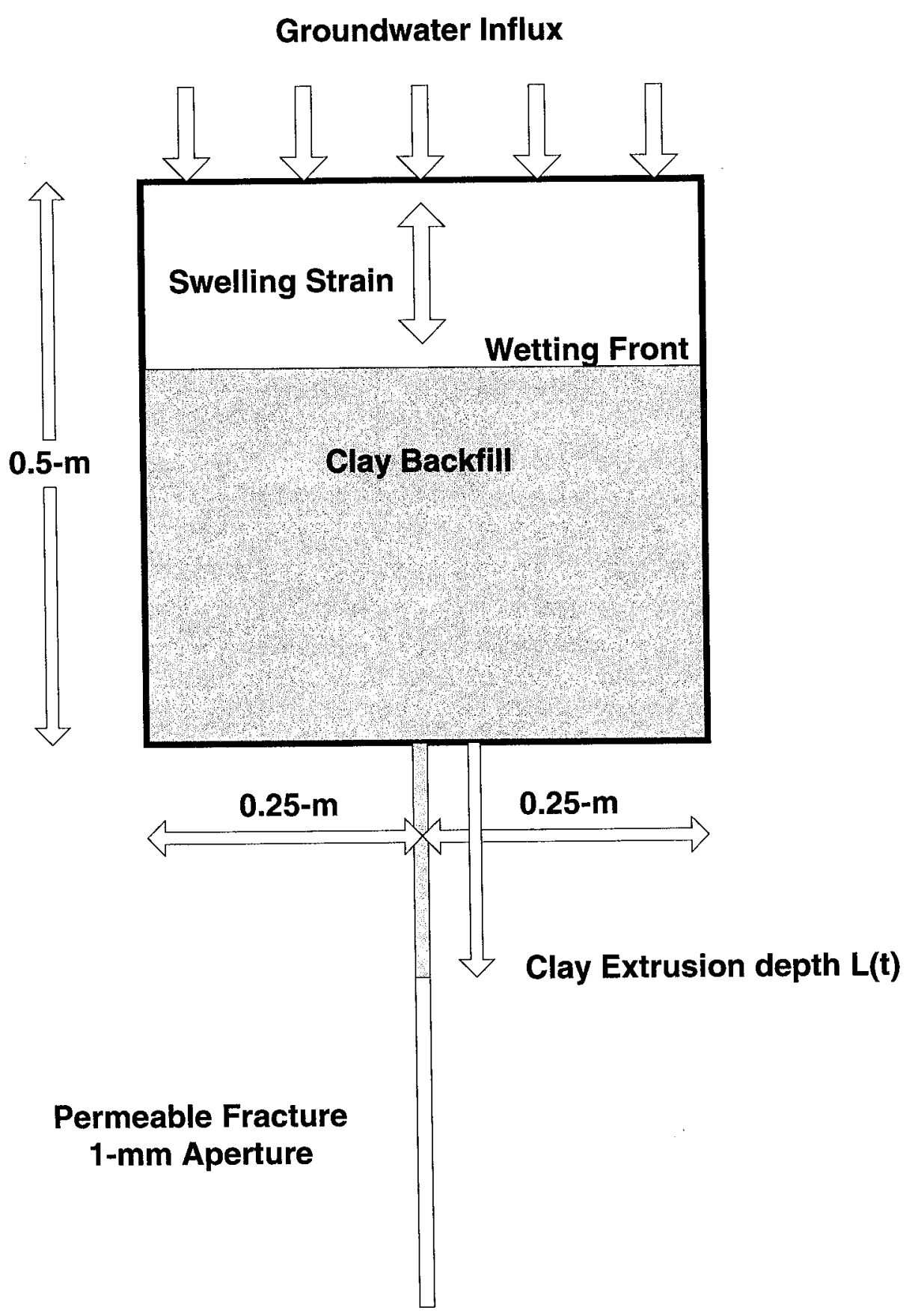

Figure 3.2-1: The Physical Configuration for Problem 2 
The infiltration of water from the wetted roof boundary into the interior of the clay backfill is modeled using the diffusion equation for water saturation given by Equation (2.1-46). In modeling clay deformation, because the ALE-3D code could not allow for a saturation dependent frictional coefficient, we assumed this parameter to be zero in the approximate Extended Drucker-Prager Elasto-Plastic model presented previously in Section (2.1.3) but allowed the cohesion material parameter to decrease linearly with water saturation as given in Table 3.2-1. We present calculations with the tunnel and fracture surfaces modeled as rigid frictional slide-surfaces.

\subsubsection{Computational Results}

In this coupled-processes problem, the driving force was derived from the swelling of the clay when wetted by water that infiltrated into the clay from the tunnel roof. The ALE-3D code computed this problems without any difficulty using a time scaling factor equal to $7.5 \times 10^{10}$ and handled the extreme distortion of the clay at the entry to the fracture and the movement of a wetting front into the clay.

The contours ( 1 to $1.35 \mathrm{MPa}$ ) of the yield strength of the clay, are shown in Figure 3.2-2 at $4.0 \times 10^{8}$ seconds after water saturation at the tunnel roof rises to from 0.47 to 1.0 . This Figure shows the yield strength of clay decreasing from its initial value of $1.35 \mathrm{MPa}$ at the floor of the tunnel (red color) to a value of $1 \mathrm{MPa}$ (green color) at the roof of the tunnel due to softening upon contact with water. The water saturation decreases in the downward direction from full saturation (1.0) to its initial value of 0.47 at the floor of the tunnel. Figure 3.2-3 shows a magnified view of the contours $(0$ to 0.05$)$ of plastic strain at the entrance to the fracture. It also shows the clay plug that is extruded into the fracture due to stress developed by swelling of the clay. Because of frictional drag at the fracture surfaces, the clay/air interface has the piston-like round-headed cylindrical shape previously seen in Case-2 of Problem 1. In Figure 3.2-4, a contour plot (0 to $3.5 \times 10^{-12}$ $\mathrm{m} / \mathrm{sec}$ ) of the magnitude of the flow speed of the clay is shown. As can be expected, the flow of clay is concentrated in the immediate vicinity of the fracture where the driving stresses are relieved by the motion of the clay into the fracture. 


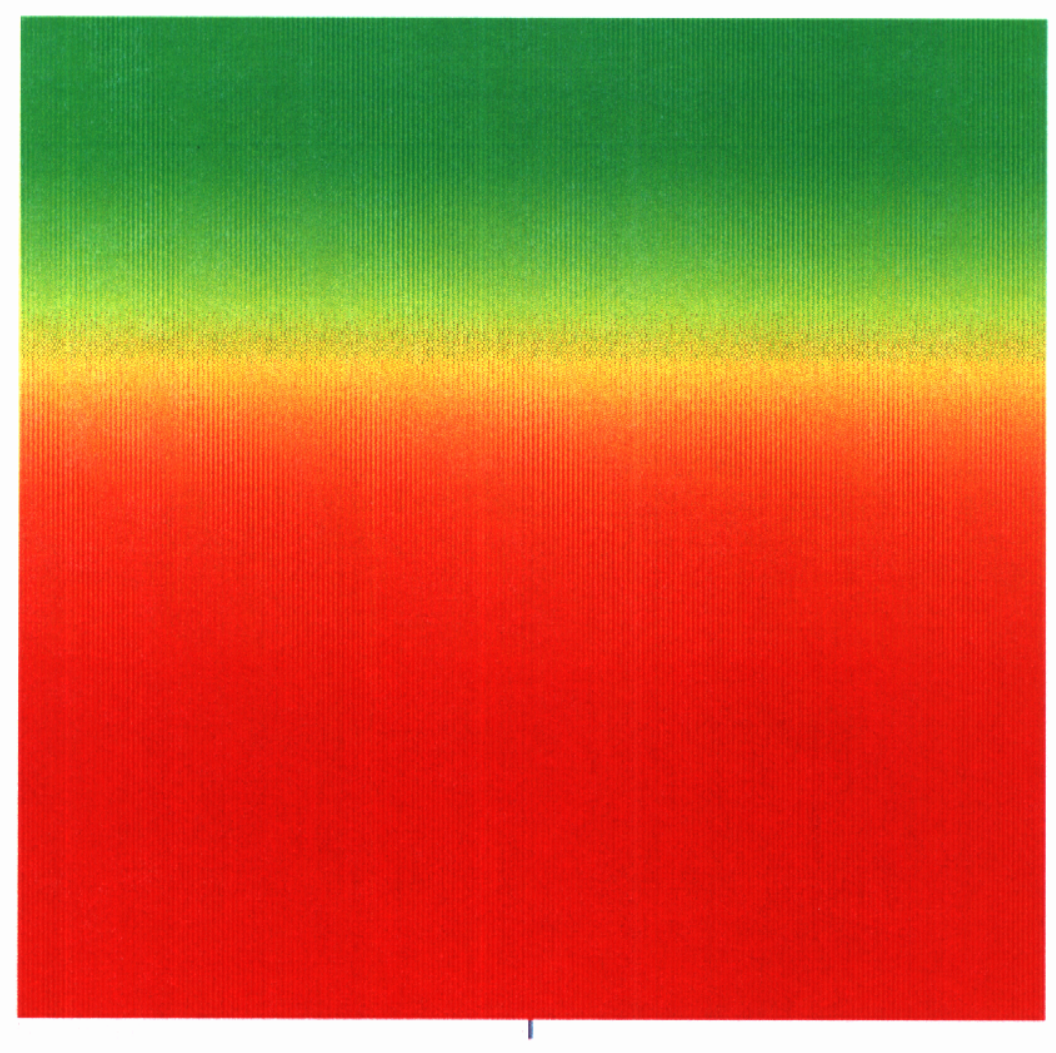

Figure 3.2-2: Contour plot of yield strength distribution (1 to $1.35 \mathrm{MPa}$ ) in clay at $4.0 \times 10^{8}$ secs 


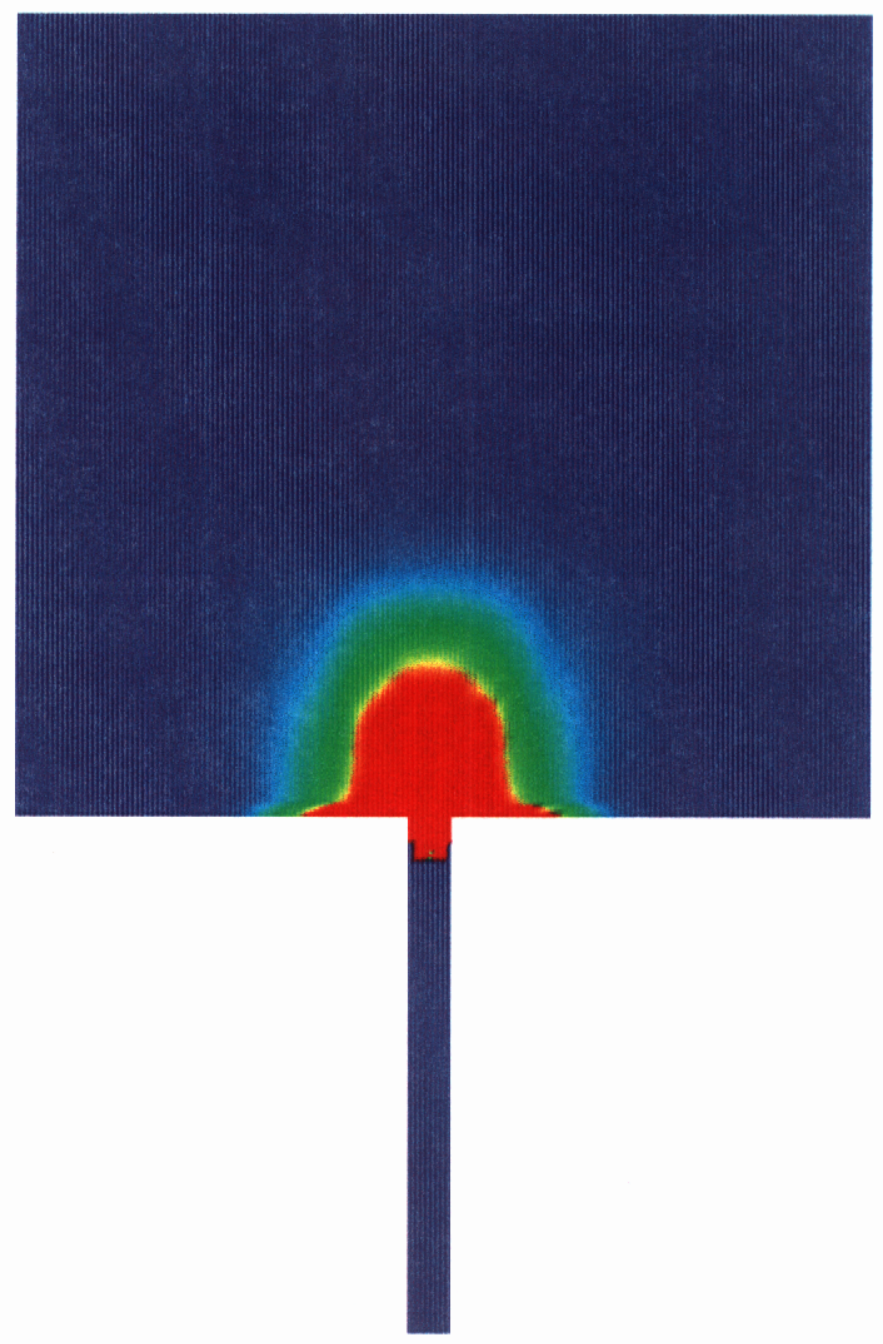

Figure 3.2-3: Contour plot of plastic strain distribution ( 0 to 0.05 ) in clay at $4.0 \times 10^{8}$ secs 


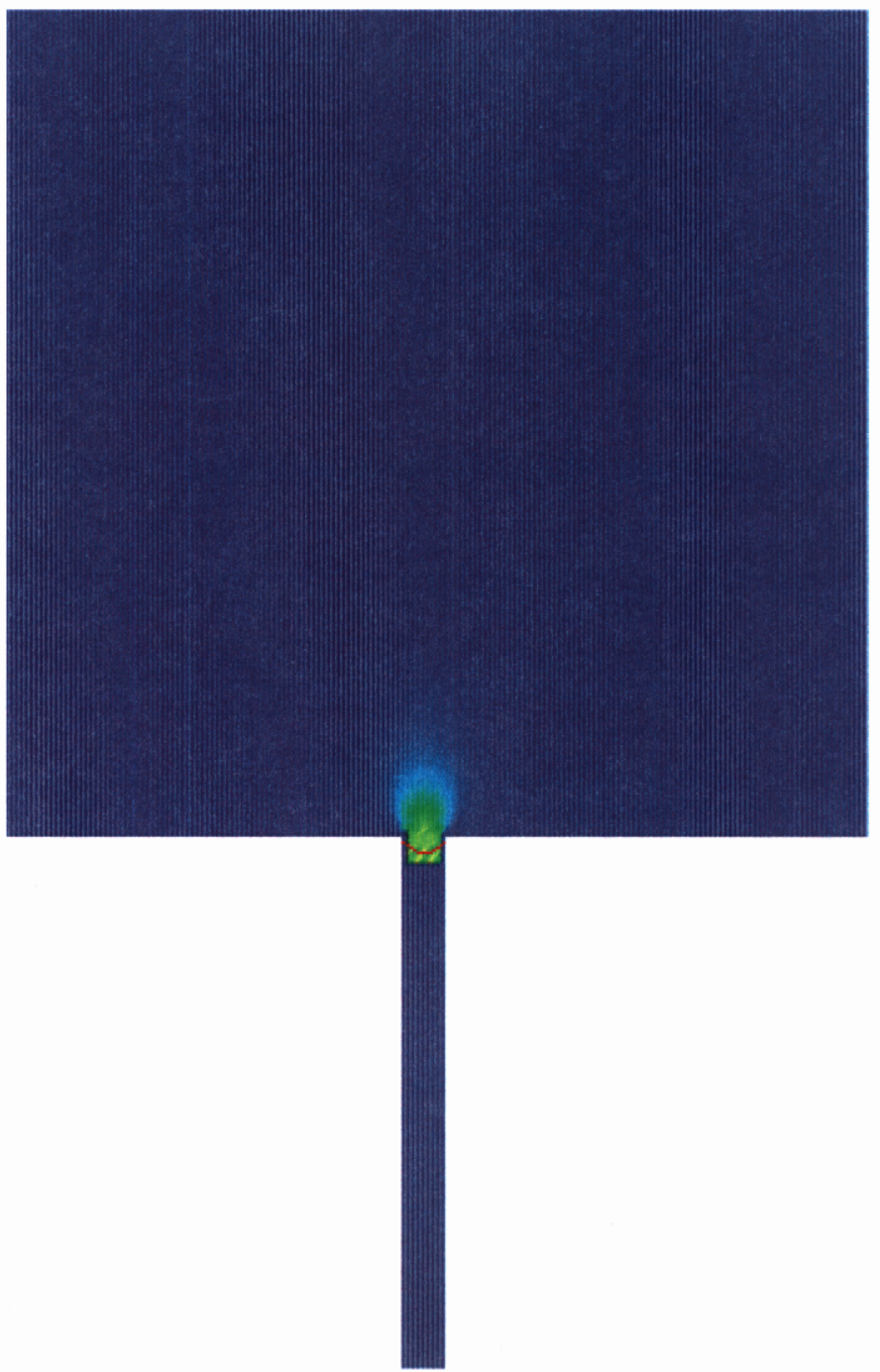

Figure 3.2-4: Contour plot of flow velocity magnitude distribution $\left(0\right.$ to $3.5 \times 10^{-12} \mathrm{~m} / \mathrm{sec}$ ) in clay at $4.0 \times 10^{8}$ secs. 


\subsection{Problem 3: Displacement of an overPack EMBedded in Clay due to GRAVITATIONAL FORCES AND SWELLING INDUCED PRESSURES}

The objective of Problem 3 is to meet the requirements of sub-Task 4.2 of this project by demonstrating the capability of the ALE-3D code to model more realistic problems that involve the migration of steel overpacks emplaced in a clay-filled tunnels when the clay properties are altered by contact with infiltrating water. The downward migration of the steel overpack in this problem is due to elasto-plastic flow of clay around the overpack. In this problem, the fracture was not included and clay extrusion into the fracture was not modeled. The driving forces for the elasto-plastic flow are the stresses exerted on the clay by the weight of the overpack and the stress increase above the overpack due to the swelling of clay upon contact with water infiltrating inwards from the tunnel roof.

\subsubsection{Assumptions \& Input Data}

This Problem was modeled in two spatial dimensions as shown in Figure 3.3-1 with a $0.2-\mathrm{m}$ wide $\mathrm{x}$ 0.4-m tall rigid-walled steel overpack located at the center of a $0.5-\mathrm{m}$ wide $x$ $0.5-\mathrm{m}$ tall clay-filled rigid-walled tunnel of rectangular cross-section. The weight of the steel overpack was taken to be $1,000 \mathrm{Kg}$ per meter of clay thickness normal to the cross-section of the tunnel. Water is assumed to infiltrate inwards into the clay-backfill from the tunnel roof. The water source at the tunnel roof and the flow of water within the clay are modeled as in Probem 2. Furthermore, the clay is assumed to deform according to the approximate Extended Drucker-Prager Elasto-Plastic model presented previously in Section (2.1.3) with elasto-plastic material parameters that decrease linearly with water saturation as given in Table 3.2-1. Frictional interfaces were used along the tunnel walls and the overpack surface.

\subsubsection{Computational Results}

The ALE-3D code computed this problem also without any difficulty using a time scaling factor equal to $7.5 \times 10^{10}$, which was well within the accuracy and stability limits for the problem. The finite-element computational grid generated initially for computing this problem, shown in Figure 3.3-2, was not further refined, but was automatically restored from its deformed configuration to its initial configuration at the end of every time step. In Figure 3.3-3, a vector plot of clay deformation velocity ( 0 to $\left.9.8 \times 10^{-8} \mathrm{~m} / \mathrm{sec}\right)$ is shown at $9.1 \times 10^{8}$ seconds into the computation. As can be seen from this Figure, the clay was squeezed out from under the overpack and flowed around it towards the roof of the tunnel. The displacement of the overpack varied from no settlement to some settlement as the yield strength was reduced over a practical range. As shown in Figure $3.3-4$, the settlement of the overpack accelerated with time as the water infiltrating into the tunnel softened and reduced the yield strength of the clay. The frictional shear stress along the sides of the overpack proved to be a significant factor that retarded the downward movement of the overpack. 


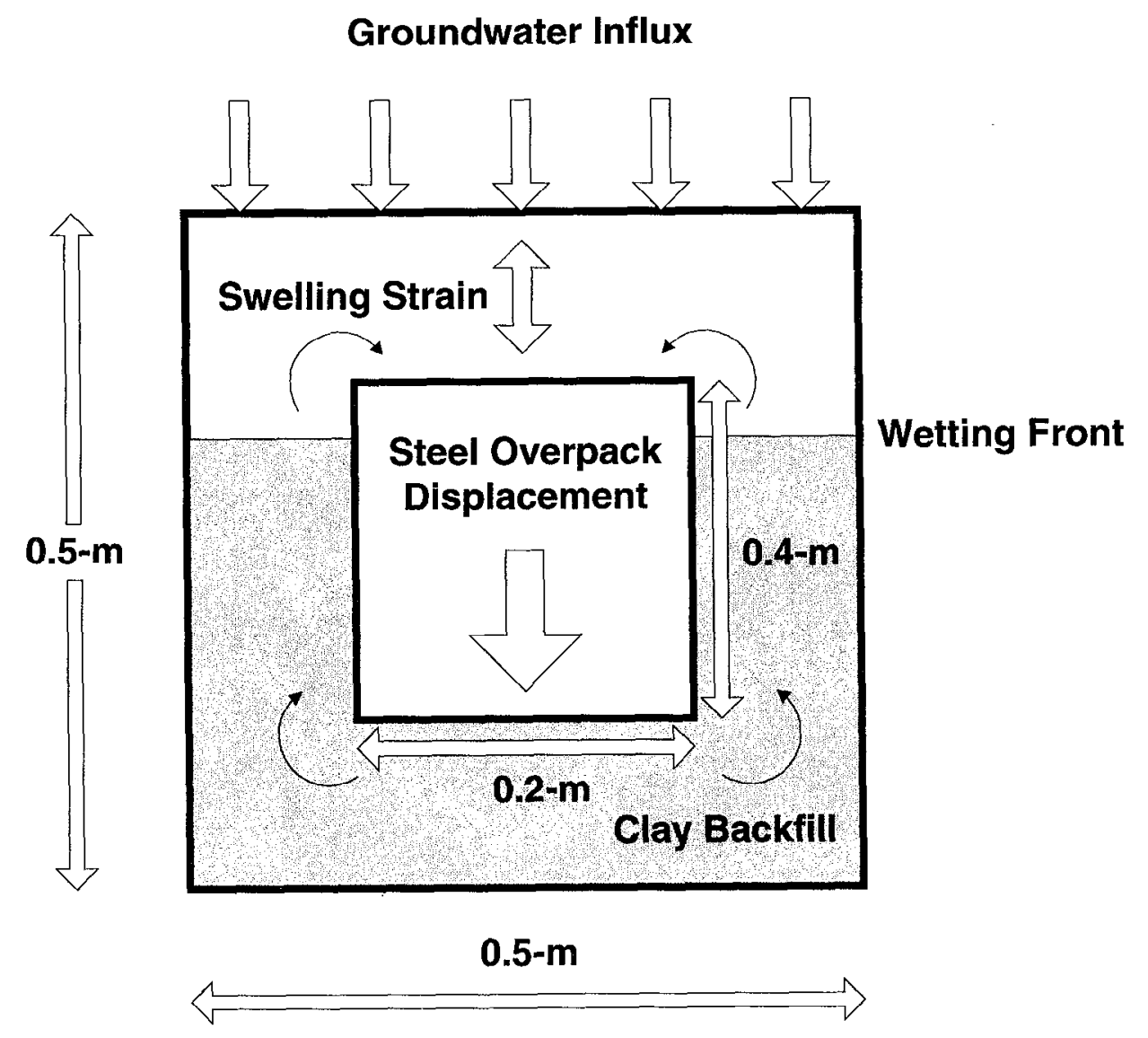

Figure 3.3-1: The Physical Configuration for Problem 3 


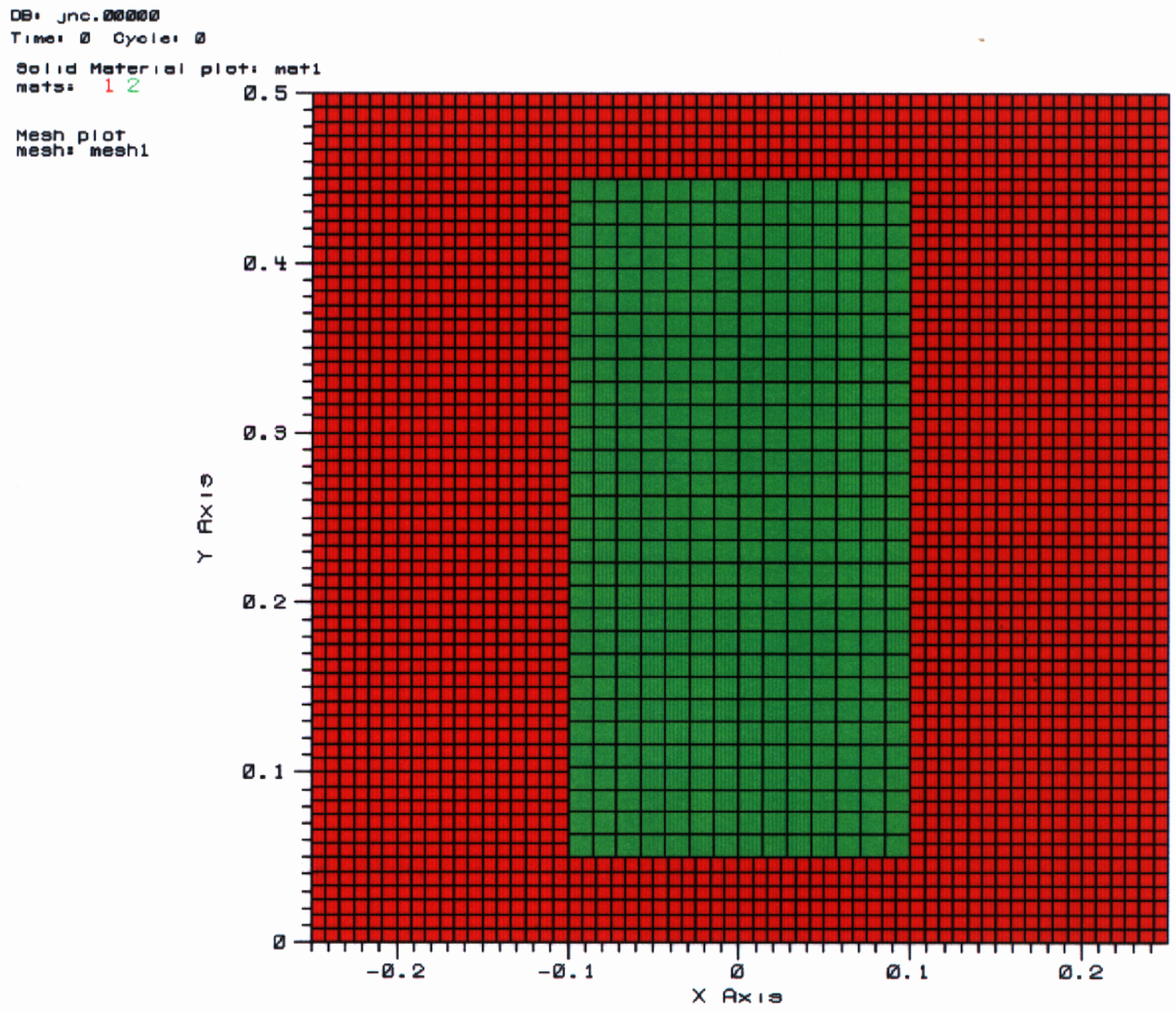

voer Boouoh
Tue Mor $211.19,101999$

Figure 3.3-2: Finite-element mesh for computing overpack displacement 
D8. Jnc.

Time, 9.

Vector plot

v-min: g:

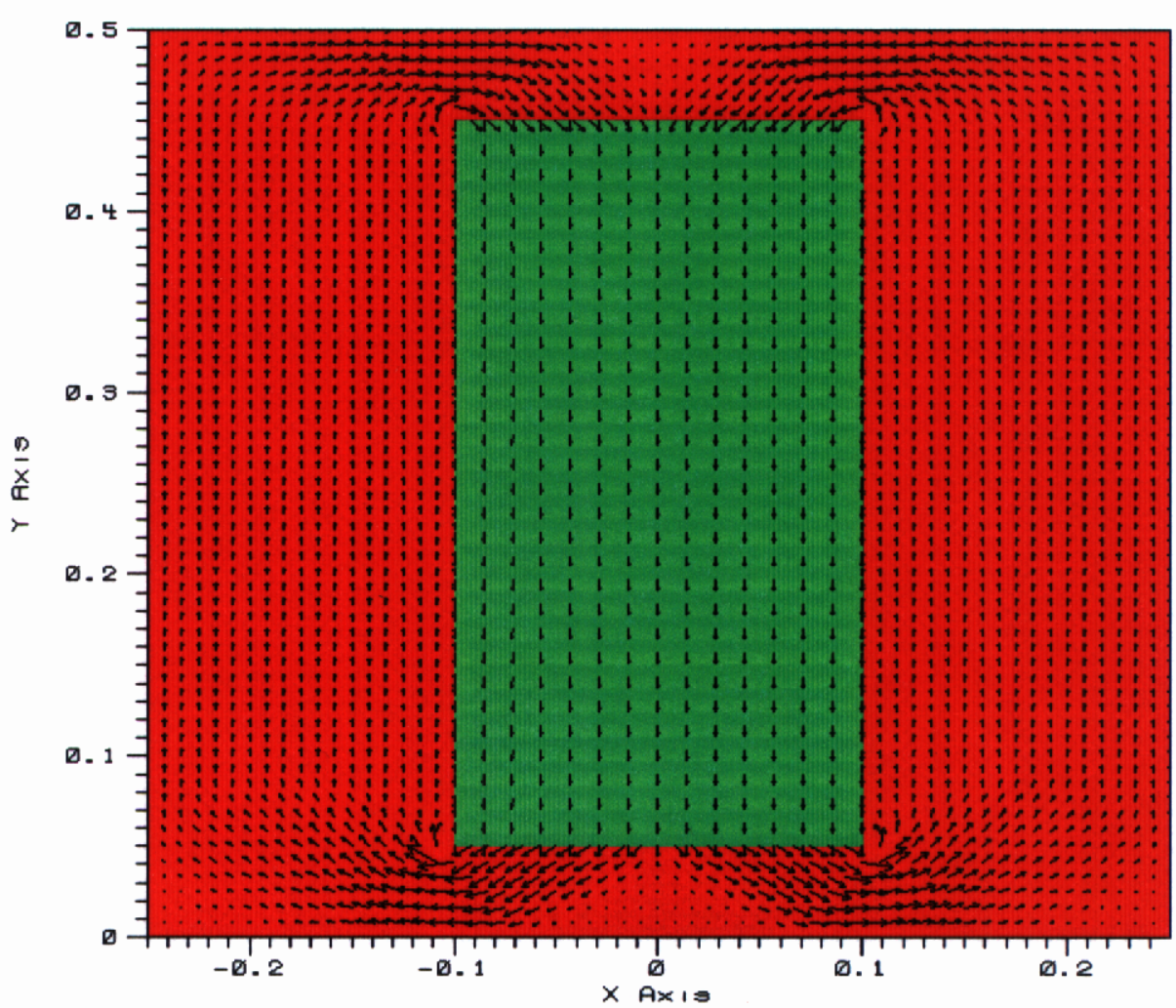

solid material pioti nati

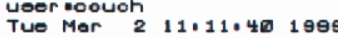

Figure 3.3-3: Clay deformation velocity vector distribution $\left(0\right.$ to $\left.9.8 \times 10^{-8} \mathrm{~m} / \mathrm{sec}\right)$ at $9.1 \times 10^{8}$ secs 


\section{Overpack Displacement Vs Time}

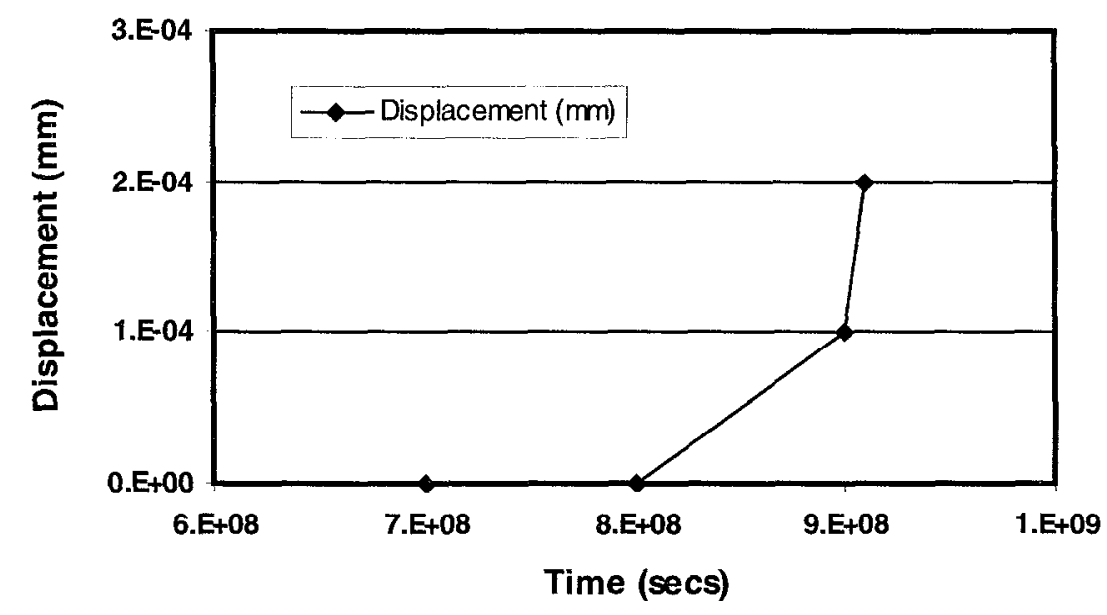

Figure 3.3-4: Downward displacement of overpack as a function of time. 


\section{CONCLUSIONS \& RECOMMENDATIONS}

\subsection{CONCLUSIONS}

We have completed the basic requirements of sub-Task 4.1: Demonstration of NIKE-2D/ALE-3D Mesh Adaptation Capability, and have demonstrated that the ALE-3D code is capable of modeling clay extrusion into fractures, by successfully computing an uncoupled-process problem (Problem 1) and a coupled-process problem (Problem 2). In both problems, a single vertical fracture, 1-mm wide, was located at the center of the floor of a $0.5-\mathrm{m}$ wide $\times 0.5-\mathrm{m}$ tall rigid-walled clay-filled tunnel. In the first uncoupledprocesses problem, the driving force for clay extrusion was a uniform pressure applied to the clay at the roof of the tunnel. In the second coupled-processes problem, the driving force was derived from the swelling of the clay when wetted by water that infiltrated into the clay from the tunnel roof. We treated the tunnel and the fracture as rigid and used estimated material properties for the clay. The ALE-3D code computed these two problems without any difficulty with explicit time stepping methodology by using mass scaling to allow the use of large time steps. It handled both the extreme distortion of the clay at the entry to the fracture and the movement of a wetting front into the clay over a time scale of years. However, no independent verification of the accuracy of the computed results was made due to limitations in time and funds.

We also met the basic requirements of sub-Task 4.2, by successfully computing a more realistic problem (Problem 3) of the downward migration of a steel overpack emplaced in a clay-filled tunnel. The downward settlement was due to elasto-plastic flow of clay around the overpack driven by the weight of the overpack, the stress increase due to clay swelling above the overpack due to water influx at the tunnel roof and the wetting induced softening of clay that supported the steel overpack. Frictional interfaces were used along the tunnel walls and the overpack surface. Displacement of the overpack varied from no settlement to some settlement as the yield strength of the clay was reduced over a practical range. The frictional shear stress along the sides of the overpack was a significant factor that retarded the downward movement of the overpack. As in the case of sub-Task 4.1, no independent verification of the accuracy of the computed results was made due to limitations in time and funds.

\subsubsection{Model Applicability}

We developed a rigorous theoretical model of the coupled processes that govern water transport within a porous elasto-plastically deforming clay material subject to swelling. By examining the approximations necessary to reduce this rigorous formulation to form of the governing equations solved in the ALE-3D code, we identified the assumptions that must be made to utilize the existing ALE-3D code to compute the problems of interest to this Task. The most important of these approximations are:

1. The clay behaves as a porous deformable material satisfying an effective stress law for partitioning the total stress between the solid and fluid phases, 
2. The constitutive behavior of effective solid stress for clay satisfies an associated Drucker-Prager type frictional elasto-plastic flow model extended to incorporate isotropic swelling of clay and water saturation dependent plasticity parameters,

3. The transport of water within the clay under unsaturated conditions can be described in terms of relative permeability and capillary pressure relationships,

4. The pressure of the air phase within the pores remains essentially constant.

Under these assumptions, the governing equations were reduced to two coupled field equations. The first equation governs the deformation of the clay and has the form of a thermo-elasto-plasto-dynamic equation when water saturation is identified with temperature. The second equation has the form of a heat diffusion equation when water saturation is again replaced by temperature. Consequently, the coupled heat conduction and thermo-elasto-plasto-dynamic deformation modelling capabilities of ALE-3D can be adapted to compute the problems of coupled hydro-elasto-plastic deformation and water transport of interest to this Task. We believe all of these approximations are reasonable and valid in this application.

However, in computing Problems 2 and 3 we werc constrained by some limitations of the ALE-3D code. We could not use the full extended Drucker Prager model for two reasons: 1) some of the terms arising from the dependence of the plastic flow rule on changes in water saturation (as identified in Chapter 2) were not represented in the ALE-3D code, and 2) the dependence on water saturation of the Drucker-Prager model parameter for the internal friction of the clay was not available in the ALE-3D code. For this reason, we used a simple only water saturation dependent yield stress model (i.e., water saturation dependent cohesion with the frictional parameter equal to zero). However, these features can be incorporated in the ALE-3D code in the future with a modest effort.

Furthermore, water transport was modelled approximately in the ALE-3D code via a non-linear diffusion equation in contrast to the full set of convection-diffusion transport equations with capillary and relative permeability constitutive relationships that govern water transport. Although it was not an invalid approximation in the class of problems solved here, it would be more general and more accurate to avoid the assumption of constant air pressure, and to independently solve the equations for water and air transport for one fluid phase pressure and the water saturation.

\subsubsection{Computational Methodology}

The problems addressed here are essentially quasi-static processes that are slow compared to the time of transit of an elastic wave through the clay, and therefore, usually require calculation-intensive implicit time stepping strategies to enable large time steps to be taken. The ALE-3D code, on the other hand, is designed to solve dynamic problems over time periods that are comparable to the wave transit time, and therefore utilizes relatively simple but accurate explicit time stepping strategies based on integrating the inertial acceleration term in time. To enable the ALE-3D code to compute our quasi-static 
problem with large time steps and explicit time stepping, we used the ALE-3D code option of scaling the mass and time in the inertia term. In this way, we were able to compute all three problems with a reasonable amount of computational effort.

Although the ALE-3D code had certain deficiencies in its constitutive models for our application, the great advantage it offered in coping with large deformations and moving material interfaces without manual intervention to regenerate and/or refine the computational grid, was invaluable. This feature yielded very large savings in labor and computational time and enabled these preliminary calculations to be completed within a relatively short time period.

\subsection{RECOMMENDATIONS}

\subsubsection{Model Formulation \& Code Enhancements}

We recommend continued use of the ALE-3D code for the applications in this Task and the implementation of certain code enhancements to adapt and improve the constitutive models incorporated in the code in Task 4.3.

First, we propose that the full extended Drucker-Prager frictional elasto-plastic model with allowance for clay swelling and water saturation dependent parameters, that is presented in this report, be implemented in the code and tested against other codes and available experimental data.

Second, we propose that the work be extended to include an evaluation of both the Cam Clay [8] and the Sandler-DiMaggio Moving Cap [7] elasto-plastic model types and the better of these two types of frictional elasto-plastic models be incorporated in the ALE-3D code as an alternative to the Extended Drucker-Prager model presented in this report. Without further investigation and studies based on measured material property data on clays, it is not possible to predict which of these different types of elasto-plastic models would be better for use in the current application.

\subsubsection{Computations}

We have demonstrated the ability of the ALE-3D code to handle clay extrusion into fractures, the principal issue of interest to the JNC. However, although this work has demonstrated that the ALE-3D code can handle the extremely large material distortion associated with clay extrusion into fractures, and can compute overpack migration problems involving coupled processes of plastic deformation of clay and water infiltration, the calculations were perfomed with roughly estimated clay material properties. Also, the calculations assumed a rigid rock mass and non-deformable fractures, thus possibly underestimating the impact of clay extrusion into fractures. Therefore, the results must be verified using more appropriate material property experimental data and checked against other calculations or direct measurements of the extent of overpack displacement and clay extrusion into fractures. This could not be done 
due to limitations in the available funds, project time and material property data. No conclusion on the significance of impact of clay extrusion into fractures on overpack migration can be made at this time on the basis of these limited calculations; that must await the results of the more detailed parametric study that will be undertaken in subTasks 4.3 and 4.4.

Upon completion of the above two code enhancements, the codes should be verified against other codes, published solutions and/or available experimental data. Then Tasks 4.1 and 4.2 should be repeated and the results compared. The calculations should be extended to include deformation of the tunnel and the fractures, and if feasible, adopt more realistic tunnel/emplacement/overpack geometries and material properties relevant to, and derived from, JNC's on-going applications/experimental program. The ALE-3D code is well suited for such computations. Enhancement of the ALE-3D code to eliminate the deficiencies we have identified here is much less difficult than embedding ALE capabilities within a purely Lagrangian deformation/fluid transport code.

Modeling work under Task 4.4 geared to estimation of the timing, the spatial distribution of rewetting, and overpack migration in the tunnel should be undertaken as originally proposed for this portion of this Task.

\subsubsection{Supporting Experiments}

In addition to bench-scale experiments performed to determine the material properties of the backfill materials, it would be useful to undertake a field-scale experiment to verify and demonstrate that the predictions made with the analysis codes are in fact realistic under as-emplaced repository conditions. For this purpose, we recommend that a field scale experiment be designed and executed in a fractured rock mass, and the measurements be compared against both pre-test predictive analyses and post-test performance analyses. 


\section{REFERENCES}

1. Japan Nuclear Cycle Development Institute [JNC], Supporting Report 2, Repository Design and Engineering Technology, H12 Project for Assessment of the Feasibility of HLW Disposal in Japan, Second Draft, March 29, 1999.

2. Fujita, T., Hara, K., Yuba, Y., and N. Sasaki, Application of Elasto-Plastic Model to Mechanical and Hydraulic Behavior of Buffer Material Under Water Uptake in a Repository, Mat. Res. Soc. Symp.. Proc., Vol. 212, 1991, pp 459-465.

3. Anderson, S., Dube, E., Futral, S., Otero, I., and R. Sharp, Users Manual for ALE-3D, An Arbitrary Langrangian/Eulerian 3D Code System, Lawrence Livermore National Laboratory, March 29, 1996.

4. Couch, R., McCallen, R., Otero, I., and R. Sharp, 3D Metal forming applications of ALE Techniques, Simulation of Materials Processing: Theory, Methods and Applications, Shen \& Dawson (eds), Balkema, Rotterdam, 1995.

5. Drucker, D.C., and W. Prager, Soil Mechanics and Plastic Analysis or Limit Design, Quart. Appl. Math., Vol. 10, No. 2, 1952, pp 157-165.

6. Sandler, I.S., DiMaggio, F.L., and G.Y. Baladi, Generalized Cap Model for Geologic Materials, J. Geotech. Eng.Div., ASCE, Vol. 102, No. GT7, July 1976, pp 683-699.

7. Steinberg, D.J., Cochran, S.G., and Guinan, M.W., A Constitutive Model for Metals Applicable at High-Strain Rate, J. Appl. Phys., Vol. 51, 1980, p. 1498.

8. Schofield, A.N., and C.P. Wroth, Critical State Soil Mechanics, McGraw-Hill Book Company, London, 1968. 


\section{Task 4.3: Implementation and Verification of the Cam Clay Model in}

NIKE-2D/ALE-3D Code By Ananda M. Wijesinghe and Kurt H. Sinz, LLNL

No activity scheduled for current reporting period.

\section{Task 4.4: Estimation of the Timing and Spatial Distribution of}

Rewetting By Ananda M. Wijesinghe and Kurt H. Sinz,, LLNL

No activity scheduled for current reporting period. 


\section{Summary, Conclusions \& Recommendations For Future Work}

\section{Summary}

This Interim Letter Progress Report describes the work performed in the first 8 months of the first year work-scope described in the Joint Work Statement attached as Appendix-A of this report. Initial studies have been conducted on several tasks. Work will continue on the remaining first year work-scope. Continuation of the Activities into the second year work-scope of the Joint Work Statement is proposed.

\section{ACTIVITY 1}

An initial evaluation of the effects of iron on the dissolution of borosilicate glass has been conducted in Task 1.1. Models and data applicable to this issue are discussed, along with their limitations. Recent experimental results have been included in this assessment. Further analysis must await additional experimental data and model evolution. No activity is reported on Task 1.2 at this time, however, the modeling methodology and data needs are being assessed.

\section{ACTIVITY 2}

The model and data framework for Activity 2 is discussed. As both the LLNL and JNC chemical modeling has evolved since the initial work planning, discussions will continue on focusing further database development to suit JNCs needs.

\section{ACTIVITY 3}

As planned in the Joint Work Statement, most of Activity 3 is deferred to the later part of the collaboration. Initial plans for the transient EBS analysis is discussed. Information from the draft $\mathrm{H} 12$ report and current experimental results from LLNL testing will be used to frame this future analysis.

\section{ACTIVITY 4}

\section{Progress}

We completed the basic requirements of sub-Task 4.1, and demonstrated that the ALE-3D code is capable of modeling clay extrusion into fractures, by successfully computing an uncoupled-process problem (Problem 1) and a coupled-process problem (Problem 2). In both problems, a single vertical fracture, 1-mm wide, was located at the center of the floor of a $0.5-\mathrm{m}$ wide $\mathrm{x} 0.5-\mathrm{m}$ tall rigid-walled clay-filled tunnel. In the first uncoupled-processes problem, the driving force for clay extrusion was a uniform pressure applied to the clay at the roof of the tunnel. In the second coupled-processes problem, the driving force was derived from the swelling of the clay when wetted by water that infiltrated into the clay from the tunnel roof. We treated the tunnel and the fracture as 
rigid and used estimated material properties for the clay. The ALE-3D code computed these two problems without any difficulty with explicit time stepping methodology by using mass scaling to allow the use of large time steps. It handled both the extreme distortion of the clay at the entry to the fracture and the movement of a wetting front into the clay. However, no independent verification of the accuracy of the computed results was made due to limitations in time and funds.

We also met the basic requirements of sub-Task 4.2 , by successfully computing a more realistic problem (Problem 3) of the downward migration of a steel overpack emplaced in a clay-filled tunnel. The downward settlement was due to elasto-plastic flow of clay around the overpack driven by the weight of the overpack, the stress increase due to clay swelling above the overpack due to water influx at the tunnel roof and the wetting induced softening of clay that supported the steel overpack. Frictional interfaces were used along the tunnel walls and the overpack surface. Displacement of the overpack varied from no settlement to some settlement as the yield strength was reduced over a practical range. The frictional shear stress along the sides of the overpack was a significant factor that retarded the downward movement of the overpack. As in the case of sub-Task 4.1, no independent verification of the accuracy of the computed results was made due to limitations in time and funds.

\section{Model Applicability}

We developed a rigorous theoretical model of the coupled processes that govern water transport within a porous elasto-plastically deforming clay material subject to swelling. By examining the approximations necessary to reduce this rigorous formulation to form of the governing equations solved in the ALE-3D code, we identified the assumptions that must be made to utilize the existing ALE-3D code to compute the problems of interest to this Task. The most important of these approximations are:

1. The clay behaves as a porous deformable material salisfying an effective stress law for partitioning the total stress between the solid and fluid phases,

2. The constitutive behavior of effective solid stress for clay satisfies an associated Drucker-Prager type frictional elasto-plastic flow model extended to incorporate isotropic swelling of clay and water saturation dependent plasticity parameters,

3. The transport of water within the clay under unsaturated conditions can be described in terms of relative permeability and capillary pressure relationships,

4. The pressure of the air phase within the pores remains essentially constant.

Under these assumptions, the governing equations were reduced to two coupled field equations. The first equation governs the deformation of the clay and has the form of a thermo-elasto-plasto-dynamic equation when water saturation is identified with temperature. The second equation has the form of a heat diffusion equation when water saturation is again replaced by temperature. Consequently, the coupled heat conduction and thermo-elasto-plasto-dynamic deformation modelling capabilities of ALE-3D can be adapted to compute the problems of coupled hydro-elasto-plastic deformation and water 
transport of interest to this Task. We believe all of these approximations are reasonable and valid in this application.

However, in computing Problems 2 and 3 we were constrained by deficiencies in the ALE-3D code. We could not use the full extended Drucker Prager model for two reasons: 1) some of the terms arising from the dependence of the plastic flow rule on changes in water saturation (as identified in Chapter 2) were not represented in the ALE3D code, and 2) the dependence of frictional Drucker-Prager model parameter on water saturation was not available in the ALE-3D code. For this reason, we used a simple only water saturation dependent yield stress model (i.e., water saturation dependent cohesion with the frictional parameter equal to zero). However, these features can be incorporated in the ALE-3D code with some effort in the future.

Another feature, not within the scope of AI.E-3D code design, is that water transport has to be modelled approximately via a non-linear diffusion equation. Although it was not a problem in the present application, it would be more general and accurate to avoid the assumption of constant air pressure, and to independently solve the equations for water and air transport for one fluid phase pressure and the water saturation.

\section{Computational Methodology}

The problems addressed here are essentially quasi-static processes that are slow compared to the time of transit of an elastic wave through the clay, and therefore, usually require calculation-intensive implicit time stepping strategies to enable large time steps to be taken. The ALE-3D code, on the otherhand, is designed to solve dynamic problems over time periods that are comparable to the wave transit time, and therefore utilizes relatively simple but accurate explicit time stepping strategies based on integrating the inertial acceleration term in time. To enable the ALE-3D code to compute our quasi-static problem with large time steps and explicit time stepping, we used the ALE-3D code option of scaling the mass and time in the inertia term. In this way, we were able to compute all three problems with a reasonable amount of computational effort.

Although the ALE-3D code had certain deficiencies in its constitutive models for our application, the great advantage it offered in coping with large deformations and moving material interfaces without manual intervention to regenerate and/or refine the computational grid, was invaluable. This feature yielded very large savings in labor and computational time and enabled these preliminary calculations to be completed within a relatively short time period.

\section{Conclusions \& Recommendations For Future Work}

\section{ACTIVITY 1}


Further evaluation of iron effects on glass dissolution will focus on new data and model evolution. Calculations of overpack-bentonite interactions will begin in the remainder of the first year work-scope, and are proposed for continuation in the secondyear.

\section{AC'IIVI'TY 2}

Further interaction is underway between JNC and LLNL to re-focus the geochemical database development task. Analysis of solubility controls on selenium are proposed for the second-year workscope in the Joint Work Statement

\section{ACTIVITY 3}

During the remainder of the first-year work, information from the draft JNC H12 report will be used to develop the conditions for transient EBS analysis. Performance of this analysis is proposed for the second-year workscope of the Joint Work Statement.

\section{ACTivity 4}

\section{Model Formulation \& Code Enhancements}

We recommend continued use of the ALE-3D code for the applications in this Task and the implementation of certain code enhancements to adapt and improve the constitutive models incorporated in the code in Task 4.3.

First, we propose that the full extended Drucker-Prager frictional elasto-plastic model with allowance for clay swelling and water saturation dependent parameters, that is presented in this report, be implemented in the code and tested against other codes and available experimental data.

Second, we propose that the work be extended to include an evaluation of both the Cam Clay [8] and the Sandler-DiMaggio Moving Cap [7] elasto-plastic model types and the better of these two types of frictional elasto-plastic models be incorporated in the ALE-3D code as an alternative to the Extended Drucker-Prager model presented in this report. Without further investigation and studies based on measured material property data on clays, it is not possible to predict which of these different types of elasto-plastic models would be better for use in the current application.

\section{Computations}

We have demonstrated the ability of the ALE-3D code to handle clay extrusion into fractures, the principal issue of interest to the JNC. However, although this work has demonstrated that the ALE-3D code can handle the extremely large material distortion associated with clay extrusion into fractures, and can compute overpack migration problems involving coupled processes of plastic deformation of clay and water infiltration, the calculations were perfomed with roughly estimated clay material 
properties. Also, the calculations assumed a rigid rock mass and non-deformable fractures, thus possibly underestimates the impact of clay extrusion into fractures. Therefore, the results must be verified using more appropriate material property cxperimental data and checked against other calculations or direct measurements of the extent of overpack displacement and clay extrusion into fractures. This could not be done due to limitations in the available funds, project time and material property data. No conclusion on the significance of impact of clay extrusion into fractures on overpack migration can be made at this time on the basis of these limited calculations; that must await the results of the more detailed parametric study that will be undertaken in subTasks 4.3 and 4.4 .

Upon completion of the above two code enhancements, the codes should be verified against other codes, published solutions and/or available experimental data. Then Tasks 4.1 and 4.2 should be repeated and the results compared. The calculations should be extended to include deformation of the tunnel and the fractures, and if feasible, adopt more realistic tunnel/emplacement/overpack geometries and material properties relevant to, and derived from, JNC's on-going applications/experimental program.

Modeling work under Task 4.4 geared to estimation of the timing, the spatial distribution of rewetting, and overpack migration in the tunnel should be undertaken as originally proposed for this portion of this Task.

\section{Supporting Experiments}

In addition to bench-scale experiments performed to determine the material properties of the backfill materials, it would be useful to undertake a field-scale experiment to verify and demonstrate that the predictions made with the analysis codes are in fact realistic under as-emplaced repository conditions. For this purpose, we recommend that a field scale experiment be designed and executed in a fractured rock mass, and the mesurements be compared against both pre-test predictive analyses and post-test performance analyses. 


\section{Appendix A: Joint Work Statement}




\title{
JOINT WORK STATEMENT
}

\author{
FOR THE ANNEX TO THE AGREEMENT BETWEEN \\ POWER REACTOR AND NUCLEAR FUEL DEVELOPMENT CORPORATION \\ OF JAPAN (PNC) AND THE UNITED STATES DEPARTMENT OF ENERGY (DOE) \\ ON THE COOPERATION IN THE AREA OF RADIOACTIVE WASTE MANAGEMENT
}

\section{THE STUDY FOR THE NEAR-FIELD PERORMANCE ANALYSES ON THE LONG-TERM BEHAVIOR OF THE ENGINEERED BARRIERS}

\section{INTRODUCTION}

Under this Annex, a research program on the near-field performance assessment related to the geological disposal of radioactive waste will be carried out at the Lawrence Livermore National Laboratory (LLNL) in close collaboration with the Power Reactor and Nuclear Fuel Development Corporation of Japan (PNC). This program will focus on activities that provide direct support for PNC's near-term and long-term needs that will, in turn, utilize and further strengthen U.S. capabilities for radioactive waste management.

The work scope for two years will be designed based on the PNC's priorities for its second progress report (the H12 report) of research and development for high-level radioactive waste disposal and on the interest and capabilities of the LLNL. The work will focus on the chemical modeling for the near-field environment and long-term mechanical modeling of engineered barrier system as it evolves. Certain activities in this program will provide for a final iteration of analyses to provide additional technical basis prior to the year 2000 as determined in discussions with the PNC's technical coordinator. The work for two years will include the following activities;

Activity 1: Chemical Modeling of EBS Materials Interactions.

Task 1.1 Chemical Modeling of Iron Effects on Borosilicate Glass Durability

Task 1.2 Changes in Overpack and Bentonite Properties Due to Metal, Bentonite and Water Interactions

Activity 2: Thermodynamic Database Validation and Comparison.

Task 2.1 Set up EQ3/6 to Run with the Pitzer-based PNC Thermodynamic Data Base

Task 2.2 Provide Expert Consultation on the Thermodynamic Data Base

Task 2.3 Provide Analysis of Likely Solubility Controls on Selenium

Activity 3: Engineered Barrier Performance Assessment of the Unsaturated, Oxidizing Transient.

Task 3.1 Apply YMIM to PNC Transient EBS Performance

Task 3.2 Demonstrate Methods for Modeling the Return to Reducing Conditions

Task 3.3 Evaluate the Potential for Stress Corrosion Cracking in PNC Waste Packages

Activity 4: Coupled Displacement and Degradation Analysis of Carbon Steel Overpack Embedded in Bentonite.

Task 4.1 Demonstration of NIKE-2D/ALE-3D Mesh Adaptation Capability

Task 4.2 Demonstration of NIKE-2D/ALE-3D Code Capability to Compute Realistic Repository Problems

Task 4.3 Implementation and Verification of the Cam Clay Model in NIKE-2D/ALE-3D Code

Task 4.4 Estimation of the Timing and Spatial Distribution of Rewetting

Much of the work outlined below will be accomplished through work at both PNC and LLNL, with communications through written reports, workshops, and/or short technical visits, personnel exchange through the PNC international fellowship program, and extended technical visits and exchanges by PNC and LLNL scientists to participate in experiments and/or model studies at LLNL and PNC. 
Results of this collaborative program will be documented in annual progress reports: an interim progress letter report to be completed March 30,1999 and a final formal report in early 2000 coordinated to support the deliverable $\mathrm{H} 12$ report. The content of these reports is summarized at the end of this Joint Work Statement.

\section{DESCRIPTSION OF TASKS}

\section{ACTIVITY 1: Chemical Modeling of EBS Materials Interactions}

There are two tasks for this activity:

- Chemical Modeling of Iron Effects on Borosilicate Glass Durability Provide

- Changes in Container and Bentonite Properties Due to Metal, Bentonite and Water Interactions

\section{Task 1.1: Chemical Modeling of Iron Effects on Borosilicate Glass Durability}

Goal:

Demonstrate methods of modeling the effect of iron corrosion products on the long-term durability of a borosilicate glass waste form.

\section{Scope:}

A matrix of model calculations of glass corrosion rates for relevant repository conditions with and without iron present will be carried out. This matrix will also account for varying hydrologic scenarios, water chemistries, and the effects of backfill. Conservative assumptions will be made when necessary. The product will be qualitative information on beneficial vs. harmful effects of iron on glass wasteform performance and relative magnitudes of these effects. The model used for the calculations should be reviewed against the existing literature. Optimum experiments for model validation will also be identified.

In order to perform these modeling calculations, thermodynamic data and sorption coefficients for appropriate aqueous and solid iron species must be incorporated into the database accessed by the model. Most of the available thermodynamic data for iron species are already included in the existing database. However, data for sorption coefficients (surface complexation constants) need to be collected, evaluated, and entered into the data file. The modeling code of choice for this project (GWB) currently provides for sorption, ion exchange, and dissolution/precipitation of metastable species in redox disequilibrium, so that no code development will be necessary. Some thermochemical data may need to be estimated using correlation techniques based on analogous species and reactions for which data are available.

\section{Reporting:}

The interim LLNL report to PNC will include the initial activities of this task including data acquired or developed, criteria used to select data, data sources, a description of the analyses to be performed and a preliminary description of the database selected. and present preliminary results of the modeling studies. The final LLNL report to PNC will document the completed analysis results for use within the technical basis for the PNC $\mathrm{H} 12$ deliverable. As resources and priorities dictatate, it may provide qualitative information on iron effects on glass corrosion rates for a matrix of repository scenarios; identify outstanding data needs; and identify optimum model validation experiments.

\section{Task 1.2 - Changes in Overpack and Bentonite Properties Due to Metal, Bentonite and Water Interactions}

\section{Goal:}

Determine what interactions may take place between bentonite and bentonite-sand mixture buffer and a waste container, and how those interactions will change with time. This work will produce quantitative models that describe the changes in water chemistry, container surface mineralogy and bentonite properties over the lifetime of the engineered barrier system (EBS). 


\section{Scope:}

Bentonite based buffer and metal overpacks are not in chemical equilibrium with each other. The presence of water, either as vapor or liquid, will lead to chemical reactions between these materials that will change the swelling and sorptive properties of the buffer, and will modify the overpack. These processes can significantly influence overpack lifetime, backfill behavior, and contaminant transport.

Using available data on reaction rates of the initial overpack materials and buffer mineralogy, and recently developed computer models of chemical interactions in advecting, transporting systems, the rates of mineralogical changes in the backfill will be documented. Also addressed will be the changes that occur at the overpack-buffer interface, including quantitative descriptions of the changes in overpack surface mineralogy, and changes in local porosity, as a function of time. These recent developments make it possible to extend previous studies, which have examined parts of this complex system, to much more realistic conditions. Most significantly for these studies, the rates at which the chemical reactions occur can be accurately included, taking into account the influences of evolving gas and water chemistry, and changes in mineralogy. This study will produce detailed descriptions of the interactions between buffer and waste container within a PNC-designed EBS.

\section{Reporting:}

A chapter of the interim LLNL report to PNC will describe the model methodology and database for the analysis and will include options for the final analysis. The final LLNL report will include results of this analysis.

\section{ACTIVITY 2: Thermodynamic Database Validation and Comparison}

\section{Goal:}

To provide a coordinated effort combining computer modeling and laboratory experiments, in order to build confidence in the thermodynamic database used by PNC for predicting potential radionuclide release and migration from an underground repository.

There are three tasks for this activity:

- Set up EQ3/6 to Run with the Pitzer-based PNC Thermodynamic Data Base

- Provide Expert Consultation on the Thermodynamic Data Base

- Provide Analysis of Likely Solubility Controls on Selenium

\section{Task 2.1. - Set up EQ3/6 to Run with the Pitzer-based PNC Thermodynamic Data Base}

\section{Scope:}

LLNL will modify EQ3/6 to allow it to make calculations using the PNC thermodynamic data base. This will allow PNC investigators to make geochemical calculations using EQ3/6 and a PNC data file, as well as with other $\mathrm{EQ} 3 / 6$ data files.

PNC will provide LLNL with an ASCII copy of the PNC data base. LLNL will incorporate this into the GEMBOCHS relational data base, which is the source of all LLNL EQ3/6 data files. GEMBOCHS is capable of distinguishing among alternate data sets, so all the PNC data can be segregated within this relational data base. LLNL will then use GEMBOCHS to generate a "PNC" data file, analogous to various other EQ3/6 data files (e.g., "COM," "SUP," "NEA"). LLNL believes that this is the most cost-effective approach to generating a PNC data file for EQ3/6. Another advantage of using GEMBOCHS to manage the PNC data base is that this will facilitate any future changes to this data base, including replacing PNC data with other data in GEMBOCHS or adding such data to the PNC data. LLNL will provide PNC with a copy of the modified EQ3/6. LLNL may distribute the PNC data file to others unless PNC specifically objects.

\section{Reporting:}

The interim LLNL report to PNC will discuss the goals and methodology for the database development and report on database transfer status. The final LLNL report will include the modified EQ3/6 software package including a data file containing the PNC data base, code documentation, and sample problems. Unless a UNIX format is requested by PNC, the package will be in PC (Pentium) format. The final LLNL report may also include additional analysis and documentation as permitted by schedule and resources to support the H12 deliverable. 


\section{Task 2.2. - Provide Expert Consultation on the Thermodynamic Data Base}

\section{Scope:}

LLNL will provide expert consultation on the thermodynamic data base. This includes consultation with PNC's existing expert panel and examination of the Pitzer-based modeling work. The expert consultation will assist PNC in developing and justifying the PNC thermodynamic data base.

\section{Reporting:}

A report of this consultation will be included in each LLNL progress report to PNC, summarizing analysis and recommendations. Documentation will be included as appropriate in the final LLNL report to support the H12 deliverable analysis.

\section{Task 2.3: - Provide Analysis of Solubility Controls on Selenium}

\section{Scope:}

Selenium is a major concern in the early stages of PNC waste form leaching. The concentration of dissolved selenium may be limited by the formation of $\mathrm{Se}_{(\mathrm{c})}$ and $\mathrm{FeSe}_{2(\mathrm{c})}$, or by substitution of $\mathrm{Se}$ for $\mathrm{S}$ in the corresponding $S$ phases. LLNL will analyze experimental solubility data to be provided by PNC to see if it can be modeled assuming thermodynamic equilibrium. LLNL will provide a model or models for solubility controls on Selenium with report of analysis of the experimental data and recommendations for future work.

\section{Reporting:}

A chapter of the final LLNL report will contain LLNL's analysis of PNC's experimental data on Se solubility. This report will contain a model explaining these data if such a model is found feasible. The report will make recommendations for future experimental or theoretical work as appears desirable or necessary.

\section{ACTIVITY 3: Engineered Barrier Performance Assessment}

\section{Goal:}

To demonstrate performance modeling of the engineered barrier system during the operational time and during the transition back to saturated and reducing conditions.

The PNC repository design has a transient period of unsaturated oxidizing conditions with elevated EBS temperatures. This period begins during repository operations and ends at some time after closure when water resaturation occurs and the operational oxygen is consumed. Models developed for the US repository in unsaturated oxidizing conditions may prove useful in modeling PNC repository EBS performance during this period.

There are three tasks for this activity:

- Apply YMIM to PNC transient EBS performance

- Demonstrate methods for modeling the return to reducing conditions

- Evaluate the potential for stress corrosion cracking in PNC waste packages

\section{Task 3.1 - Apply YMIM to PNC Transient EBS Performance}

\section{Scope:}

LLNL will use the YMIM Near-Field Environment and Engineered Barrier System model to assess waste package corrosion during the unsaturated oxidizing temperature transient in the PNC repository design. Code input will be modified to approximate PNC repository design and conditions and example calculations performed. The strengths and weaknesses of this model for the PNC transient analysis will be discussed and recommendations for more detailed analyses made where appropriate. Output will include examples of calculated container corrosion depth from both general and localized corrosion processes.

\section{Reporting:}

Most of this activity will be performed in the second year of the study. Discussion with PNC will determine the analyses to be performed to support deliverable H12 and reported in the LLNL final report. 


\section{Task 3.2 - Demonstrate Methods for Modeling the Return to Reducing Conditions}

\section{Scope:}

Changes in the redox state of the waste package environment can be expected to occur as backfill material heats up. These changes will be due to a range of effects, including interaction of the waste package with dissolved gases in the fluid surrounding the containers, interaction of backfill, rock, water and waste packages as temperatures evolve, and the response of the excavated repository to perturbations caused by engineering and operation of the repository.

In previous studies, LLNL has conducted a wide range of simulations for saturated and unsaturated conditions, and supporting experiments, to bound the evolution of the redox state in the environment surrounding waste packages for the US repository program. Emphasis in these studies has been on establishing the rate at which changes would occur, taking into account estimated quantities of materials emplaced within the potential repository. We propose to demonstrate similar bounding studies for the PNC program.

The work would be conducted by modeling the rate at which changes occur in the redox state, using reactive transport simulators, along with estimates of the amount and types of materials placed in the PNC repository, and estimates of the evolution of the thermohydrologic regime in the vicinity of waste packages. Demonstration simulations would be conducted that would bound the types of change that might occur on the scale of $\mathrm{mm}$ to $\mathrm{cm}$, and on the scale of a few meters. This will allow estimation of the rates of change in selected portions of the backfill-container system, to allow determination of the redox environment waste package surfaces may experience, as a function of time.

\section{Reporting:}

The methodology for this analysis will be presented in the interim LLNL report to PNC with results summarized in the final report. The detailed analyses and documentation included in the final report will be determined in discussions with PNC staff.

\section{Task 3.3 - Evaluate the Potential for Stress Corrosion Cracking in PNC Waste Packages}

\section{Scope:}

Under the conditions of the PNC repository, it is expected that general corrosion of the waste package will be relatively slow. Under such conditions, there is concern regarding stress corrosion cracking of the closure welds. LLNL is testing welded and unwelded carbon steel and low alloy steel for stress corrosion cracking, and localized and general corrosion under oxidizing aqueous and saturated vapor conditions. U-bend specimens are employed for detection of susceptibility to stress corrosion cracking. The aqueous solutions have both low (2000 ppm) and high $(120,000 \mathrm{ppm})$ ionic content, and are slightly basic (pH 8-9). 'Test temperatures are 60 and $900 \mathrm{C}$. The testing is ongoing, continuous, and anticipated to last at least 5 years. The information from this testing will be fed into models that will predict the susceptibility of carbon steel to stress corrosion cracking.

\section{Reporting:}

This work will be done late in the schedule to take advantage of a long a testing period as possible. The plan for this analysis will be included in the interim LLNL report. The results will be included in the final report.

\section{ACTIVITY 4: Coupled Displacement and Degradation Analysis of a Carbon Steel Overpack Embedded in Bentonite}

\section{Goal:}

To test and demonstrate capabilities for modeling the displacement of waste packages in clay and clay-sand mixture driven by symmetric and asymmetric processes.

There are four tasks for this activity:

- Demonstration of NIKE-2D/ALE-3D Mesh Adaptation Capability

- Demonstration of NIKE-2D/ALE-3D Code Capability to Compute Realistic Repository Problems

- Implementation and Verification of the Cam Clay Model in NIKE-2D/ALE-3D Code

- Estimation of the Timing and Spatial Distribution of Rewetting 


\section{Task 4.1 - Demonstration of NIKE-2D/ALE-3D Mesh Adaptation Capability}

\section{Scope:}

Demonstrate the grid adaptation and remeshing capabilities of the NIKE-2D (or ALE-3D) code by computing an axisymmetric problem of extrusion of bentonite into fractures due to homogeneous swelling of the bentonite upon wetting. The ALE-3D code run in the $2 \mathrm{D}$ mode may prove to be more suitable than the NIKE-2D code for modeling extrusion into narrow fractures. Utilize the existing elasto-plastic constitutive models and the thermal expansion modeling capabilities of the codes to represent bentonite swelling.

\section{Reporting:}

This work will be done in the first year of the study and reported in the interim LLNL report to PNC.

\section{Task 4.2 - Demonstration of NIKE-2D/ALE-3D Code Capability to Compute Realistic Repository Problems}

\section{Scope:}

Demonstrate the capability of the NIKE-2D (or ALE-3D) code to compute a more realistic repository problem by computing the migration of an overpack that is non-symmetrically emplaced in a vertical borehole. Assume homogeneous swelling and adopt existing elasto-plastic models and allow for extrusion of bentonite into fractures, as in Task 4.1.

\section{Reporting:}

This work will be done in the first year of the study and reported in the interim LLNL report to PNC.

\section{Task 4.3: - Implementation and Verification of the Cam Clay Model in the NIKE-2D/ALE-3D Code}

\section{Scope:}

If Tasks 4.1 and 4.2 are successfully completed, embed the Cam Clay constitutive model in the NIKE-2D/ALE3D code, verify the code, and repeat Tasks 4.1 and 4.2. The applicability of existing elasto-plastic model and Cam Clay model to bentonite-sand mixture system will also be discussed.

\section{Reporting:}

The plan for this work will be presented in the interim LLNL report to PNC. Documentation of the results will be included in the final LLNL report. This will describe the problems computed, the difficulties encountered and an assessment of the capabilities of the NIKE-2D/ALE-3D and flow and transport codes required to achieve the objectives of each of these Tasks. Discussion with PNC staff will prioritize the analysis and documentation needed in the final report to support the $\mathrm{H} 12$ deliverable.

\section{Task 4.4 - Estimation of the Timing and Spatial Distribution of Rewetting}

Scope:

Model the rewetting process and estimate the timing and distribution of groundwater in the repository near-field, and within the emplacement borehole, using a suitable tinite element code for unsaturated/ saturated groundwater flow. Utilize the finite element grid generated from the NIKE-2D/ALE-3D code in Task 4.2 above. Evaluate the feasibility of closely coupling the stress-deformation and rewetting processes utilizing these two separate codes.

\section{Reporting:}

The methodology for this work will be presented in the interim LLNL report to PNC. The final LLNL report will include documentation of the entire scope of work. This report will provide an assessment of the overall feasibility of utilizing the NIKE-2D/ALE-3D code and the finite-element based flow and transport code in a coupled manner to support detailed analyses of this class of problems in the future. 


\section{Report Content Summary}

Interim Progress Letter Report (March 30, 1999)

Activity 1: Chemical Modeling of EBS Materials Interactions.

Model methodology and database development for the analysis of both glass degradation and bentonite interactions, including preliminary results.

Activity 2: $\quad$ Thermodynamic Database Validation and Comparison.

Methodology, goals and database transfer status.

Activity 3: $\quad$ Engineered Barrier Performance Assessment of the Unsaturated, Oxidixing Transient.

Methodology for model of return to reducing conditions.

Activity 4: $\quad$ Coupled Displacement and Degradation Analysis of a Carbon Steel Overpack Embedded in Bentonite.

Deformation code grid adaptation and repository application results. Plan for Cam Clay model incorporation.

Final Report (date to be determined to support H12)

Activity 1: Chemical Modeling of EBS Materials Interactions.

Document the completed analysis results for use within the technical basis for the PNC H12 deliverable.

Activity 2: $\quad$ Thermodynamic Database Validation and Comparison.

Delivery of modified EQ3/6 software package including PNC data base. Additional analysis and documentation as required to support the H12 deliverable. Analysis of Selenium solubility data.

Activity 3: $\quad$ Engineered Barrier Performance Assessment of the Unsaturated, Oxidizing Transient.

Analysis results for both return to reducing conditions and EBS performance. Assessment of stress corrosion cracking potential.

Activity 4: $\quad$ Coupled Displacement and Degradation Analysis of a Carbon Steel Overpack Embedded in Bentonite.

Final analysis of perturbed flow and displacements and rewetting analysis. 